\title{
Synchronization and Stability in Dynamical Models of Power Supply Networks
}

\author{
Dissertation \\ zur Erlangung des mathematisch-naturwissenschaftlichen Doktorgrades \\ "Doktor rerum naturalium" \\ der Georg-August-Universität Göttingen
}

vorgelegt von

Martin Rohden

aus Uelzen

Göttingen 2013 


\section{Mitglieder des Betreuungsausschusses:}

Prof. Dr. Marc Timme (Referent)

Network Dynamics

Max Planck Institute for Dynamics and Self-Organization

Prof. Dr. Annette Zippelius

Institute for Theoretical Physics

Georg-August-Universität Göttingen

Prof. Dr. Rainer Kree (Referent)

Institute for Theoretical Physics

Georg-August-Universität Göttingen

\section{Weitere Mitglieder der Prüfungskommision:}

Prof. Dr. Stephan Herminghaus

Dynamics of Complex Fluids

Max Planck Institute for Dynamics and Self-Organization

Prof. Dr. Florentin Wörgötter

Third Institute of Physics - Biophysics

Georg-August-Universität Göttingen

Prof. Dr. Ulrich Parlitz

Biomedical Physics

Max Planck Institute for Dynamics and Self-Organization

Tag der mündlichen Prüfung: 14.01.2014 
I comfirm that I have written this thesis independently and with no other sources and aids than quoted.

Göttingen, 



\section{Contents}

\begin{tabular}{lll}
\hline 1 & Kurzfassung & 7
\end{tabular}

$\begin{array}{lll}2 & \text { Abstract } & 9\end{array}$

3 Introduction 11

$\begin{array}{|ll|}4 \text { An oscillator model for power grid operation } & 17\end{array}$

4.1 Overview . . . . . . . . . . . . . . . . . . . . . . . . . . . . . . 17

4.2 Oscillator model . . . . . . . . . . . . . . . . . . . . . . . . . . . . . 18

4.2 .1 Synchronous machines . . . . . . . . . . . . . . . . . . . . 18

4.2 .2 Alternating current $\ldots \ldots \ldots \ldots \ldots$

4.2 .3 Power transmission in alternating current circuits . . . . . . . . . 21

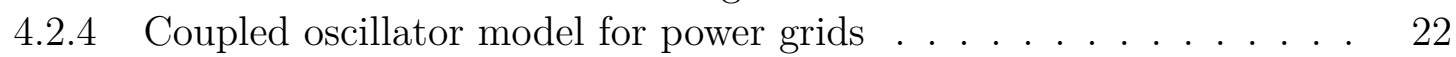

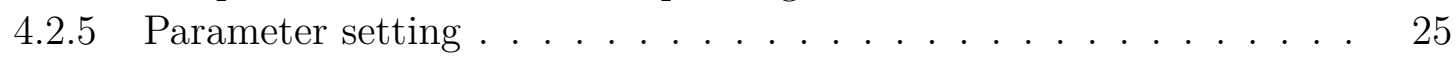

$4.2 .6 \quad$ Comparison with Kuramoto model $\ldots \ldots \ldots \ldots$

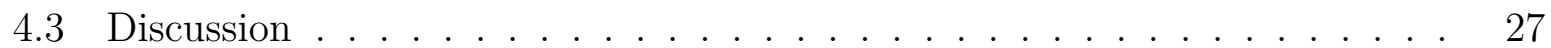

5 Dynamics \& self-organization of an elementary model 29

5.1 Effective equations of motion $\ldots \ldots \ldots \ldots \ldots$

5.2 Bifurcation structure $\ldots \ldots \ldots \ldots \ldots \ldots$

5.3 Global stability properties $\ldots \ldots \ldots \ldots$. . . . . . . . . . . . . . 34

5.4 Equivalence to the dynamics in a tilted washboard potential $\ldots \ldots . . .37$

5.5 Imbalanced power production and consumption $\ldots \ldots \ldots \ldots$

5.6 Self-organized adaptation $\ldots \ldots \ldots \ldots . \ldots \ldots$

$5.7 \quad$ Discussion $\ldots \ldots \ldots \ldots \ldots$. . . . . . . . . . . . . . . . . . . . . . . . . . . . 42

6 Decentralized power generation in future power grids 45

6.1 Modeling the decentralization process . . . . . . . . . . . . . 46

6.1.1 Modeling the decentralization process of random network ensembles 46

6.1 .2 Modeling the decentralization process of the British grid $\ldots \ldots .48$

6.2 The synchronization transition $\ldots \ldots \ldots \ldots \ldots$

6.2 .1 Synchronization transition of motif networks . . . . . . . . . . 49

$6.2 .2 \quad$ Synchronization transition of random model networks . . . . . . . . 51

6.2 .3 Synchronization transition of the British grid . . . . . . . . . . . . 54

6.3 Synchronization time $\ldots \ldots \ldots \ldots \ldots \ldots \ldots \ldots \ldots \ldots$ 


\section{Contents}

6.4 Robustness against dynamic perturbations . . . . . . . . . . . . . . . . . 59

6.4.1 Robustness of random network ensembles against dynamic pertur-

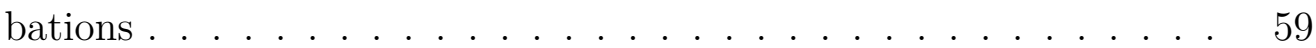

6.4 .2 Robustness of the British power grid against dynamic perturbations 61

6.5 Stability against structural damages . . . . . . . . . . . . . . . 62

6.6 Summary and discussion . . . . . . . . . . . . . . . . . . . . . . . . . . . . 64

$\begin{array}{lll}7 & \text { Power outages and critical transmission lines } & 65\end{array}$

7.1 Transmission line failures . . . . . . . . . . . . . . . . . . . . . . . . . . 66

7.2 Simulation methods . . . . . . . . . . . . . . . . . . . . . 67

7.3 Breakdowns of transmission lines in periods of low loads . . . . . . . . . . 67

7.4 Breakdowns of transmission lines in periods of high loads . . . . . . . . . . 70

$7.4 .1 \quad$ Stability or large scale outage? . . . . . . . . . . . . . . . . 70

7.4 .2 Redundancy in power grids . . . . . . . . . . . . . . . . . . . 71

7.4 .3 Performance of the statistical testing methods . . . . . . . . . . . . 74

7.5 Response to small local changes . . . . . . . . . . . . . . . . . 77

8.6 Discussion . . . . . . . . . . . . . . . . . . . . . . . . . . . . 81

\begin{tabular}{lll}
\hline 8 & Conclusions & 83
\end{tabular} 


\section{Kurzfassung}

In der Stromversorgung vollzieht sich seit etwa zwanzig Jahren ein grundlegender Wandel von konventioneller Stromerzeugung durch hauptsächlich Kohle- und Atomkraftwerke hin zu erneuerbaren Stromerzeugung durch hauptsächlich Windkraft- und Solaranlagen. Die Hauptunterschiede zwischen diesen beiden Formen der Erzeugung liegt in der geringeren Leistung, die eneuerbare Erzeuger typischerweise gegenüber konventionellen Erzeugern produzieren und in dem vermehrten Auftreten von Fluktuationen in deren Leistungserzeugung. Aufgrund dieser fundamentalen Unterschiede zwischen diesen beiden Formen der Stromerzeugung stellt dieser Wandel das stabile Funktionieren des Stromnetzes vor gros̈se Herausforderungen.

In dieser Arbeit werden anhand eines einfachen Modells für Stromnetze verschiedene Fragestellungen die Stabilität des Stromnetzes betreffend untersucht. Im ersten Teil der Arbeit wird das Modell im Detail vorgestellt. Anschlies̈send wird das Modell für das einfachst möglichste Stromnetz untersucht, hier bestehend aus einem Erzeuger und einem Verbraucher. In diesem einfachen Fall lassen sich die mathematischen Gleichungen des Modells analytisch lösen, was Einsichten in die Eigenschaften des Modells erlaubt. Diese Eigenschaften sind auch bei grös̈seren Netzen vorzufinden. Es wird gezeigt, dass das Modell die wichtigsten Eigenschaften des realen Netzes erfasst, an erster Stelle dass die Entwicklung der Dynamik des Stromnetzes sowohl hin zu einem stabilen Zustand, als auch zu einem instabilen Zustand hin möglich ist, abhängig von dem aktuellen Zustand des Netzes.

Im zweiten Teil der Arbeit wird das Phänomen der Dezentralisierung untersucht. Da Erzeuger, die auf erneuerbaren Energieträgern basieren, typischerweise weniger Leistung produzieren koennen als konventionelle Kraftwerke, müssen, um einzelne konventionelle Kraftwerke zu ersetzen, mehrere erneuerbare Erzeuger neu an das bestehende Stromnetz angeschlossen werden. Dies führt zu der sogenannten Dezentralisierung, womit gemeint ist, dass die neu angeschlossen Kraftwerke oftmals weit von der Masse der Verbraucher, zum Beispiel gros̈ser Städte, entfernt sind. Dies hat verschiedene Konsequenzen auf die Stabilität des Stromnetzes, welche in diesem Kapitel im Einzelnen untersucht werden. Zusammenfassend lässt sich sagen, dass die Robustheit des Netzes gegen gros̈se Störungen fuer dezentrale Netze geringer ist als für zentrale, während die strukturelle Stabilität gegen einzelne Leitungsausfälle zunimmt.

Im letzten Teil der Arbeit wird die strukturelle Stablität des Stromnetzes genauer untersucht. Es werden neuartige Verfahren entwickelt, um Vorhersagen zu können, welche Leitungen, falls sie ausfallen sollten, einen systemweiten Stromausfall nach sich ziehen und 


\section{Kurzfassung}

welche nicht. Es wird gezeigt, dass die Leistung, die eine Leitung transportiert, kein ausreichendes Kriterium ist, um präzise Vorhersagen für das Verhalten des gesamten Netzes im Falle des Ausfalls einer Leitung zu treffen. Abschliessend werden im Detail neue Kriterien entwickelt, die sich für Vorhersagen als geeignet herausstellen. 


\section{Abstract}

We are currently witnessing a change of electrical power supply from conventional power generation to power generation based on renewable energy sources such as wind and solar power. The main differences between these two ways of power generation are that power generation based on renewables produces typically less and more fluctuating power output. The changes in power production therefore pose a problem for the stability of power grids.

In this thesis we analyze different effects of these changes for the stability of power grids. In the first part we introduce in detail the power grid model we are using. We analyze the model for the simplest possible system, a system consisting of one generator and one consumer. For this system an analytical analysis of the model is possible. We demonstrate that the model contains the most important features of real-world power grids and is such a useful tool for investigations of larger networks. The most important feature is that there exists both a stable and an unstable state and the dynamics depend on the initial conditions, i.e., the current state of the network.

In the second part of the thesis we investigate the process of decentralization of power generation. This process is ongoing due to the replacement of conventional power sources with renewables. As renewable power sources are typically far away from consumers the replacements process ends up with a decentralized power grid. Our main results are that dynamical stability, i.e., stability against large scale power perturbations, is decreased for decentralized power grids compared with centralized ones. On the other hand, structural stability, i.e., stability against single transmission line failures, is increased for decentralized grids.

In the last part we analyze structural stability in more detail. We develop a novel criterion to predict which transmission lines can induce large scale power outages if they fail and which not. We demonstrate that the load of a transmission line alone is not a good predictor for this behavior. 



\section{Introduction}

A reliable supply of electric power fundamentally underlies the function of most of our technical infrastructure and affects all aspects of daily life [1, 2]. It is thus an essential task to maintain a stable operation of the power grid and to minimize the risk of power outages. The supply of electric power consists of power generation, power distribution and trade. It is based on a complex technical infrastructure and a regulatory framework. These conditions are subject to constant changes.

Today we are witnessing a time of rapid changes of power generation. These changes are mainly driven by concerns for environment and climate. It is nowadays scientific consensus that one of the main reasons for global warming is the human-caused emission of greenhouse gases such as carbon dioxide and methane [3]. To reduce global warming, Germany has committed itself to reduce the production of greenhouse gases until 2050 by $80 \%$ to $95 \%$ compared to the production level of 1990 [4]. Among the main producers of carbon dioxide are conventional coal-fired power plants [3], which have been a major power source since the early years of power grids and in 1990 still produced more than $60 \%$ of the total power in Germany [5]. As carbon dioxide is one of the main greenhouse gases causing global warming, the reduction of power generation by coal-fired power plants is one of the main concepts to reduce the output of carbon dioxide and thus to meet the reduction commitment [4]. Therefore, the amount of power generation by renewable energy sources has constantly been increasing in Germany for the last 20 years. In 1990 the fraction of renewable energy sources on the total power production was only $3.4 \%$, in 2000 it was $6.2 \%$ and in 2012 it already amounted to 23.5\% 6]. According to the so-called "Energiewende" [4], planned by the German government, the goal is to increase this fraction to $35 \%$ in 2020 and even to $80 \%$ in 2050 [7]. Renewable energy sources are mainly based on wind, solar and hydroelectric power and produce a lot less greenhouse gases than coal power plants. Consequently, the amount of greenhouse gases is today already reduced by $25 \%$ compared to the level of 1990 [4]. Renewable energy sources are therefore the desired form of energy production in the future.

The increase of the inclusion of renewable energy sources into the power grid gained momentum in Europe when in 1996 the European Union issued a directive to liberalize energy markets (see [8]). In Germany the inclusion of renewable energy sources into the power grid gained momentum after the enacting of the "Erneuerbaren-Energien-Gesetz" in 2000 [9]. However, the change from conventional power sources to renewable energy sources is not only a simple change of sources in power production. On the contrary, renewable energy sources have entirely different properties regarding power generation than conventional 


\section{Introduction}

sources. They typically produce less and constantly fluctuating power, whereas conventional power sources can provide a constant and controllable power output $[2,4,6,7]$. The change of power generation is thus rather a paradigm change for the entire European power grid, bringing new challenges for power grid operation.

The reason behind the power fluctuations of renewable power sources is that their power output depends critically on external factors such as wind power or solar radiation, which are of course not controllable by power grid operators. Therefore, the power output of renewable energy sources typically strongly fluctuates $[4,10-12$. Furthermore, the possible geographical locations for power plants based on renewable energies depend on geographical factors. This will often lead to a stronger spatial separation between power sources and consumers, for instance in Germany [13]. Power sources based on wind power are typically located in Northern Germany or offshore, where more reliable wind power is present. Solar power plants, on the other hand, are typically located in Southern Germany, in areas with more hours of sunshine. These developments lead to a stronger burden on the existing power grid. For example, transmission lines have to be capable of carrying stronger loads over longer distances in times of high wind and/or solar power.

Furthermore, renewable power sources typically have less power output than conventional power sources [14]. For instance a typical wind farm consists of a few dozen wind turbines, where each turbine has a power output of up to $5 \mathrm{MW}$, whereas a nuclear power plant has an output of around $1 \mathrm{GW}$ [4]. There are only few exceptions such as large offshore wind farms [15] or huge solar parks like Desertec [16]. In order to get the same total power output a large number of renewable power sources is needed to replace one conventional power plant. Because renewable power sources cannot be located everywhere, this process implies decentralized power generation. Decentralization is one of the main developments taking place in today's power grids [17]. This technical term means that electric power is no longer generated in only few power plants which are geographically close to their attached consumers as for example large cities. The opposite of this concept is called centralized power generation. Centralized power grids have the advantage of being in principle easier to control, as power production that takes place close to the consumer does not require a complex topology of the power grid. In contrast, for decentralized power grids, many small power plants are spread out geographically all over the grid. Their locations can be close or far away from large cities. Decentralization will therefore obviously alter the topology of the existing power grid as new transmission lines have to be built and generally a more complex topology of transmission lines is required. In Germany, for instance, new transmission lines are planned from the North to the South to connect wind farms, which are located predominantly in the north, to consumers in the south of Germany according to the grid developing plan ("Netzentwicklungsplan") [18]. The process of decentralization does a priori not necessarily depend on the inclusion of renewable energy sources but, conversely, the inclusion of renewable energies naturally leads to decentralization, because of the aforementioned constraints. This development can be observed in today's power grids. 
As of now, power grids are still dominated by large conventional power plants based on fossil fuel or nuclear material and exhibiting a large power output [4]. Essentially, the effective topology of power grids is locally star-like with transmission lines going from large plants to regional consumers. As more and more renewable power sources contribute, this topology is about to change and power production will become more decentralized and more recurrent [18]. The topologies of current grids vary largely, with large differences, e.g., between grids on islands, such as Great Britain [19], and those in continental Europe, or between areas of different population densities. Decentralization will strongly modify these structures in a yet unknown way. The synchronization dynamics of many power grids with a special topology are well analyzed [20], such as the European power transmission network 21].

In order to get a differentiated view of the so-called Energiewende three major developments must be clearly distinguished: decentralization, spatial separation and fluctuations of the power output. In this thesis we aim at a thorough analysis of the effects of the decentralization process on the stability of power grids. We show that already the decentralization process alone has diverse effects on the stability of future power grids.

Modern power grids are a geographically wide-ranging technical infrastructure consisting of numerous different elements. They can be distinguished into four different interconnected grids with different voltage levels $[22$. The grids with the lowest voltage are called the low-voltage grid. Most consumers obtain electric power from the low-voltage grid, with only few exceptions such as industrial consumers with a high power demand. The middlevoltage grids are fed from transformer stations with the high-voltage grid. Its function is to distribute the power into the low-voltage grids. The high- and maximal-voltage grid connects the power sources with the transformer stations [22. In this thesis we consider the high-voltage grids. The transformer stations between the high and the middle-voltage grid are regarded as consumers.

There exist many fine-tuned operation directives to ensure stable operation of power grids. First of all, power generation and consumption must be balanced at any time, which is achieved by the so-called primary, secondary and tertiary control on the generators' side 23]. Primary control serves to compensate an imbalance between power generation and consumption, where the additional power has to be available on short time scales $(30 \mathrm{~s})$. Secondary control has the same purpose as primary control but with a focus on the singular control areas where the imbalances originated. The additional power has to be available on slightly longer time scales $(5 \mathrm{~min})$. Tertiary control serves mainly for economic optimizations and has to be activated manually 22. Second, the grid must be able to transport the generated power to the consumers. The so-called (N-1)-rule demands that this condition must be satisfied even if a single arbitrary transmission line fails. That is, the load of each transmission line must be smaller than a limiting value in the fully intact network (so-called N-0 case) as well as after a random breakdown of any single element 24]. Furthermore, according to power grid operations, the local voltage and frequency must match the reference values of $230 \mathrm{~V}$ and $50 \mathrm{~Hz}$, respectively, up to 


\section{Introduction}

only small tolerable deviations 25$]$. Constant measurements of the grid frequency serve as the key instrument to maintain stable operation of the grid [25]. If deviations larger than $\pm 0.2 \mathrm{~Hz}$ of the measured frequency to the desired grid frequency of $50 \mathrm{~Hz}$ occur, different countermeasures are executed depending on the size of the deviations 25].

However, although power grids work reliably under normal operating conditions there are still numerous threats such as cataclysms, technical or human failures or even intentional attacks, which can cause large scale power outages with potentially catastrophic consequences and huge economic losses [26]. Still, despite all these threats, the functionality of power grids is often seen as a given fact. But, not surprisingly, sometimes large scale outages happen that affects millions of people. One example is the large scale power outage in the United States and Canada in August 2003, which left 50 million people without electric power for about 16 hours. The largest power outage ever to this date occurred in India in July 2012. It left over 600 million people without electric power for a time span ranging from several hours to more than one day. Another example is the power outage in Western Europe in November 2006, which left approximately 15 million people without electricity for about two hours. The second example is especially interesting, as it was caused by the shutdown of just one transmission line and a following violation of the N-1 safety criterion 27]. This event thus demonstrates that it is in general not always possible to calculate the consequences of failures of certain elements of the power grid and that the technical infrastructure of power grids is still in need of improvements to prevent power outages 28.

To analyze the behavior of power grids under different scenarios different classes of models are in use. We can roughly distinguish between two different classes of models, detailed and abstract. It is virtually impossible to simulate a large-scale power grid including the details of every machine in the grid simply because of the size of the grid. Large modern power grids consist of thousands of different elements and such a model would thus have so many parameters and variables that computer simulations become extremely time consuming, and still insights into the dynamic behavior of power grids would be restricted. Nevertheless, such detailed models which cover every element exist, but they only allow for calculations that represent the behavior of systems of the size of a few streets in a city. However, calculations on this scale are not sufficient to gain insights into the behavior of larger grids. The second class of models are the abstract, large-scale network models. However, although these models are accessible to methods of statistical physics or nonlinear dynamics, they provide only statistical information or a pure directed current (DC) picture. In this thesis we study an oscillator model for power grid operation recently introduced by Filatrella et al. [29]. This model captures the essential dynamical features of a power grid on coarse scales, but is still simple enough to allow for a comprehensive understanding of the fundamental properties of power grid dynamics.

The thesis is organized as follows. The introduction is followed by chapter 4 , which gives an overview over the different existing model classes. We provide an exact derivation of the oscillator model introduced by Filatrella et al. [29] and compare this model to the 
well known Kuramoto model [30]. In chapter 5 we present an analytical investigation of the smallest possible non-trivial power grid within the oscillator model framework, a grid consisting of one generator and one consumer. We demonstrate that already this simple grid contains essential features of power grids, in particular the coexistence of a stable and an unstable state in the parameter space of the model. This feature of coexistence is also present in larger grids and most importantly, also in real-world power grids. In chapter 6 we analyze the effects of decentralization in detail. We demonstrate that for decentralized power grids the onset of stable operation is promoted for smaller transmission capacities. In chapter 7 we provide a detailed analysis of failures of one transmission line. We develop new measures to determine the importance of the failed transmission line for power grid operations. Chapter 8 summarizes the results obtained in this thesis and gives a brief outlook on future research. 



\section{An oscillator model for power grid operation}

In this thesis we consider a power grid model which was recently introduced by Filatrella et al. 29. This model is based on first principle equations of electric circuits of synchronous machines and contains many important parameters and features of power grids and allows for large scale simulations. It is thus valuable for getting insights into a variety of problems regarding the dynamical behavior of power grids under different scenarios.

The first section of this chapter gives an overview over the large variety of different existing power grid models. In particular we discuss different abstract models on the one hand and detailed models on component level mostly used by engineers on the other hand. In the second section we provide a detailed derivation of the Filatrella model. We show the basic first principle equations for electric circuits of synchronous machines and the model's underlying assumptions for power grids of alternating current (AC) of the model. In the third section we discuss the advantages and limitations of this model compared to other models described in the overview section. Finally, we compare the model to the famous Kuramoto model for coupled limit cycle oscillators [30].

\subsection{Overview}

Large modern power grids typically have a geographical span of several thousands kilometers, for instance the European high voltage transmission grid [31]. The total length of transmission lines in the German power grid alone sums up to about 1.7 million kilometers [32]. Modern power grids are therefore often described as the largest man-made machines in existence [20]. Not surprisingly, a rigorous mathematical description of power grids as a whole does not exist. Advanced numerical methods are needed to calculate the voltagecurrent characteristics of larger grids [22]. There exist different model classes with different strengths and weaknesses.

Every generator and consumer is modeled as a node and every transmission line as an edge. Classically, load-flow calculations are used to describe the static operation of power grids. Load-flow calculations determine the voltage and phase of every node in the power grid [22]. With known voltage and phase for every node the active and reactive power of every edge can be determined. There are in principle two different methods in use for 
load-flow calculations 22. The first method is calculating the sums of all currents at every node of the grid. The voltages of the nodes can then be determined via Kirchhoff's circuit laws 33 and the admittances of the grid. For the second method the sum of the power demands of every node are calculated instead of the currents. Kirchhoff's first law can then be formulated for power demands instead of currents. The voltages can then again be calculated by using Kirchhoff's laws and the admittances of the grid. However, these methods can only be used to calculate static states of the grid and do not capture its dynamics. They are thus insufficient for dynamical calculations.

To analyze the structural stability of power grid dynamics often large-scale abstract models are considered. This is especially done to describe cascading failures of transmission lines, which are complicated to describe via load-flow calculations. Examples can be found in 34 37]. These models are applicable for methods of nonlinear dynamics [38]. Although these models provide valuable insights into the behavior of power grids, the principal problem remains that these insights are only of statistical nature or are based on a pure directed current (DC) picture.

Another class are detailed dynamical models on component level. These models are used mostly by engineers for specific simulations. An example can be found in [39]. However, these models require a huge number of parameters and variables. Due to this vast number of parameters and variables it is hard to values gain insights into the dynamic behavior of power grids on large scales which are independent of the specific parameter setting.

\subsection{Oscillator model}

In this section we introduce the power grid model of coupled oscillators by Filatrella et al. [29]. We start with a review of fundamental aspects of AC power systems. We continue with the derivation of the equation of motion of the oscillators from basic equations of synchronous machines. We finally provide an estimate of realistic parameter ranges for the model with regard to real world power grids and compare the model to the famous Kuramoto model.

\subsubsection{Synchronous machines}

A synchronous generator as illustrated schematically in Figure 4.2.1 consists of two essential elements, rotor and stator. The field windings are located on the rotor and the armature winding on the stator. The rotor is driven by a turbine. The three-phase armature windings aa', bb' and cc' are apart by 120 degrees in space to each other. The field winding carries directed current and produces a magnetic field with a north pole $\mathrm{N}$ and a south pole S. The rotating magnetic field induces a three-phase alternating current voltage shifted by 120 degrees in time in the armature windings. The frequency of the induced alternating current 


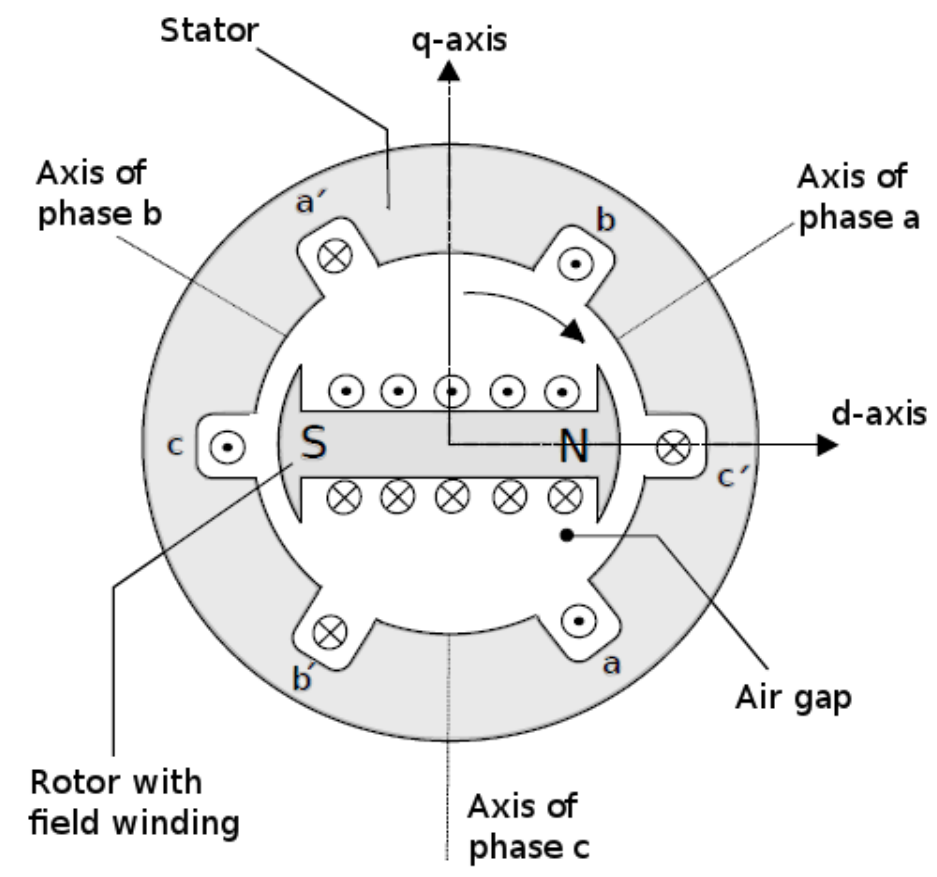

Figure 4.1: Schematic diagram of a three-phase synchronous generator. Figure from 24.

is synchronous to the angular velicity of the rotor, hence the name synchronous machine. The alternating currents in the armature windings induce a rotating magnetic field in the air-gap with the same frequency as the rotor in the steady state. Both magnetic fields interact which each other. The resulting electromagnetic moment of torque counteracts against the rotation of the rotor. To maintain a constant rotation frequency the mechanical moment of torque has to work against this effect. To raise or lower the electrical power output of the synchronous generator the mechanical moment of torque has to be increased or decreased. To operate a synchronous machine as a motor the roles of the electromagnetic and mechanical moment of torque are reversed. Synchronous machines can thus either produce electric power (Generator) or consume electric power (Motor) 24.

\subsubsection{Alternating current}

The operation of almost all modern power grids is based on alternating current. Therefore, we here present the basic definitions and relations in the theory of alternating current. The main feature of alternating current (AC) is time dependent voltage $\tilde{U}(t)$ and current $\tilde{I}(t)$

$$
\begin{aligned}
\tilde{U}(t) & =\hat{U} \cos \left(\omega t+\phi_{u}\right), \\
\tilde{I}(t) & =\hat{I} \cos \left(\omega t+\phi_{i}\right),
\end{aligned}
$$


with amplitudes $\hat{U}$ and $\hat{I}$, frequency $\omega$ and phases $\phi_{u}$ and $\phi_{i}$. It is now useful to switch to complex values for voltage and current. The voltage $\tilde{U}(t)$ and the current $\tilde{I}(t)$ are the real parts of the complex variables

$$
\begin{aligned}
U(t) & =\hat{U} e^{i\left(\omega t+\phi_{u}\right)}, \\
I(t) & =\hat{I} e^{i\left(\omega t+\phi_{i}\right)} .
\end{aligned}
$$

The effective values $\bar{U}$ and $\bar{I}$ are defined as the respective values of directed current, which would produce the same power $P$ at an Ohmic resistance over time [40]. Thus:

$$
P=\overline{U I}=\frac{\bar{U}^{2}}{R}=\frac{1}{T} \int_{0}^{T} \frac{U^{2}(t)}{R} d t=\frac{\hat{U}^{2} e^{i 2 \phi_{u}}}{2 R} .
$$

Hence the effective values are

$$
\bar{U}=\frac{\hat{U}}{\sqrt{2}} e^{i \phi_{u}} \quad \Rightarrow \quad|\bar{U}|=\frac{\hat{U}}{\sqrt{2}}
$$

and

$$
\bar{I}=\frac{\hat{I}}{\sqrt{2}} e^{i \phi_{i}} \quad \Rightarrow \quad|\bar{I}|=\frac{\hat{I}}{\sqrt{2}} .
$$

With $U^{*}(t)$ as the complex conjugate of $U(t)$ we have the following relation:

$$
\begin{aligned}
U(t)+U^{*}(t) & =\hat{U} e^{i\left(\omega t+\phi_{u}\right)}-\hat{U} e^{-i\left(\omega t-\phi_{u}\right)} \\
& =2 \hat{U} \cos \left(\omega t+\phi_{u}\right)=2 \Re(U(t)) .
\end{aligned}
$$

The active power $P$ is defined as the mean over time of the product of the real parts of $U(t)$ and $I(t)[40]$. Hence:

$$
\begin{aligned}
P & =\frac{1}{T} \int_{0}^{T}(\Re(U(t)) \cdot \Re(I(t))) d t \\
& =\frac{1}{T} \int_{0}^{T}\left(\frac{1}{2}\left(U(t)+U^{*}(t)\right) \frac{1}{2}\left(I(t)+I^{*}(t)\right)\right) d t \\
& =\frac{1}{T} \int_{0}^{T}\left(\frac{1}{4}\left(U(t) I^{*}(t)+U^{*}(t) I(t)+U(t) I(t)+U^{*}(t) I^{*}(t)\right)\right) d t \\
& =\frac{1}{T} \int_{0}^{T}\left(\frac{1}{2} \Re\left(U(t) I^{*}(t)+U(t) I(t)\right)\right) d t \\
& =\frac{1}{T} \int_{0}^{T}\left(\frac{1}{2} \Re\left(\hat{U} \hat{I} e^{i\left(\phi_{u}-\phi_{i}\right)}+\hat{U} \hat{I} e^{i\left(2 \omega t+\phi_{u}+\phi_{i}\right)}\right)\right) d t \\
& =\Re\left(\overline{U I^{*}}\right) .
\end{aligned}
$$

The complex power $\bar{S}$ is now defined as $\bar{S}=\overline{U I^{*}}$, such that the active power is the real part of $\bar{S}$ with $P=\Re(\bar{S})$. The imaginary part $\Im(\bar{S})$ is called reactive power $Q$. We thus 
have

$$
\bar{S}=\overline{U I^{*}}=P+i Q
$$

with

$$
P=\Re(\bar{S})=\frac{\hat{U} \hat{I}}{2} \cos \left(\phi_{u}-\phi_{i}\right)
$$

and

$$
Q=\Im(\bar{S})=\frac{\hat{U} \hat{I}}{2} \sin \left(\phi_{u}-\phi_{i}\right)
$$

Active power can be transmitted over long distances and results in grid transmission of energy and can be thus used by consumers. Reactive power is the portion of electricity that establishes and sustains the electric and magnetic fields of alternating-current equipment. Reactive power cannot be transmitted over long distances and cannot be used by consumers [23]. In the following we consider only the active power and discard the reactive power.

\subsubsection{Power transmission in alternating current circuits}

The basic elements of a power grid are generators, consumers and transmission lines. In graph theory every generator and consumer is modeled as a node and every transmission line as an edge. Every node $i$ is then assigned a voltage $U_{i}$ and every edge $(i, j)$ between the nodes $i$ and $j$ an impedance $Z_{i j}$ and a current $I_{i j}$.

In normal operation the magnitude of the voltage amplitude is approximately equal at every node. In fact, strict safety regulations exist for voltage stability 24,41 . To keep the dynamical model as simple as possible we thus assume that

$$
\forall_{i j} \quad \hat{U}_{i}=\hat{U}_{j}=\hat{U}
$$

such that there are only phase differences $\phi_{i j}$ between the nodes. The current $\overline{I_{i j}}$ between two nodes $i$ and $j$ depends on the phase difference of the voltages of these two nodes and the electrical impedance $Z_{i j}$ of the edge:

$$
\begin{aligned}
\overline{I_{i j}} & =\frac{\overline{U_{i}}-\overline{U_{j}}}{Z_{i j}} \\
& =\frac{1}{\sqrt{2}} \frac{\hat{U} e^{i \phi_{i}}-\hat{U} e^{i \phi_{j}}}{Z_{i j}} .
\end{aligned}
$$

The electrical impedance $Z_{i j}$ consists of a real and an imaginary part. The real part is the Ohmic resistance $R_{i j}$ and the imaginary part the reactance $X_{i j}$, consisting of a capacitive and inductive part, such that

$$
Z_{i j}=R_{i j}+i X_{i j}
$$

We further assume that we have no Ohmic losses in transmission lines, such that we have 
for all edges $(i, j)$

$$
R_{i j}=0 .
$$

The current between two nodes (cf. equation 4.12b) now reads

$$
\overline{I_{i j}}=\frac{\hat{U}}{i \sqrt{2} X_{i j}}\left(e^{i \phi_{i}}-e^{i \phi_{j}}\right) .
$$

We model every node as a synchronous machine which produce three-phase alternating current (cf. section 4.2.1). Three-phase alternating current consists of three identical alternating currents of the same frequency, which are phase shifted by 120 degrees. The complex power flow $S_{i j}$ between two nodes is thus (cf. equation 4.8)

$$
\begin{aligned}
S_{i j} & =3 \bar{U}_{i} \cdot \bar{I}_{i j}^{*} \\
& =-\frac{3 \hat{U}}{\sqrt{2}} e^{i \phi_{i}} \frac{\hat{U}}{i \sqrt{2} X_{i j}}\left(e^{-i \phi_{i}}-e^{-i \phi_{j}}\right) \\
& =-\frac{3 \hat{U}^{2}}{i 2 X_{i j}}\left(1-\cos \left(\phi_{i}-\phi_{j}\right)-i \sin \left(\phi_{i}-\phi_{j}\right)\right) .
\end{aligned}
$$

The power flow consists of a real part and an imaginary part, such that

$$
\begin{aligned}
S_{i j} & =P_{i j}+i Q_{i j} \\
& =\frac{3 \hat{U}^{2}}{2 X_{i j}}\left[\sin \left(\phi_{i}-\phi_{j}\right)+i\left(1-\cos \left(\phi_{i}-\phi_{j}\right)\right)\right] .
\end{aligned}
$$

The active power flow is therefore

$$
P_{i j}=\underbrace{\frac{3 \hat{U}^{2}}{2 X_{i j}}}_{P_{i j}^{\text {max }}} \sin \left(\phi_{i}-\phi_{j}\right),
$$

with $P_{i j}^{\max }$ as the new parameter for the maximum transmission capacity of an edge.

\subsubsection{Coupled oscillator model for power grids}

In the model by Filatrella et al. every node of the power grid is modeled as a synchronous machine [29]. Each machine is either a generator or a consumer [24]. Every element $i$ is described by the same equation of motion derived from the dynamics of synchronous machines. Every element has a generalized parameter $P_{i}$, which is the generated or consumed active power. The generated power has a positive value $\left(P_{i}>0\right)$, the consumed power a negative value $\left(P_{i}<0\right)$. The state of each element is determined by its phase angle $\phi_{i}(t)$ and velocity $\dot{\phi}_{i}(t)$. During regular operation, generators as well as consumers within the 


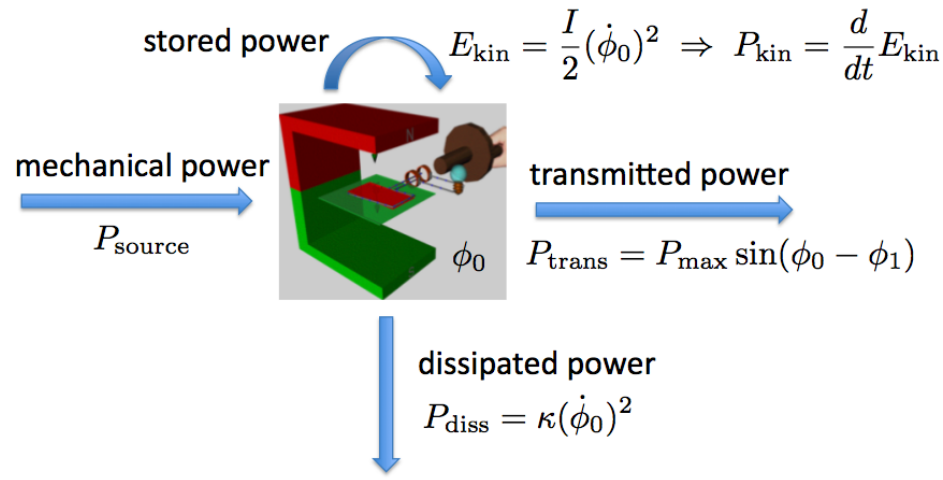

Figure 4.2: Scheme of a synchronous machine. The machine receives input power $P_{\text {source }}$ and stores $\left(P_{\text {kin }}\right)$, dissipates $\left(P_{\text {diss }}\right)$ and transmits $\left(P_{\text {trans }}\right)$ this power. Figure adapted from $[42$.

grid run with the same frequency $\Omega=2 \pi \times 50 \mathrm{~Hz}$ or $\Omega=2 \pi \times 60 \mathrm{~Hz}$. The phase of each element $i$ is then written as

$$
\phi_{i}(t)=\Omega t+\theta_{i}(t),
$$

where $\theta_{i}$ denotes the phase difference to the set value $\omega t$.

The equation of motion for all $\phi_{i}(t)$ can now be obtained from the energy conservation law: The generated or consumed energy $P_{i}^{\text {source }}$ of each single element must equal the energy sum given to or taken from the grid plus the accumulated and dissipated energy of this element (see Figure 4.2). The dissipation power of each element is

$$
P_{i}^{\text {diss }}=\kappa_{i}\left(\dot{\phi}_{i}\right)^{2}
$$

the accumulated power, i.e. the change of the kinetic energy of the rotating synchronous machine,

$$
P_{i}^{\mathrm{acc}}=\frac{1}{2} I_{i} \frac{d}{d t}\left(\dot{\phi}_{i}\right)^{2}
$$

and the transitional active power between two elements is

$$
P_{i j}^{\text {trans }}=P_{i j}^{\max } \sin \left(\phi_{i}-\phi_{j}\right),
$$

see (4.18). The energy conversation law thus reads

$$
P_{i}^{\text {source }}=P_{i}^{\mathrm{diss}}+P_{i}^{\mathrm{acc}}+\sum_{j} P_{i j}^{\mathrm{trans}}
$$

A scheme of the power flow of such a synchronous machine is illustrated in Figure 4.2. The phase difference between two elements is

$$
\phi_{i}(t)-\phi_{j}(t)=\theta_{i}(t)-\theta_{j}(t)
$$

An energy flow between two elements is only possible if there is a phase difference between 
these two. Inserting equations 4.20, 4.21) and 4.22 into equation 4.23 leads to

$$
\begin{aligned}
P_{i}^{\text {source }} & =\kappa_{i}\left(\dot{\phi}_{i}(t)\right)^{2}+\frac{1}{2} I_{i} \frac{d}{d t}\left(\dot{\phi}_{i}(t)\right)^{2}-\sum_{j} P_{i j}^{\max } \sin \left(\theta_{j}(t)-\theta_{i}(t)\right) \\
& =\kappa_{i}\left(\Omega+\dot{\theta}_{i}(t)\right)^{2}+\frac{1}{2} I_{i} \frac{d}{d t}\left(\Omega+\dot{\theta}_{i}(t)\right)^{2}-\sum_{j} P_{i j}^{\max } \sin \left(\theta_{j}(t)-\theta_{i}(t)\right) \\
& =\kappa_{i} \Omega^{2}+2 \kappa_{i} \Omega \dot{\theta}_{i}(t)+\kappa_{i} \dot{\theta}_{i}^{2}(t)+I_{i} \Omega \ddot{\theta}_{i}(t)+I_{i} \ddot{\theta}_{i}(t) \dot{\theta}_{i}(t)-\sum_{j} P_{i j}^{\max } \sin \left(\theta_{j}(t)-\theta_{i}(t)\right) \\
& =\kappa_{i}\left(\Omega^{2}+\dot{\theta}_{i}^{2}(t)\right)+I_{i} \Omega \ddot{\theta}_{i}(t)+\left(2 \kappa_{i} \Omega+I_{i} \ddot{\theta}_{i}(t)\right) \dot{\theta}_{i}(t)-\sum_{j} P_{i j}^{\max } \sin \left(\theta_{j}(t)-\theta_{i}(t)\right) .
\end{aligned}
$$

We can simplify this equation under the assumption of only slow phase changes compared to the frequency $\Omega$, i.e., $\left|\dot{\theta}_{i}\right| \ll \Omega$ and $\left|\ddot{\theta}_{i}\right| \ll \Omega$. The dynamics of the $i$ th machine is then given by

$$
\ddot{\theta}_{i}=\frac{P_{i}^{\text {source }}-\kappa_{i} \Omega^{2}}{I_{i} \Omega^{2}}-\frac{2 \kappa_{i}}{I_{i}} \dot{\theta}_{i}+\sum_{j} \frac{P_{i j}^{\max }}{I_{i} \Omega} \sin \left(\theta_{j}-\theta_{i}\right) .
$$

The elements

$$
K_{i j}=\frac{P_{i j}^{\max }}{I_{i} \Omega}
$$

constitute the connection matrix of the power grid which decodes whether or not there is a transmission line between two elements $(i$ and $j$ ). We assume that we have an equal moment of inertia $I_{i}$ for all nodes $i$, such that $K_{i j}=K_{j i}$ holds. For convenience we define the new parameters $P_{i}$ and $\alpha_{i}$

$$
P_{i}=\frac{P_{i}^{\text {source }}-\kappa_{i} \Omega^{2}}{I_{i} \Omega}
$$

and

$$
\alpha_{i}=\frac{2 \kappa_{i}}{I_{i}}
$$

The equation of motion than assumes the simple form

$$
\frac{d^{2} \theta_{i}}{d t^{2}}=P_{i}-\alpha_{i} \frac{d \theta_{i}}{d t}+\sum_{j} K_{i j} \sin \left(\theta_{j}-\theta_{i}\right) .
$$

Under the assumption of $\alpha_{i}=\alpha_{j}=\alpha$ for all $i$ and $j$ we rescale the equation with $s=\alpha t$ and new variables $\tilde{P}=P / \alpha^{2}$ and $\tilde{K}=K / \alpha^{2}$. This leads to

$$
\frac{d^{2} \theta_{i}}{d s^{2}}=\tilde{P}_{i}-\frac{d \theta_{i}}{d s}+\sum_{j} \tilde{K}_{i j} \sin \left(\theta_{j}-\theta_{i}\right)
$$


In the steady state both derivatives $\frac{d \theta_{i}}{d s}$ and $\frac{d^{2} \theta_{i}}{d s^{2}}$ are zero, such that

$$
0=P_{i}+\sum_{j} K_{i j} \sin \left(\theta_{j}-\theta_{i}\right)
$$

holds for each element. For the sum over all equations, one for each element $i$, the following holds

$$
-\sum_{i} P_{i}=\sum_{i<j} K_{i j} \sin \left(\theta_{j}-\theta_{i}\right)+\sum_{i>j} K_{i j} \sin \left(\theta_{j}-\theta_{i}\right)=0,
$$

because $K_{i j}=K_{j i}$ and the sin-function is antisymmetric. This means it is a necessary condition that the sum of the generated power $\left(P_{i}>0\right)$ equals the sum of the consumed power $\left(P_{i}<0\right)$ in the steady state.

\subsubsection{Parameter setting}

The equation of motion 4.30 contains three types of parameters: the produced or consumed power $P_{i}$ and the damping $\alpha$ and the transmission capacity $K_{i j}$ of edge $i j$. In the following numerical examples we assume that large centralized power plants generate $P_{i}^{\text {source }}=100 \mathrm{MW}$ each 43, 44. A synchronous generator of this size would have a moment of inertia of the order of $I_{i}=10^{4} \mathrm{~kg} \mathrm{~m}^{2}, 20,43,44$. The mechanically dissipated power $\kappa_{i} \Omega^{2}$ usually is a small fraction of $P^{\text {source }}$ only. A major overhead power line can have a transmission capacity of up to $P_{i j}^{\max }=700 \mathrm{MW}$. We take $\Omega=2 \pi \times 50 \mathrm{~Hz}$. This leads to the following estimates for $P_{i}, \alpha$ and $K_{i j}$ :

$$
\begin{aligned}
P_{i} & =\frac{95 \mathrm{MW}}{2 \pi \times 50 \mathrm{~Hz} \times 10^{4} \mathrm{~kg} \mathrm{~m}^{2}} \\
& \approx 30 \frac{1}{\mathrm{~s}^{2}}, \\
\alpha & =\frac{2 \times 5 \mathrm{MW}}{10^{4} \mathrm{~kg} \mathrm{\textrm {m } ^ { 2 }} \times(2 \pi \times 50 \mathrm{~Hz})^{2}} \\
& \approx 0.02 \frac{1}{\mathrm{~s}} .
\end{aligned}
$$

and

$$
\begin{aligned}
K_{i j} & =\frac{700 \mathrm{MW}}{10^{4} \mathrm{~kg} \mathrm{~m}{ }^{2} \times 2 \pi \times 50 \mathrm{~Hz}} \\
& \approx 200 \frac{1}{\mathrm{~s}^{2}}
\end{aligned}
$$


However, in a realistic power grid there are additional sources of dissipation, especially Ohmic losses and losses caused by damper windings [25], which are not taken into account directly in the coupled oscillator model. Therefore, for our simulations we take a higher value for $\alpha$ of $\alpha=0.1 \mathrm{~s}^{-1}$ and a smaller value for $P$ of $P_{i}=10 \mathrm{~s}^{-2}$ for large power plants. Decentralized sources are characterized by lower values of $P_{i}$. For a typical consumer we assume $P_{i}=-1 s^{-2}$, corresponding to a small city. A transmission line connecting a small city to the grid usually has a smaller transmission capacity, such that $K_{i j}<10^{2} \mathrm{~s}^{-2}$ is realistic. These values are in the order of magnitude commonly used in the literature 19 , 29.

\subsubsection{Comparison with Kuramoto model}

The Kuramoto model for coupled oscillators was introduced by Y. Kuramoto in 1984 [30]. It is based on an idea by A. Winfree [45]. It considers $N$ coupled oscillators with their dynamics governed by the set of equations

$$
\dot{\theta}_{i}=\omega_{i}+\sum_{j=1}^{N} \Gamma_{i j}\left(\theta_{j}-\theta_{i}\right), \quad i=1, \ldots ., N .
$$

Here $\theta_{i}$ oscillator $i$ 's phase, $\omega_{i}$ its intrinsic frequency and $\Gamma_{i j}$ is the coupling strength between oscillators $i$ and $j$. This model has proven to be useful for analyzing a huge number of phenomena [46]. They reach from synchronizing behavior of light flashing fireflies [47] to the synchronizing footsteps of humans walking across the Millennium Bridge in London [48. There is rich literature in the field, for an overview see e.g. [49]. However, the obvious difference between the model derived by Filatrella et al. and the Kuramoto model is that the former contains second order oscillators while equation (4.37) is of first order. There is much less knowledge about the synchronization behavior of damped second order oscillators compared to the insights gained on the Kuramoto model [46].

The model introduced by Filatrella et al. essentially combines the swing equation of a synchronous machine [50] with damping $D_{i}$, angular momentum $M_{i}$ and the difference of mechanical and electrical power, $P_{m}-P_{e}$,

$$
M_{i}=\frac{d^{2} \theta_{i}}{d t^{2}}+D_{i} \frac{d \theta_{i}}{d t}=P_{m}-P_{e}
$$

with active power transmission derived from the dynamics of alternating currents (AC) as the coupling term (cf. section 4.2.3). It was only recently shown by Dörfler et al. [51] that interconnected swing equations [50] for oscillators can be expressed via the non-uniform Kuramoto model. This comparison has drawn a lot of interest in the last years in the scientific community especially with regards to synchronization. Buzna et al. [52] analyzed the synchronization dynamics of populations of Kuramoto oscillators with two different frequencies, especially the minimal required coupling strength between the oscillators for 
global synchronization. Other examples for the relation between interconnected swing equations and Kuramoto oscillators can be found in [20, 53 56]. In summary, different models for the study of interconnected swing equations are available. In this thesis we choose to directly study the model introduced by Filatrella et al. [29].

\subsection{Discussion}

In this chapter we introduced the model derived by Filatrella et al. 29] on which the further analysis in this thesis is based. The model is based on the dynamics of synchronous machines. The coupling term for active power transmission between two nodes of the grid is derived from circuits of alternating current. To derive the equation of motion of the oscillator model we made two major assumptions. The first is that all elements of the power grid can be described as a network of synchronous machines. The second is that we have no Ohmic losses in transmission lines. Both assumptions are not completely fulfilled in real world power grids. For instance, solar power plants cannot be described as synchronous machines and Ohmic losses are present in real transmission lines. But, with the main exception of solar power plants, most other power plants can be described as synchronous machines, which are the vast majority. Ohmic losses are indeed small due to high-voltage transmission [40], such that this assumption is common in the literature, see for example [57. Therefore, although the model has its limitations, it still provides a reasonable description of real world power grids.

Furthermore, we used a homogeneous parameter setting. We assumed that all transmission lines have the same capacity, with the exception of those directly connected to a power source. These are modeled with a higher capacity to avoid trivial overloads. Every consumer uses an equal amount of power; similarly, each small (large) generator produces the same amount of power as every other small (large) generator. The goal of this thesis is to gain insights into the principal behavior of large power grids depending on the network topology, particularly their capability to synchronize. This can be seen most clearly for a homogeneous set of parameters. An application to the heterogeneities of real-world grids deserves further studies, but we are confident that our results can serve as a guideline for such a study.

However, it is very hard to derive a realistic power grid model which captures all features of real world power grids and is still applicable for large scale simulations. Models which capture all these features are limited to the scale of very few generators and transmission lines. The model by Filatrella et al. is applicable for large scale simulations and captures the dynamics of the power grid. The model is therefore useful to get ideas how real power grids behave under certain scenarios. It is of course necessary to test these ideas with real data in concrete situations. 



\section{Dynamics \& self-organization of an elementary model}

In this chapter we present and analyze an elementary example model of a power grid consisting of only two elements, a generator and a consumer. The steady state representing normal operation of this system is analytically derived. Although it is the simplest possible system, already this simple system reveals the existence of three different stability regimes that are also present in larger model systems later analyzed in this thesis.

First we set up the effective equations of motion for this system. Using these equations we demonstrate that deviations to the grid frequency $\omega$ already occur if the condition of equal power production and consumption is violated. We thus show that the measurement of these deviations is a way to check the power balance and a potential guideline to power adaptation processes. Two steady states exist if the transmitted active power does not exceed the transmission capacity of the line connecting generator and consumer: a linear stability analysis (e.g. [59]) shows that the system has a stable as well as an unstable fixed point. The stable fixed point allows stable operation of the power grid. Furthermore, we show that the system has three different stability regimes in its parameter space, ranging from a globally stable to a globally unstable region with an additional region of coexistence of both states. We argue that real power grids operate in the region of coexistence such that their dynamics and stability sensitively depend on the initial state. Thus, a purely static evaluation of the operation of a power grid, as being common in many flow calculations of engineering is incomplete.

Notably, the effective equations of motion of the example system can be mapped exactly to the motion of a particle in a tilted washboard potential. This mapping provides a clear illustration of the three different stability regimes in the parameter space of the system. Finally, we discuss how the balance of generated and consumed power, a necessary condition for a stable operation at the grid reference frequency, can be achieved by different control schemes based on local frequency measurements. The chapter closes with a brief summary and outlook.

Parts of this chapter have been previously published in 19,58 . 


\subsection{Effective equations of motion}

In the following we analyze a power grid consisting of a generator, a consumer and a transmission line connecting both with capacity $K$. The generator produces the power $P_{1}>0$. The consumer consumes the power $P_{2}<0$. This power grid can only operate in a stable manner if the power is balanced, i.e., $P_{1}=-P_{2}$ as shown in section 4.2.4 ( see (4.33)). The equations of motion 4.30 for both the generator and the consumer are now:

$$
\begin{aligned}
& \ddot{\theta}_{1}=P_{1}-\alpha \dot{\theta}_{1}+K \sin \left(\theta_{2}-\theta_{1}\right) \\
& \ddot{\theta}_{2}=P_{2}-\alpha \dot{\theta}_{2}+K \sin \left(\theta_{1}-\theta_{2}\right) .
\end{aligned}
$$

I is convenient to reduce these equations of second order oscillators to equations of first order by introducing the new variables $\chi_{i}:=\dot{\theta}_{i}$, i.e.,

$$
\begin{aligned}
\dot{\chi}_{1} & =P_{1}-\alpha \chi_{1}+K \sin \left(\theta_{2}-\theta_{1}\right) \\
\dot{\chi}_{2} & =P_{2}-\alpha \chi_{2}+K \sin \left(\theta_{1}-\theta_{2}\right) \\
\dot{\theta}_{1} & =\chi_{1} \\
\dot{\theta}_{2} & =\chi_{2} .
\end{aligned}
$$

We simplify this set of equations by taking both the difference and the sum between the first two and the last two equations respectively. With $\Delta P=P_{2}-P_{1}=2 P_{2}$, the phase difference $\Delta \theta=\theta_{2}-\theta_{1}$, the velocity difference $\Delta \chi=\chi_{2}-\chi_{1}$, the sum of the velocities $\sum \chi=\chi_{1}+\chi_{2}$ and the sum of the phases $\sum \theta=\theta_{1}+\theta_{2}$ the equations read

$$
\begin{aligned}
\Delta \dot{\chi} & =\Delta P-\alpha \Delta \chi-2 K \sin \Delta \theta \\
\Delta \dot{\theta} & =\Delta \chi \\
\sum \dot{\chi} & =-\alpha \sum \chi \\
\sum \dot{\theta} & =\sum \chi .
\end{aligned}
$$

The first two equations are the effective equations of motion of the system as $\sum \chi$ evolves to the steady state $\sum \chi=0$ and thus also $\sum \theta$ reaches a steady state. 


\subsection{Bifurcation structure}

In a steady state both derivatives $\Delta \dot{\chi}$ and $\Delta \dot{\theta}$ must be zero. The effective equations of motion thus read:

$$
\begin{aligned}
& 0=\Delta P-\alpha \Delta \chi-2 K \sin \Delta \theta \\
& 0=\Delta \chi
\end{aligned}
$$

These equations have two solutions for $\Delta P<2 K$, such that the system has two fixed points $T_{1}$ and $T_{2}$ in the steady state for $\Delta P<2 K$, which are analyzed in detail below. The two fixed points of the effective equations of motion (5.3) are in terms of the phase difference $\Delta \theta$ and the velocity difference $\Delta \chi$ :

$$
T_{1}:=\left(\begin{array}{c}
\Delta \chi_{1}^{*} \\
\Delta \theta_{1}^{*}
\end{array}\right)=\left(\begin{array}{c}
0 \\
\arcsin \frac{\Delta P}{2 K}
\end{array}\right)
$$

and

$$
T_{2}:=\left(\begin{array}{c}
\Delta \chi_{2}^{*} \\
\Delta \theta_{2}^{*}
\end{array}\right)=\left(\begin{array}{c}
0 \\
\pi-\arcsin \frac{\Delta P}{2 K}
\end{array}\right) .
$$

For $\Delta P>2 K$ no fixed point exists, because the arcsin function has no solution for $\Delta P>$ $2 K$. The critical coupling strength $K_{c}$ for the existence of a fixed point is therefore

$$
K_{c}=\frac{\Delta P}{2} .
$$

At the critical point, for $\Delta P=2 K$ only one fixed point exists, $T_{1}=T_{2}$, at

$$
\left(\begin{array}{c}
\Delta \chi^{*} \\
\Delta \theta^{*}
\end{array}\right)=\left(\begin{array}{c}
0 \\
\frac{\pi}{2}
\end{array}\right)
$$

Stable operation of this simple power grid is enabled by the existence of a stable fixed point. The local stability of the two fixed points existing for $K>K_{c}=\Delta P / 2$ is determined by the eigenvalues of the Jacobian of the dynamical system (5.3), given by

$$
J=\left(\begin{array}{cc}
-\alpha & -2 K \cos \Delta \theta^{*} \\
1 & 0
\end{array}\right)
$$

The eigenvalues $\lambda_{ \pm}^{*}$ of $J$ are:

$$
\lambda_{ \pm}^{*}=-\frac{\alpha}{2} \pm \sqrt{\frac{\alpha^{2}}{4}-2 K \cos \Delta \theta^{*}}
$$




\section{Dynamics \& self-organization of an elementary model}

Applying the trigonometric identity $\cos ^{2}=1-\sin ^{2}$ and using (5.5) and (5.6) this yields

$$
\begin{aligned}
\lambda_{ \pm}^{(1)} & =-\frac{\alpha}{2} \pm \sqrt{\frac{\alpha^{2}}{4}-\sqrt{4 K^{2}-\Delta P^{2}}} \\
& =: \frac{\alpha}{2} \pm \sqrt{D}
\end{aligned}
$$

with $D=\frac{\alpha^{2}}{4}-\sqrt{4 K^{2}-\Delta P^{2}}$ at the first fixed point $T_{1}$ and

$$
\lambda_{ \pm}^{(2)}=-\frac{\alpha}{2} \pm \sqrt{\frac{\alpha^{2}}{4}+\sqrt{4 K^{2}-\Delta P^{2}}}
$$

at the second fixed point $T_{2}$, respectively. The eigenvalues at the critical point $K_{c}$ with $\Delta P=2 K$ are thus $\lambda_{+}=0$ and $\lambda_{-}=-\alpha$. A nonlinear stability analysis would thus be necessary in order to determine the stability of the fixed point in this marginal case.

Depending on $K$, the eigenvalues at $T_{1}$ are either both real and negative or complex with negative real values. One eigenvalue at $T_{2}$ is always real and positive, the other one real and negative. Thus only $T_{1}$ is stable and enables a stable operation of the power grid. It has real and negative eigenvalues if $K \in\left(K_{c}, K_{2}\right)$ where $K_{2}$ is defined by $D>0$ in $5.11 \mathrm{~b}$, such that

$$
K_{2}=\sqrt{\frac{\alpha^{4}}{64}+\frac{\Delta P^{2}}{4}}=\frac{\Delta P}{2} \sqrt{\frac{\alpha^{4}}{16 \Delta P^{2}}+1} .
$$

Thus $K \in\left(K_{c}, K_{2}\right)$ is only possible for $\alpha$ considerably larger than 1 . For $K \geq K_{2}$ the eigenvalues of $T_{1}$ are complex with a negative real value

$$
\Re\left(\lambda_{ \pm}^{(1)}\right)=-\frac{\alpha}{2}
$$

for which the power grid exhibits damped oscillations around the fixed point. As power grids should work with only minimal dissipation $(\alpha \leq 1)$ and thus $K \geq K_{2}$, this is the practically relevant setting.

Stable operation of the power grid is guaranteed by the existence of a globally stable fixed point. The fixed point is globally stable if the change in the energy $E$ of the system averaged over one period $T$ is negative, i.e., $\left\langle\frac{d E}{d t}\right\rangle<0$, such that the trajectories always converge to the stable fixed point [60]. Otherwise a limit cycle coexists with the fixed point [60]. An analytical approximation for the border between the globally stable and the coexistence regime can be obtained in the low-friction limit [60]. Taking the difference of both equations (5.1) with $x(t):=\Delta \theta(t)$ yields

$$
\begin{aligned}
\ddot{x} & =\Delta P-2 K \sin (x)-\alpha \dot{x} \\
& =-\frac{\partial E}{\partial x}+\Delta P-\alpha \dot{x}
\end{aligned}
$$


with $\Delta P$ as the acceleration and $\dot{x}$ as the friction of the system. The energy $E$ of the system is defined as

$$
E=\frac{(\dot{x})^{2}}{2}-2 K \cos (x)
$$

with the potential $-2 K \cos (x)$. The change of energy is thus

$$
\frac{d E}{d t}=\ddot{x} \dot{x}+2 K \sin (x) \dot{x} .
$$

Inserting equation (5.15a) into (5.17) yields

$$
\begin{aligned}
\frac{d E}{d t} & =(\Delta P-2 K \sin (x)-\alpha \dot{x}) \dot{x}+2 K \sin (x) \dot{x} \\
& =\Delta P \dot{x}-\alpha(\dot{x})^{2} .
\end{aligned}
$$

The condition for the border between the globally stable and the coexistence regime is given by

$$
\frac{\overline{d E}^{T}}{d t}=0
$$

Hence

$$
\Delta P \overline{\dot{x}}^{T}-\alpha{\overline{\dot{x}^{2}}}^{T}=0
$$

is the condition for the border between the globally stable and the coexistence regime. The period $T$ is defined by 60 :

$$
T=\int_{0}^{T} d t=\int_{-\pi}^{\pi} \frac{1}{\dot{x}} d x
$$

We can now calculate

$$
\overline{\dot{x}}^{T}=\frac{1}{T} \int_{0}^{T} \dot{x} d t=\frac{1}{T} \int_{-\pi}^{\pi} d x=\frac{2 \pi}{T}
$$

and

$$
\begin{aligned}
{\overline{\dot{x}^{2}}}^{T} & =\frac{1}{T} \int_{0}^{T} \dot{x}^{2} d t=\frac{1}{T} \int_{-\pi}^{\pi} \dot{x} d x \\
& =\frac{1}{T} \int_{-\pi}^{\pi} \sqrt{2 E+4 K \cos (x)} d x .
\end{aligned}
$$

For the critical line we hence find (see 5.20 )

$$
\Delta P \frac{2 \pi}{T}=\frac{\alpha}{T} \int_{-\pi}^{\pi} \sqrt{2 E+4 K \cos (x)} d x .
$$

The energy of the power grid on the border between the globally stable and the coexistence regime in the low-friction limit is the maximum of the potential, i.e., $2 K$. If the power grid has a smaller energy in the low-friction limit the fixed point is globally stable, otherwise the power grid is in the coexistence regime. To approximate the border between these two 


\section{Dynamics \& self-organization of an elementary model}

regimes we can thus simplify the integral in equation 5.24 in the low friction limit with $E=2 K$ :

$$
\int_{-\pi}^{\pi} \sqrt{4 K+4 K \cos (x)} d x=2 \sqrt{K} \int_{-\pi}^{\pi} \sqrt{1+\cos (x)} d x .
$$

With $u:=\frac{x}{2}$ and the trigonometric identity $\cos (2 u)=\cos ^{2}(u)-\sin ^{2}(u)$ we can solve the integral (5.25):

$$
\begin{aligned}
2 \sqrt{K} \int_{-\pi}^{\pi} \sqrt{1+\cos (x)} d x & =2 \sqrt{K} \int_{-\frac{\pi}{2}}^{\frac{\pi}{2}} \sqrt{1+\cos (2 u)} 2 d u \\
& =4 \sqrt{K} \int_{-\frac{\pi}{2}}^{\frac{\pi}{2}} \sqrt{1+\cos ^{2}(u)-\sin ^{2}(u)} d u \\
& =4 \sqrt{K} \int_{-\frac{\pi}{2}}^{\frac{\pi}{2}} \sqrt{2 \cos ^{2}(u)} d u \\
& =4 \sqrt{K} \cdot \sqrt{2} \int_{-\frac{\pi}{2}}^{\frac{\pi}{2}} \cos (u) d u \\
& =4 \sqrt{2 K}[\sin (u)]_{-\frac{\pi}{2}}^{\frac{\pi}{2}}=8 \sqrt{2 K} .
\end{aligned}
$$

We thus find for the low-friction approximation the following border between the globally stable and the coexistence regime (cf. (5.24))

$$
\frac{\Delta P}{2 K}=\frac{2 \cdot \sqrt{2}}{\pi} \cdot \frac{\alpha}{\sqrt{K}}
$$

The excellent agreement of the low-friction approximation (blue line) for $\alpha / \sqrt{K}<0.6$ with the numerically calculated border (black curve) separating the two regimes is illustrated in Figure 5.1 .

\subsection{Global stability properties}

In the last section we have shown that the simple model of a power grid has different stability properties for different parameter settings. The different stability regimes are illustrated in Figure 5.1 for the parameter space of the system. In the upper region of Figure 5.1 for $\frac{\Delta P}{2 K}>1$ no steady state exists (cf. section 5.2). The physical reason is that the load exceeds the capacity of the transmission line. No stable operation is possible and all trajectories converge to a limit cycle. In real-world power grids the limit cycle cannot be reached, because an overload of transmission lines leads to a power outage.

For $\frac{\Delta P}{2 K}<1$ two different regimes exist, a regime where only the fixed points exist and a coexistence regime where both the fixed points and the limit cycle exist (cf. section 5.2). The regime with the fixed point only covers the area on the right hand side of the border (black curve) in the lower region of parameter space (cf. Figure 5.1). In this regime all 


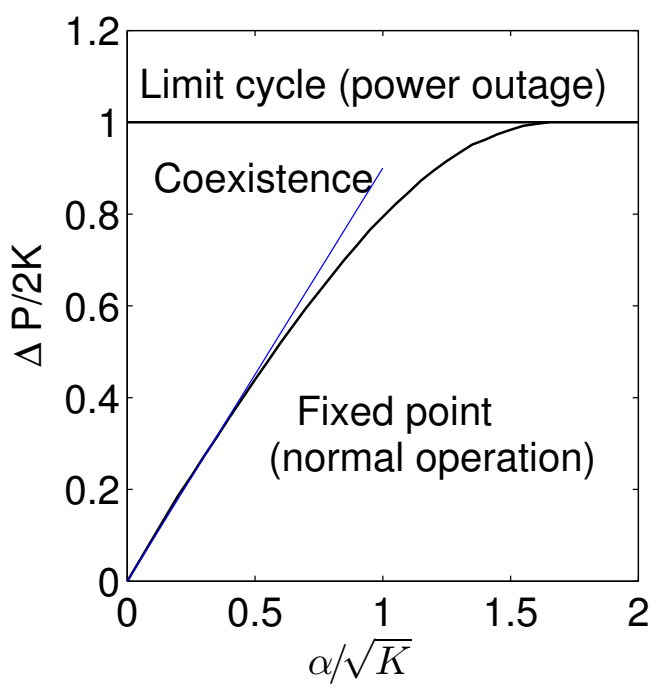

Figure 5.1: Stability phase diagram in parameter space. Blue line: low-friction approximation for the border between the globally stable and the coexistence regime.

trajectories converge to the fixed point and the system is globally stable. The reason is that the high damping $\alpha$ in this regime slows down the dynamics independently of the initial conditions.

Most interestingly, in the remaining region of parameter space, the fixed point and the limit cycle coexist. In the coexistence regime the dynamics depends crucially on the initial conditions. The trajectories can converge either to the fixed point or the limit cycle. Practically, this is the most relevant case. Power systems are optimized to have low dissipation [25] and thus a low damping rate $\alpha$ in the considered model. In fact, damped oscillations towards the stable fixed point are regularly observed in real power grids [25].

Exemplary trajectories with two different initial conditions are illustrated along with the phase portrait of all three regions of the parameter space in Figure 5.2. The dynamics of the globally unstable regime with the limit cycle depicted as a red line are illustrated in Figure $5.2(\mathrm{a})$. The evolution of the phase difference $\Delta \theta$ and phase derivative difference $\Delta \chi$ is illustrated in Figure 5.2 (b,c) for two exemplary trajectories with different initial conditions, $(\Delta \chi, \Delta \theta / 2 \pi)=(0,0.5)$ (dashed red line) and $(\Delta \chi, \Delta \theta / 2 \pi)=(0,0.1)$ (solid blue line). Both trajectories converge to the limit cycle. The phase difference $\Delta \theta$ is constantly growing and the phase derivative difference $\Delta \chi$ is constantly oscillating for both trajectories. The system is thus incapable of reaching a steady state in this region of parameter space.

The phase portrait of the globally stable regime is illustrated in Figure $5.2(\mathrm{~d})$. The stable fixed point is marked with a red cross. The evolution of the phase difference $\Delta \theta$ and phase derivative difference $\Delta \chi$ for two exemplary trajectories starting from same initial conditions as above is illustrated in Figure $5.2(\mathrm{e}, \mathrm{f})$. Both trajectories converge into the stable fixed 


\section{Dynamics \& self-organization of an elementary model}
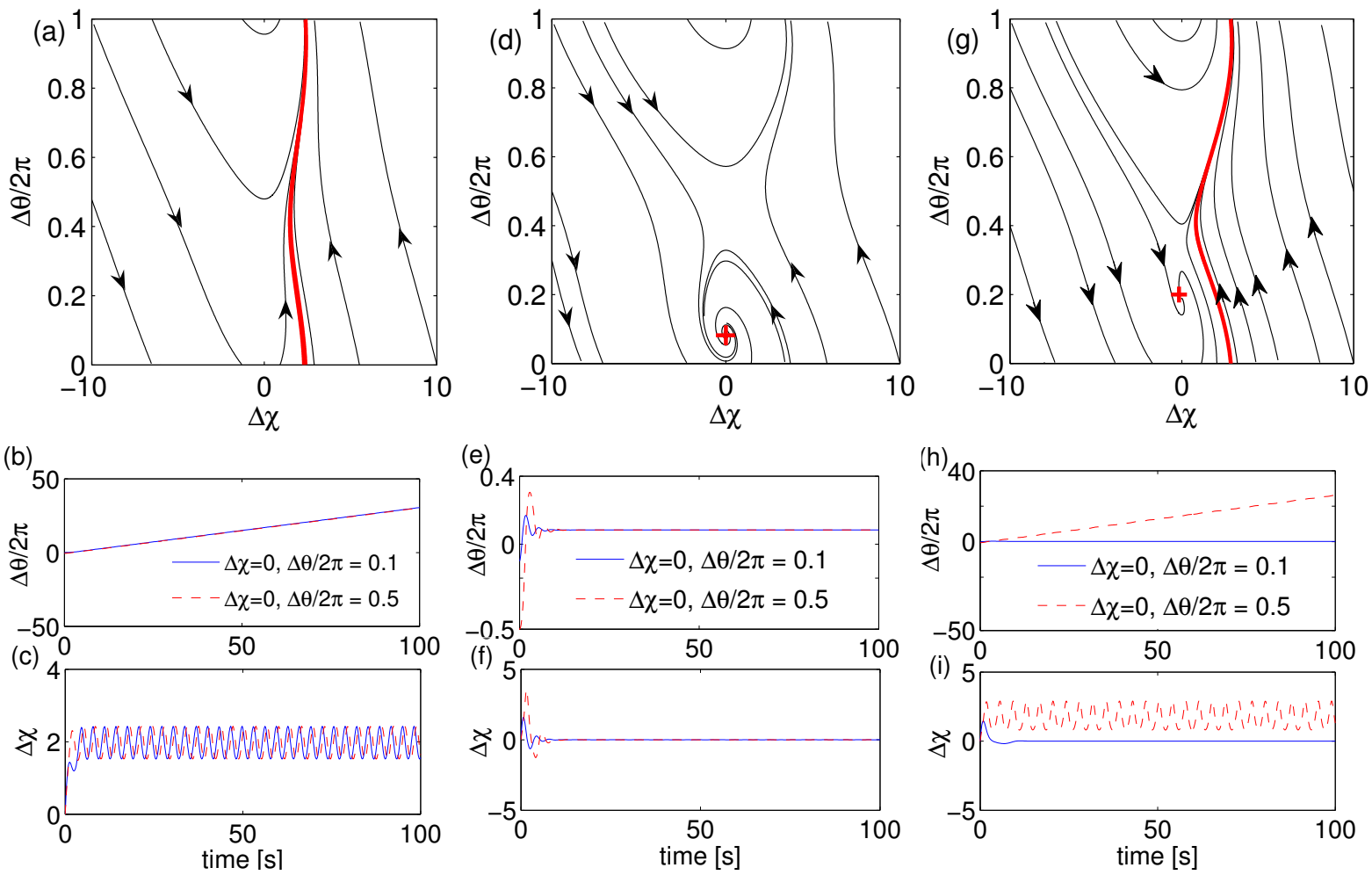

Figure 5.2: Dynamics of an elementary network with one generator and one consumer $(\alpha=$ $\left.1 \mathrm{~s}^{-1}\right)$. Initial Conditions: blue: $(\Delta \chi, \Delta \theta / 2 \pi)=(0,0.1)$, red: $(\Delta \chi, \Delta \theta / 2 \pi)=(0,0.5)$. (a,b,c) Globally stable limit cycle for $\Delta P=2 \mathrm{~s}^{-2}$ and $K=0.5 \mathrm{~s}^{-2}$. Both example trajectories converge to the limit cycle. (d,e,f) Globally stable phase locking (normal operation) for $\Delta P=2 \mathrm{~s}^{-2}$ and $K=2 \mathrm{~s}^{-2}$. Both example trajectories converge into the stable fixed point. (g,h,i) Coexistence of phase locking and limit cycle for $\Delta P=2 \mathrm{~s}^{-2}$ and $K=1.1 \mathrm{~s}^{-2}$. The trajectory with initial condition $(\Delta \chi, \Delta \theta / 2 \pi)=(0,0.1)$ converges into the fixed point while the trajectory with initial condition $(\Delta \chi, \Delta \theta / 2 \pi)=(0,0.5)$ into the limit cycle.

point. The phase derivative difference thus goes to zero and the phase difference has a fixed value. The system always converges to its stable state for all initial conditions.

The phase portrait of the coexistence regime is illustrated in Figure 5.2(g). The phase portrait contains both the limit cycle and the stable fixed point, which are depicted by the red line and the red cross, respectively. The evolution of the phase differences and phase derivative differences for two trajectories again with the same initial conditions is illustrated in Figure $5.2(\mathrm{~h}, \mathrm{i})$. The trajectory with initial conditions $(\Delta \chi, \Delta \theta / 2 \pi)=(0,0.1)$ (solid blue line) converges to the stable fixed point, the trajectory with initial conditions $(\Delta \chi, \Delta \theta / 2 \pi)=(0,0.5)$ (dashed red line) converges to the limit cycle. Therefore the phase derivative difference is either going to zero for the trajectory that converges to the stable fixed point or is fluctuating for the trajectory converging to the limit cycle. Consequently, the phase difference has a constant value or is constantly growing. Which behavior the 
system exhibits depends crucially on the initial conditions.

We can thus conclude that if the power grid is in the coexistence regime of parameter space and its stable state is perturbed, it is not a priori certain that the system can reach the stable fixed point again after the perturbation is removed. On the contrary, the system's further behavior depends on the position of its state in phase space. If the current lies still in the basin of attraction of the stable fixed point after the perturbation is removed it converges back into the stable fixed point of normal operation, otherwise it converges into the limit cycle and we thus have a power outage. A perturbation can for instance occur because the condition $P_{1}=-P_{2}$ is violated for a certain period of time. Another possible perturbation could be the change of the value of the transmission capacity $K$ in one or more transmission lines. In case of such an event, the structure of parameter space changes - in particular the position of the stable fixed point in parameter space and the corresponding basin of attraction. Therefore, the capability of the system to maintain its stable state depends on its current state.

Most real power grids are operating in the region of coexistence. Perturbations frequently occur in real-world power grids, but most of the time power grids are capable of preserving stable operation. Nevertheless, power outages occur from time to time. There are many examples for outages in real world power grids, one of them is the well known power outage in November 2006 in Western Europe, caused by the cutting of one transmission line in Germany [27]. The power grid model that we have introduced in this section contains this important feature of real-world power grids, the coexistence of a stable and an unstable state.

\subsection{Equivalence to the dynamics in a tilted washboard potential}

The system of one generator and one consumer can be mapped to the Newtonian motion of a particle in a tilted washboard potential [43]. In order to demonstrate this equivalence we directly subtract the two equations of (5.1) from each other,

$$
\ddot{\theta}_{2}-\ddot{\theta}_{1}=P_{2}-P_{1}-\alpha\left(\dot{\theta}_{2}-\dot{\theta}_{1}\right)-2 K \sin \left(\theta_{2}-\theta_{1}\right) .
$$

With $\Delta \theta:=\theta_{2}-\theta_{1}$ this leads to

$$
\Delta \ddot{\theta}=\Delta P-\alpha \Delta \dot{\theta}-2 K \sin (\Delta \theta) .
$$

Through a rescaling of time by $\tau=\alpha t$ together with the introduction of new variables, $\Delta p:=\frac{\Delta P}{\alpha^{2}}, k:=\frac{K}{\alpha^{2}}$ and $x(\tau):=\Delta \theta(\tau)$ the equation of motion becomes

$$
\ddot{x}+\dot{x}+2 k \sin (x)-\Delta p=0 .
$$




\section{Dynamics \& self-organization of an elementary model}
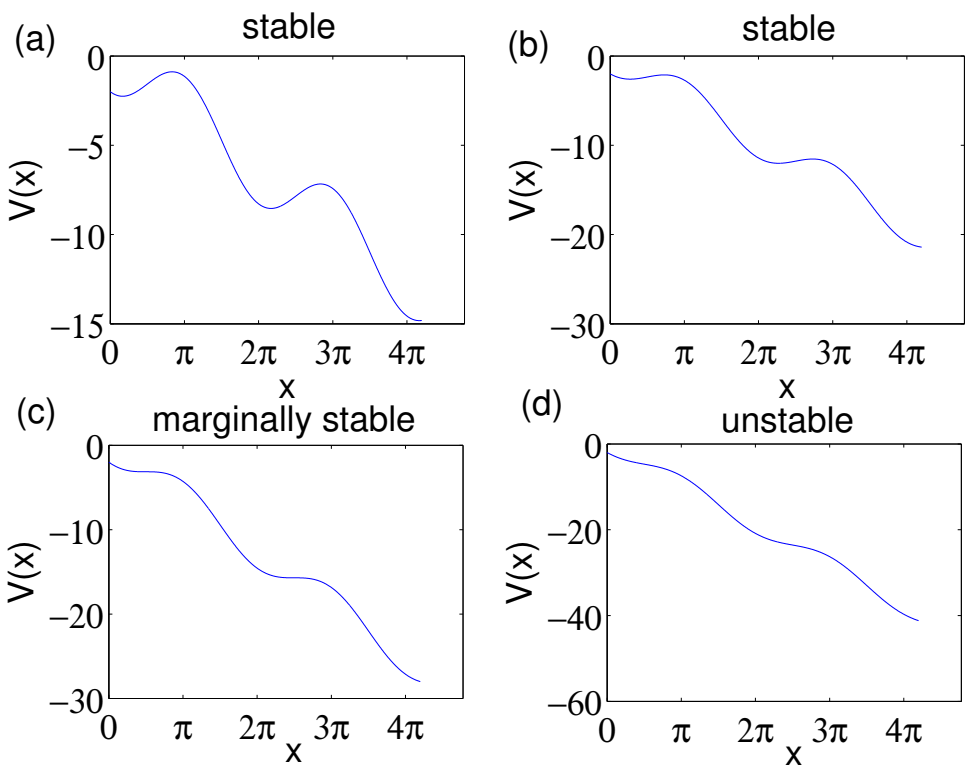

Figure 5.3: The potential $V(x)=-\Delta p x-2 k \cos (x)$ for $k=1$ and different values of $\Delta p$. (a) $\Delta p=1$, (b) $\Delta p=1.5$, (c) $\Delta p=2$ and (d) $\Delta p=3$.

This equation can now also be regarded as the equation of motion for a particle in a tilted washboard potential,

$$
\ddot{x}=-\dot{x}-\frac{d V}{d x},
$$

with the potential $V(x)$

$$
V(x)=-\Delta p x-2 k \cos (x) .
$$

The potential $V(x)$ is illustrated in Figure 5.3 . For $\Delta p<2 k$ local minima of different depth exist as illustrated in Figure $5.3(\mathrm{a}, \mathrm{b})$. The larger the difference between $\Delta p$ and $2 k$ is the deeper is the minimum and thus the more difficult it is for the system to overcome the potential barrier. The system has thus a larger tolerance against perturbations for deeper minima in the sense that the system is still capable of reaching its stable state. The marginal stable case of $\Delta p=2 k$ is illustrated in Figure 5.3(c). As illustrated in Figure 5.3 (d) if $\Delta p$ is larger than $2 k$, no minimum exists and the system does not reach a stable state. The system is in this case in the globally unstable region of parameter space.

The phase difference of two oscillators cannot be larger than $2 \pi$. Therefore we can confine the potential $V(x)$ to the interval $[0,2 \pi)$. Because all minima are equivalent, there is no information lost via this confinement. The local minimum $V\left(x_{\min }\right)$ of the potential is now found at

$$
x_{\min }=\arcsin \left(\frac{\Delta p}{2 k}\right) \quad \text { for } \quad \Delta p<2 k .
$$

Reaching the minimum of the potential is equivalent to reaching the stable steady state 
of the original system. We have a constant phase difference between the two oscillators in the stable state. In addition a local maximum $V\left(x_{\max }\right)$ exists at

$$
x_{\max }=\pi-\arcsin \left(\frac{\Delta p}{2 k}\right) .
$$

The local maximum is equivalent to the unstable fixed point $T_{2}$ (cf. section 5.2). If the particle crosses the potential barrier for any reason, it depends crucially on the system parameters whether it does relax back to a steady state. The particle gains kinetic energy because of the tilting of the potential which is proportional to $\Delta p$. However, it also loses kinetic energy due to friction. In the globally stable regime of parameter space friction is so strong that the kinetic energy always decreases. The particle finally comes to rest. In the coexistence region a limit cycle exists where the average friction loses exactly matches the average increase of kinetic energy due to the tilting. The particle then converges to the limit cycle as soon as it crosses the potential barrier.

\subsection{Imbalanced power production and consumption}

As argued in the previous sections, power grids generally operate in the coexistence region of parameter space. For imbalanced power generation and consumption, i.e., $\left|P_{1}\right| \neq\left|P_{2}\right|$, the system does not exhibit a stable fixed point. The fixed points exist again as soon as equal power generation and production is restored. Whether the system does or does not relax back to the steady state when the power balance is restored or not, depends on the strength of the perturbation, which drives the system away from the steady state. We calculate the sum of both equations (5.1) and solve for the mean frequency

$$
\frac{\dot{\theta}_{1}+\dot{\theta}_{2}}{2}=-\frac{1}{\alpha}\left(\frac{P_{1}+P_{2}}{2}-\frac{\ddot{\theta}_{1}+\ddot{\theta}_{2}}{2}\right) .
$$

During stable operation we find $P_{1}+P_{2}=0$ and the phases are locked with $\dot{\theta}_{1}=\dot{\theta}_{2}=0$ and $\ddot{\theta}_{1}=\ddot{\theta}_{2}=0$ (cf. section 5.1). The mean frequency is a measure for the deviation from the grid frequency $\Omega$. The system thus runs with the grid frequency in the stable state.

If the system has imbalanced power production and consumption, no steady state with $\dot{\theta}_{1}=\dot{\theta}_{2}=0$ exists. Still generator and consumer can remain phase-locked such that a steady power flow is guaranteed. Phase-locking implies that $\theta_{1}-\theta_{2}=$ const, such that $\dot{\theta}_{1}=\dot{\theta}_{2}=$ const. The second derivatives on the right hand side of equation (5.35) still vanish, but we have constant values for the first derivatives. With $\ddot{\theta}_{1}=\ddot{\theta}_{2}=0$ we thus have

$$
\frac{\dot{\theta}_{1}+\dot{\theta}_{2}}{2}=-\frac{P_{1}+P_{2}}{2 \alpha}
$$

Two examples for such a relaxation are illustrated in Figure 5.4(a,b) for weak and in 


\section{Dynamics \& self-organization of an elementary model}

(a)

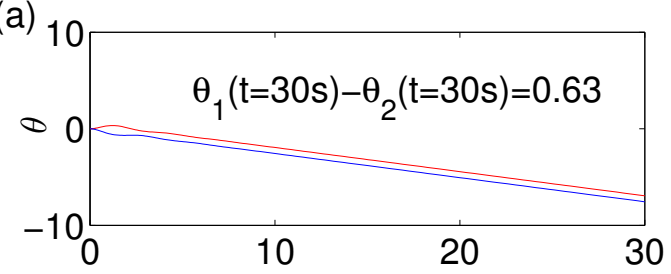

(b)

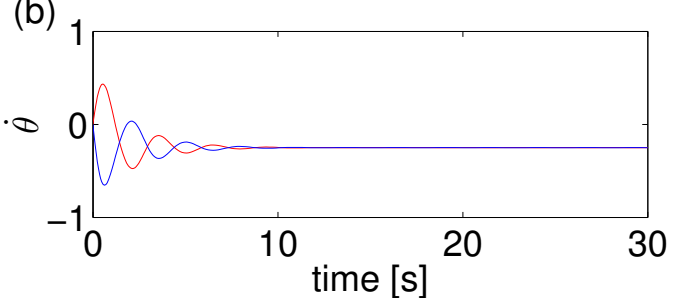

(c) 50

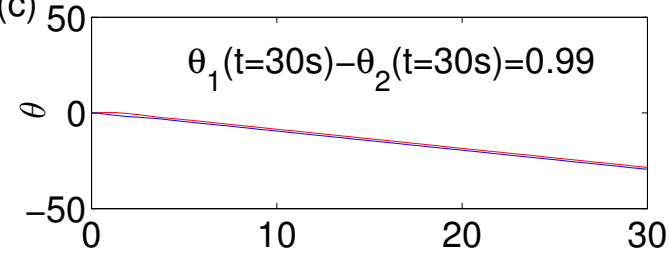

(d)

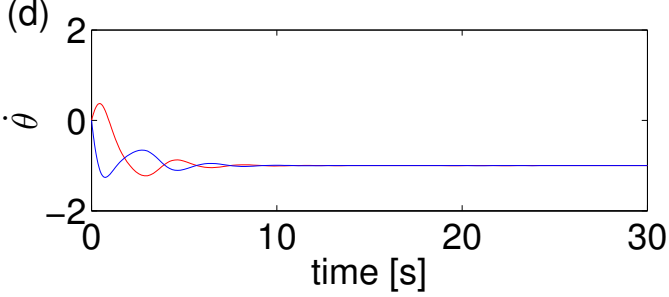

Figure 5.4: Imbalanced power production and consumption. Parameters are: $\alpha=1 \mathrm{~s}^{-1}, K=$ $3 \mathrm{~s}^{-2}$ and $P_{1}=1.5 \mathrm{~s}^{-2}$. red: generator $\left(\theta_{1}\right.$ and $\left.\dot{\theta}_{1}\right)$, blue: consumer $\left(\theta_{2}\right.$ and $\left.\dot{\theta}_{2}\right)$. (a,b) Consumer with a slightly higher demand: $P_{2}=-2 \mathrm{~s}^{-2}$. (c,d) Consumer with a strongly higher demand: $P_{2}=-3 \mathrm{~s}^{-2}$. The phases of generator and consumer are locked with constant phase derivatives. The difference between the phases and the phase derivatives are larger for stronger imbalances between power production and consumption.

Figure $5.4(\mathrm{c}, \mathrm{d})$ for strong inequalities of power production and consumption. For both inequalities we have a deviation from the desired grid frequency $\Omega$ for both the generator and the consumer. The deviations increase with the strength of the inequality between power production and consumption as illustrated in Figure 5.4 (b,d). However, both elements have the same deviation regardless of weak or strong inequalities, such that they are still phase-locked. The phase difference $\theta_{1}-\theta_{2}$ is constant. The phase difference between the elements increases with increasing deviations from the grid frequency as illustrated in Figure 5.4(a,c). Thus the difference between the phase derivatives $\Delta \chi=\theta_{1}-\theta_{2}$ is always zero, but the difference between the phases $\Delta \theta$ increases with growing deviations. As it is illustrated in Figure $5.2(\mathrm{~h}, \mathrm{i})$ the system cannot reach the stable fixed point again if the difference between the phases is too large. In order to avoid this danger, there is a boundary for tolerable deviations from the grid frequency implemented for the operation of real-world power grids. This boundary for tolerable deviations from the grid frequency is $\pm 0.2 \mathrm{~Hz}$ [25]. If larger deviations occur countermeasures are executed [25].

\subsection{Self-organized adaptation}

If the condition of equal power generation and consumption is violated, the oscillators may still synchronize, but at a different value than the grid's reference frequency. As shown in the preceding section, if the generated power exceeds the consumed power the oscillators accelerate until the excess power is balanced by increased friction losses. This is strongly 

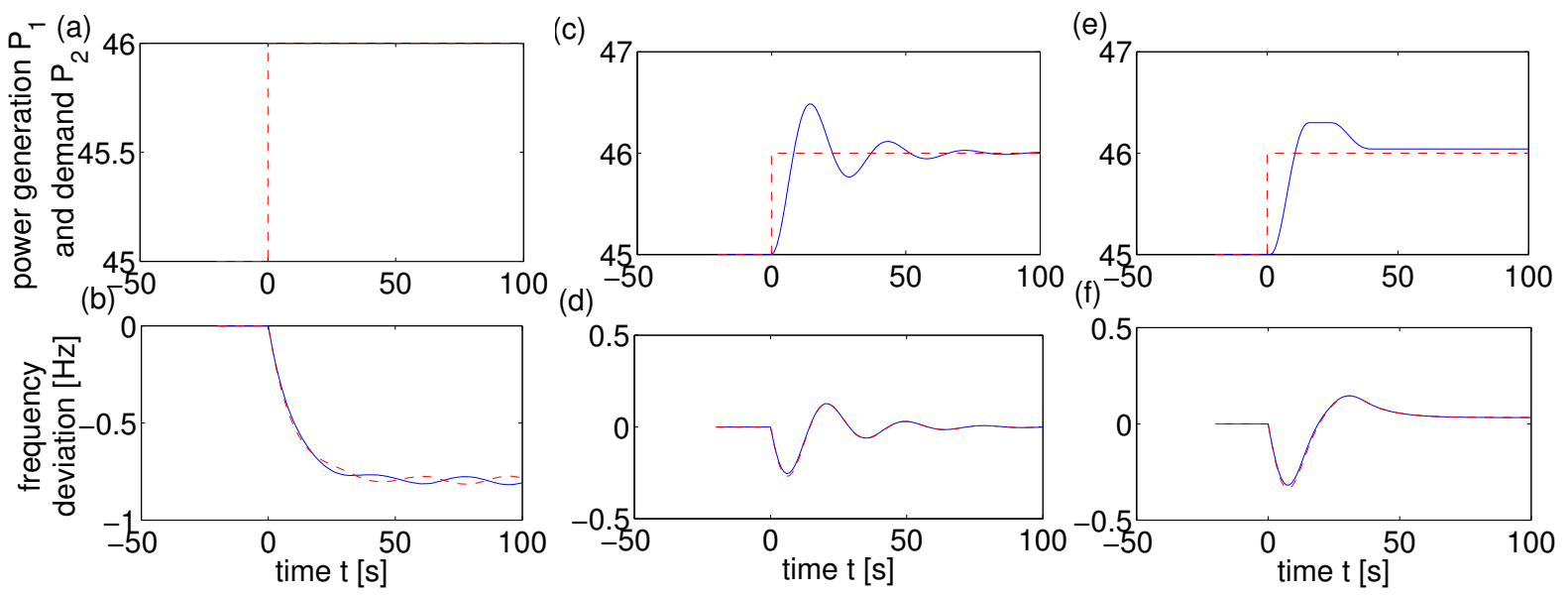

Figure 5.5: Adaptation principles. The upper panels (a,c,e) show the power generation $P_{1}$ (solid blue line) and the demand $P_{2}$ (dashed red line), the lower panels (b,d,f) show the deviations of $\dot{\theta}_{1} / 2 \pi$ (blue) and $\dot{\theta}_{2} / 2 \pi$ (red) from the grid reference frequency $\Omega / 2 \pi$. Parameters: $\alpha=0.1 \mathrm{~s}^{-1}$, $P_{1}=45 \mathrm{~s}^{-2}, P_{2}=-45 \mathrm{~s}^{-2}, K=50 \mathrm{~s}^{-2}$. At $t=0$ the power demand is increased to $P_{2}=-46 \mathrm{~s}^{-2}$. $(\mathrm{a}, \mathrm{b})$ Without any adaptation process the system relaxes to a phase-locked state, but the local frequency deviates significantly from the grid reference frequency. (c,d) The local frequency adapts to the grid frequency and the produced power $P_{1}$ adapts proportional to the generator's deviation from the grid frequency with $\dot{P}_{1}=-c \dot{\theta}_{1}$ with $c=0.1 \mathrm{~s}^{-2}$. (e,f) Same adaptation process as in (c,d) which sets in whenever the deviation from $\Omega$ exceeds the value $\left|\dot{\theta}_{1}\right|=2 \pi \cdot 0.1 \mathrm{~Hz}$. The grid stabilizes with less oscillations but a remaining offset.

undesirable from a technical viewpoint, because many important electrical machines are optimized for a fixed grid frequency. Therefore the grid has to be controlled to guarantee frequency stability. Currently, this is achieved by the so-called primary control in power plants 22 . In primary control, the grid frequency is constantly measured. If deviations from the grid reference frequency are detected, the produced power needs to be adapted.

Here we demonstrate different principles of adapting the produced power in a power grid. We assume that the power grid is initially in its stable steady state. At $t=0$ we increase the consumed power from $P_{2}=-45 \mathrm{~s}^{-2}$ to $P_{2}=-46 \mathrm{~s}^{-2}$. The frequency dynamics that results without adaption is illustrated in Figure $5.5(\mathrm{a}, \mathrm{b})$. The system relaxes to a synchronous state at a frequency $0.8 \mathrm{~Hz}$ below the reference frequency $\Omega / 2 \pi$, a deviation which is significantly larger than allowed by current stability regulation [25]. If such an event occurs the grid operater has to carry out immediate countermeasures up to an emergency shutdown to prevent large scale outages.

To prevent such an event, the produced power has to be adapted according to the demand. The dynamics of two different adaption strategies are illustrated in Figure 5.5(c,d,e,f). In panels $(\mathrm{c}, \mathrm{d})$ we assume that adaptation of the produced power sets in as soon as deviations 


\section{Dynamics \& self-organization of an elementary model}

from the grid frequency occur. The produced power adapts according to

$$
\dot{P}_{1}(t)=-c \dot{\theta}_{1}
$$

with $c=0.1 \mathrm{~s}^{-2}$. The system reaches balanced power production and consumption and runs with the exact desired grid frequency.

In panels $(e, f)$ power adaptation only sets in whenever the deviations are larger than a tolerance of $2 \pi \cdot 0.1 \mathrm{~Hz}$. The produced power adapts according to

$$
\dot{P}_{1}= \begin{cases}-c\left(\dot{\theta}_{1}-2 \pi \cdot 0.1 \mathrm{~Hz}\right) & \text { for } \dot{\theta}_{1}>2 \pi \cdot 0.1 \mathrm{~Hz} \\ -c\left(\dot{\theta}_{1}+2 \pi \cdot 0.1 \mathrm{~Hz}\right) & \text { for } \dot{\theta}_{1}<-2 \pi \cdot 0.1 \mathrm{~Hz} \\ 0 & \text { otherwise }\end{cases}
$$

with $c=0.1 \mathrm{~s}^{-2}$. The relaxation is faster if a band of tolerable deviations is employed, but a small tolerable frequency deviation remains.

\subsection{Discussion}

In summary, we conducted a detailed analysis of a simple power grid consisting of one generator and one consumer. We derived the effective equations of motion of the power grid and carried out a detailed stability analysis. We identified three different stability regimes. A globally unstable regime with a limit cycle where no steady state exists, a globally stable regime, where the dynamics converge to the limit cycle for all initial conditions and a coexistence regime, where both the stable fixed point and the limit cycle exist. This regime is the most interesting one, because here the dynamics crucially depend on the initial conditions. We provided an analytical approximation for the border in parameter space between the globally stable and the coexistence regime. Furthermore, we demonstrated that the dynamics of the power grid is equivalent to the dynamics of a particle in a tilted washboard potential. We showed that the motion of the particle also converges either to a resting state, i.e., to the stable fixed point or to a limit cycle. We continued with an analysis of a state with imbalanced power production and consumption. Here the stable fixed point does not exist. Depending on the strength of the imbalance the power grid then operates at a different frequency, which deviates from the desired grid frequency. Finally, we demonstrated different adaptation principles for power production. We showed that the inclusion of small tolerable deviations from the grid frequency allows for a faster power adaptation process.

It is important to note, that the existence of the coexistence regime is one of the most important features of real-world power grids. Most major power grids are operating close to the edge of stability, i.e., in the region of coexistence, at least during periods of high loads. Therefore the dynamics depends crucially on the initial conditions and static power 
grid models are insufficient. The oscillator power model captures this important feature. It thus allows the extensive study of the decentralization process in the next chapter, because it is a priori not clear if the dynamics converge to the fixed point or the limit cycle. We proceed with a study of the synchronization transition and robustness against dynamic perturbations of larger power grids in the next chapter. 



\section{Decentralized power generation in future power grids}

In this chapter we investigate, how decentralizing power generation changes the collective dynamical features of power grids. The decentralization process itself takes place due to the inclusion of more and more renewable energy sources into the grid as a replacement for conventional power sources. Decentralization is an ongoing process in real world power grids. It is even called the "new power paradigm for the next millennium by Borbely and Kreider [17]. The International Energy Agency (IEA) lists a number of reasons for the decentralization process [61], which are in short: new developments in distributed generation technologies, constraints in the construction of new transmission lines, increased customer demand, electricity market liberalization and climate concerns 62 .

The decentralization process will alter the topology of the existing power grid in a yet unknown way as power production will become more recurrent [18]. However, the general impact of grid topologies on collective dynamics is not systematically understood, in particular with respect to decentralization. It is for instance still an open question if the addition of new transmission lines into power grids decreases their vulnerability against outages of individual elements [61, 63] or opens the door to the possibility of cascading failures [64]. In the following we analyze the effects of the decentralization process on the capability of power grids of maintaining stable operation for three different general topologies (regular, random and small-world) and the special topology of the British power grid. With regard to our model, maintaining stable operation is equivalent to maintaining a phase-locked state (cf. section 5.2) where all elements oscillate with the same frequency.

Furthermore, we analyze the structural stability of power grids. We determine if a power grid can maintain stable operation if one transmission line fails and count the number of critical transmission lines that are indispensable for the operation of the grid, as a global measure of network stability. This resembles the N-1 safety criterion [24], which is defined as follows: No transmission line may become overloaded, i.e., needs to carry a load bigger than its transmission capacity if any other transmission line fails [24]. This criterion has to be fulfilled for the European power grid by power grid regulations [23]. For the power outage in Western Europe in November 2006 this criterion was violated [18. The

Parts of this chapter have been previously published in 19,58 . 


\section{Decentralized power generation in future power grids}

structural stability is thus a global measure of the amount of critical transmission lines, i.e., transmission lines that would cause power outages if they failed.

In the first section we introduce four model networks with different topological properties and the decentralization process for each of them. In the next section we demonstrate that the onset of phase-locking in dependance of the parameters of the grid, such as the capacity of transmission lines, is promoted for higher degrees of decentralization. We proceed with an analysis of the synchronization time, i.e., the time needed to reach the stable state, in dependance of the degree of decentralization. We show that decentralization has no negative effect on the speed of synchronization. Furthermore, we analyze the robustness of power grids against dynamical perturbations as for example an increase in power demand by the consumers. We demonstrate that centralized grids generally have a higher robustness against such perturbations. Finally, we show that structural stability, e.g. the tolerance against a breakdown of a transmission line, is increased for a higher degree of decentralization. The chapter concludes with a brief summary and discussion.

\subsection{Modeling the decentralization process}

In this section we introduce the different classes of model networks analyzed in the following. We consider four different topology classes: three established network ensembles and the topology of the British power grid as an example of a real-world power grid. As examples of network ensembles, we consider quasi-regular grids, Erdős-Rényi random graphs [65, 66] and Watts-Strogatz small-world models [67]. For each network we define a different decentralization process for power production. Generally, the conventional power sources are removed from the grid and replaced by small decentralized power sources located all over the grid. We explain the details of the decentralization process in the following.

\subsubsection{Modeling the decentralization process of random network ensembles}

Sketches of a quasi-regular grid, an Erdös-Rényi random graph [65, 66] and a WattsStrogatz small-world grid [67] are illustrated in Figure 6.1. In the following we consider power grids of $N_{C}=100$ consumer units with the same power load $-P_{0}$ each. In all simulations we assume $P_{0}=1 \mathrm{~s}^{-2}$ and $\alpha=0.1 \mathrm{~s}^{-1}$ as discussed in section 4.2.5. We distinguish between large centralized power plants and small decentralized ones. The large power plants produce a power of $P_{P}=10 P_{0}$ each, the small decentralized sources produce $P_{R}=2.5 P_{0}$ each (cf. section 4.2.5). The amount of centralized power sources $N_{P}$ varies from $N_{P} \in\{0, \ldots, 10\}$, the amount of small decentralized power sources $N_{R}$ complements the large power sources in such a way, that the total demand is matched. For instance, in 
(a)

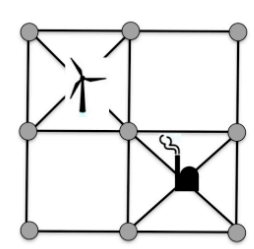

(b)

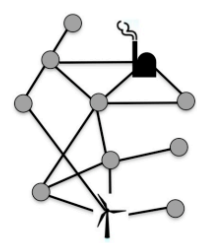

(c)

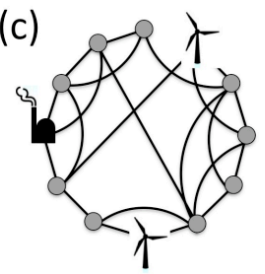

Figure 6.1: Small size cartoons of different network topologies: (a) Quasi-regular grid, (b) random network and (c) small-world network.

a network with $N_{P}=5$ centralized power plants and $N_{R}=5 \cdot 4=20$ decentralized sources, $50 \%$ of the total power is produced by decentralized power sources.

The quasi-regular network illustrated in Figure 6.1(a) consists of a square lattice, where every node has four connections to its nearest neighbors on the square lattice. The power plants are randomly located in the middle of the squares of the lattice. They are connected to its four nearest neighbors as depicted. The decentralization process is simulated as follows. We start with $N_{P}=10$ and $N_{R}=0$, i.e., the power is entirely produced by large centralized power plants. We remove one of these generators from the grid and replace it by four small generators, which are placed randomly onto free squares of the grid. This procedure continues until no large power plants remains in the grid.

Next we define the decentralization process for an Erdős-Rényi random graph where every node has an average of six completely random connections to the other nodes. Networks which are not connected are discarded. The decentralization process works as described above. We remove one of the large power plants from the grid and add four small power sources into the grid, connecting them with an average of six random connections into the grid. A sketch of an Erdős-Rényi random graph with an average of three connections is illustrated in Figure 6.1(b).

A sketch of a Watts-Strogatz small-world network with four connections is illustrated in Figure 6.1(c). The small-world network we consider is obtained by a standard rewiring algorithm as described in [67]. Starting from a regular ring network, where every node $i$ is connected to its four nearest neighbors, each connection $i j$ between nodes $i$ and $j$ is randomly rewired with a probability of 0.1. If a connection $i j$ starting from node $i$ is rewired to a different node $k$ with $k \neq j$, the node $k$ is chosen from an uniform probability distribution of all nodes that avoids self-loops and link duplications. The decentralization process is the same as described above. We remove one of the large centralized power sources from the grid and replace it by four small generators. The small generators are 


\section{Decentralized power generation in future power grids}

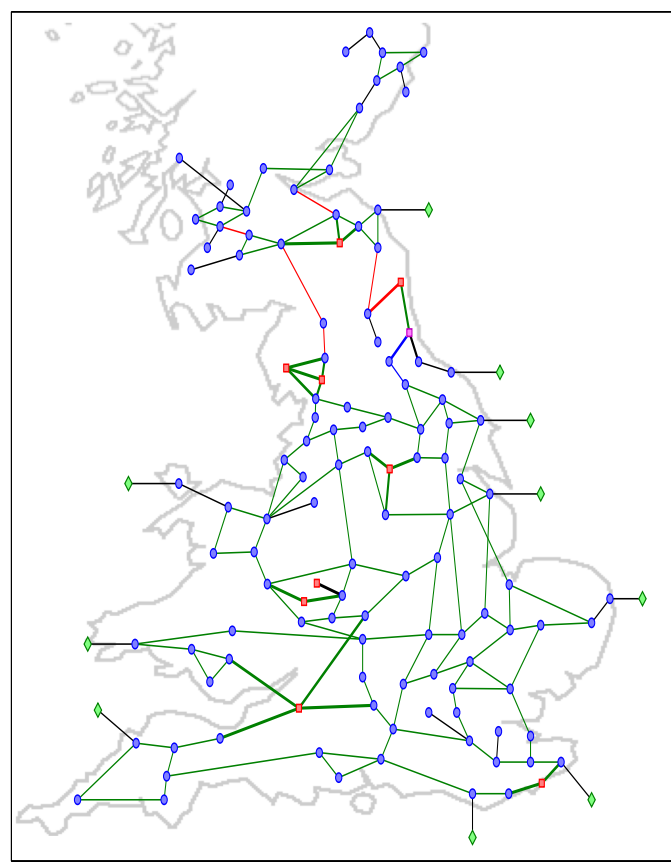

Figure 6.2: The Figure illustrates the simulated decentralization of the British grid, replacing large centralized power plants by small distributed ones. First step of decentralizing: one power plant (marked by violet square) is disabled, i.e., the generated power is set to zero $\left(P_{j}=\right.$ $0)$. Instead, ten new small generators are added to the grid at random positions (green diamonds). For clarity the Figure demonstrates an example where the small decentralized generators are only added to the grid at outside positions.

connected into the grid with the same rewiring process.

\subsubsection{Modeling the decentralization process of the British grid}

The coarse grained structure of the British power grid is illustrated in Figure 6.2. The British high-voltage power grid consists of 120 nodes and 165 transmission lines [37]. Initially, ten nodes are randomly selected to be centralized power plants, the others are consumers. Power plants are connected to their neighbors with a higher capacity $c K$ with $c \geq 1$. The remaining transmission lines have a capacity $K$. Each consumer has the same power load $-P_{0}$ with $P_{0}=1 \mathrm{~s}^{-2}$ as described above. We have $\alpha=0.1 \mathrm{~s}^{-1}$ for all simulations (cf. section 4.2.5). To further strengthen the effects of decentralization we choose slightly different parameters of $P_{P}=11 P_{0}$ and $P_{R}=1.1 P_{0}$ for the power outputs of large centralized and small decentralized power sources, respectively. Large power sources are thus replaced by ten smaller ones.

The decentralization process works as follows. The output of the large centralized power plants are set to zero one after another and new decentralized power sources are connected by one new transmission to a randomly chosen node of the existing grid. This process continues until no centralized power source is left. An example for the first step of the decentralization process for the British power grid is illustrated in Figure 6.2. One large centralized power plant marked by the violet square is disabled, i.e., its power output is set to zero. After that ten new small decentralized power sources marked by green diamonds are added into the grid. This process continues until all large power plants are replaced by 
small decentralized power plants and we thus have $100 \%$ power generation by renewable power plants.

\subsection{The synchronization transition}

Synchronization of Kuramoto oscillators is defined as the state, where all oscillators run with same frequency and have the same phase [68. In our considered model, stable operation of a power grid requires only that all machines run with the same frequency. The phases of the machines are generally different, but the phase differences are constant in time in stable operation (cf. section 5.3). Stable operation of a power grid thus requires a phase-locked state. However, because the phases of the machines are generally different such a state is not synchronized in the Kuramoto sense. The degree of phase-locking can be measured by the order parameter (see below) or the so called phase cohesiveness [51]. The phase cohesiveness gives an upper bound to the phase differences between adjacent nodes in the networks. If all machines of the grid run with the same frequency the system is thus always phase cohesive. If the machine do not all run with the same frequency the system is not phase cohesive.

The phase-locked state must be distinguished from the partly synchronous state commonly analyzed in the context of the Kuramoto model [68]. Here, a fraction of the Kuramoto oscillators remain incoherent. Such a state has a non-vanishing order parameter but is not phase-cohesive.

The capability of a power grid to reach a stable state depends on the power grid's parameters. In this section we analyze the synchronization transition, i.e., the minimal required transmission capacity $K_{c}$ for the onset of phase-locking between generators and consumers. At first we calculate $K_{c}$ for simple motif networks that serve as building blocks for the larger networks. The obtained results for these analytically tractable motif networks can be regarded as lower bounds for the results of our for different larger network topologies [68]. If the transmission capacity $K$ exceeds $K_{c}$ the system is either in the globally stable or the coexistence regime of parameter space. The boundary between these two regimes can only be determined numerically. However, we can generally say that if $K$ is still close to $K_{c}$, the system is in the coexistence regime. It is globally stable only for $K \gg K_{c}$. If $K$ is below $K_{c}$ the system is unstable.

\subsubsection{Synchronization transition of motif networks}

The two motif networks are illustrated in Figure 6.3. They can be considered as building blocks of the large-scale quasi-regular network that will be analyzed in the next chapter. A simple network, where a small renewable energy source provides the power for $N=3$ consumer units with $d=3$ connections, is illustrated in Figure 6.3(a). To analyze the 


\section{Decentralized power generation in future power grids}
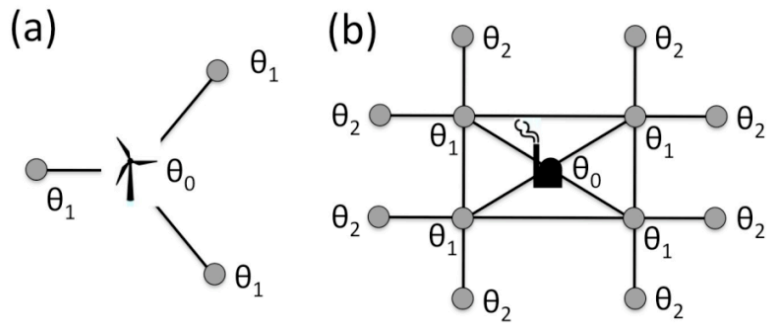

Figure 6.3: Two motif networks with simplified phase description.

most homogeneous setting we assume that all consumers have the same phase $\theta_{1}$ and a power load of $-P_{0}$ and that all transmission lines have the same capacity $K$. The power generator has the phase $\theta_{0}$ and provides a power of $N P_{0}$. The reduced equations of motion then read

$$
\begin{aligned}
& \ddot{\theta}_{0}=N P_{0}-\alpha \dot{\theta}_{0}+d K \sin \left(\theta_{1}-\theta_{0}\right), \\
& \ddot{\theta}_{1}=-P_{0}-\alpha \dot{\theta}_{1}+K \sin \left(\theta_{0}-\theta_{1}\right) .
\end{aligned}
$$

For this motif class the condition $|N|=|d|$ always holds, such that the steady state is determined by $\sin \left(\theta_{0}-\theta_{1}\right)=P_{0} / K$. The condition for the existence of a steady state is thus (cf. section 5.2)

$$
K>K_{c}=P_{0},
$$

i.e., each transmission line must be capable of transmitting the power load of one consumer unit.

As a second motif, we analyze a part of the quasi-regular grid with $N=12$ consumer units. The motif is illustrated in Figure 6.3 (b). The central power source with phase $\theta_{0}$ and the nearest consumers with phase $\theta_{1}$ have $d_{1}=4$ connections. The consumers with phase $\theta_{1}$ and those with phase $\theta_{2}$ have $d_{2}=2$ connections. Due to the symmetry of the system we have to consider only three different phases. The reduced equations of motion then read

$$
\begin{aligned}
& \ddot{\theta}_{0}=N P_{0}-\alpha \dot{\theta}_{0}+d_{1} K \sin \left(\theta_{1}-\theta_{0}\right), \\
& \ddot{\theta}_{1}=-P_{0}-\alpha \dot{\theta}_{1}+d_{2} K \sin \left(\theta_{2}-\theta_{1}\right)+K \sin \left(\theta_{0}-\theta_{1}\right), \\
& \ddot{\theta}_{2}=-P_{0}-\alpha \dot{\theta}_{2}+K \sin \left(\theta_{1}-\theta_{2}\right) .
\end{aligned}
$$

For the steady state we thus find the relations

$$
\begin{aligned}
& \sin \left(\theta_{0}-\theta_{1}\right)=\left(N P_{0}\right) /\left(d_{1} K\right), \\
& \sin \left(\theta_{1}-\theta_{2}\right)=P_{0} / K .
\end{aligned}
$$


The transmission capacity $K$ must now be higher than the critical coupling strength

$$
K_{c}=\frac{N P_{0}}{d_{1}}
$$

to enable a stable operation. For the example motif we thus have a higher critical coupling strength of $K_{c}=3 P_{0}$ compared to the previous motif for the existence of a steady state. This is immediately clear from physical reasons, as the transmission lines leading away from the power plant now have to serve three consumer units instead of just one.

The study of motif networks reveals one immediate mechanism of how decentralization affects the synchronization transition in power grids. However, there is also a significant effect beyond this rather obvious mechanism as we will show in the following. To reveal these more subtle effects, we increase the transmission capacity of transmission lines incident to a large power by a factor of $c \geq 1$ in the study of the British power grid (cf. section 6.2.3.

\subsubsection{Synchronization transition of random model networks}

For the large model networks of random, small-world and regular grids we introduce the order parameter $r(t)$ to quantify the degree of synchronization. The order parameter 30] is now defined as

$$
r(t)=\frac{1}{N} \sum_{j=1}^{N} e^{i \theta_{j}(t)} .
$$

If the system is fully synchronized, i.e., all phases are equal, the real part of the order parameter is $\Re(r(t))=1$. In a phase-locked state the real part of the order parameter $r(t)$ can in general be any positive value between zero and one. Here $\Re(r(t))$ is in fact close to one for all of our model networks if the system is in a phase-locked state. If the system does not reach a phase-locked state, the real part of the order parameter fluctuates around zero.

An example for the relation between the phases of the machines and the order parameter is illustrated in Figure 6.4. The dynamics of the phases $\theta_{j}(t)$ of all machines $j$ and the real part of the order parameter are shown for two different values of the coupling strength $K$. Without coupling, $K=0$, all elements of the grid oscillate with their natural frequency $\omega$. For small values of $K$ with $K<K_{c}$, only the phases of the decentralized generators and the consumers are close together as illustrated in Figure 6.4(a). The system is thus not in its stable state and the real part of the order parameter fluctuates around zero. If the coupling strength is further increased, as illustrated in Figure 6.4(b), such that we have $K>K_{c}$, all generators phase-lock as well, such that a stable operation of the power grid is possible. Consequently, the real part of the order parameter is close to one.

In the long time limit, the system will either relax to a steady phase-locked state or to 


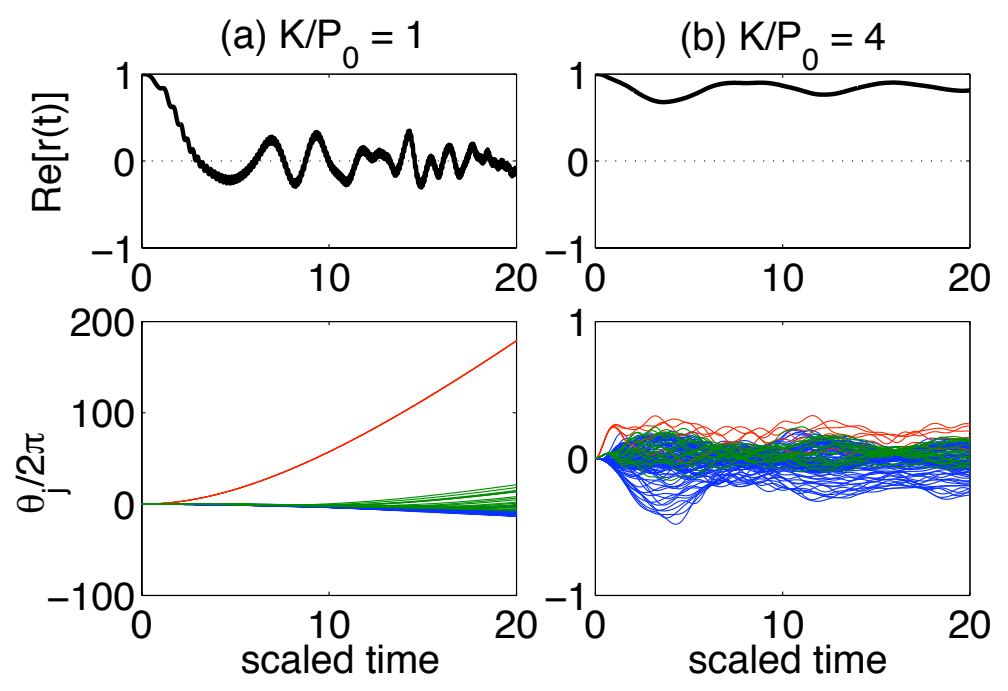

Figure 6.4: Phase dynamics of a quasi-regular power grid. (a) For weak coupling the phases $\theta_{j}(t)$ of the small renewable decentralized generators (green lines) are close to the consumer's phases (blue lines), but not the phases of the large power plants (red lines). Thus the order parameter $r(t)$ fluctuates around a zero mean. (b) Global phase-locking of all generators and consumers is achieved for a large coupling strength, such that the real part of the order parameters $r(t)$ has a positive value (here close to one).

a limit cycle where the generators and consumers are decoupled and $\Re(r(t))$ oscillates around zero. In order to quantify synchronization in the long time limit we thus define the averaged order parameter

$$
r_{\infty}:=\lim _{t_{1} \rightarrow \infty} \lim _{t_{2} \rightarrow \infty} \frac{1}{t_{2}} \int_{t_{1}}^{t_{1}+t_{2}} r(t) d t
$$

In numerical simulations the integration time $t_{2}$ must be finite, but large compared to the oscillation period if the system converges to a limit cycle. Furthermore, we consider the averaged squared phase velocity

$$
v^{2}(t)=\frac{1}{N} \sum_{j=1}^{N} \dot{\theta}_{j}(t)^{2}
$$

and its limiting value

$$
v_{\infty}^{2}:=\lim _{t_{1} \rightarrow \infty} \lim _{t_{2} \rightarrow \infty} \frac{1}{t_{2}} \int_{t_{1}}^{t_{1}+t_{2}} v^{2}(t) d t
$$

as a measure of whether the grid relaxes to a steady state or not. In the steady state we have $v_{\infty}=0$ because all phase derivatives are zero (cf. section 5.2). If we have $v_{\infty} \neq 0$, the system is not in its stable state. The two quantities $r_{\infty}$ and $v_{\infty}$ are plotted in Figure 6.5 as a function of the coupling strength $K / P_{0}$ for 20 realizations of a quasi-regular network with $N_{C}=100$ consumers and $40 \%$ renewable energy sources. The onset of phase-locking is 

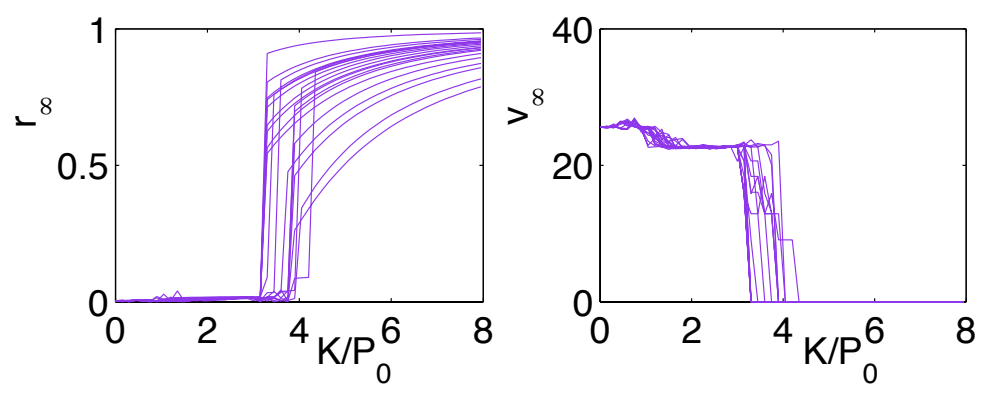

Figure 6.5: The synchronization transition as a function of the coupling strength $K$ : The order parameter $r_{\infty}$ (left-hand side) and the phase velocity $v_{\infty}$ (right-hand side) in the long time limit. The dynamics has been simulated for 20 different realizations of a quasi-regular network consisting of 100 consumers, $N_{P}=6$ large power pants and $N_{R}=16$ small power generators.

clearly visible: If the transmission capacity is smaller than the critical value $K_{c}$, no steady phase-locked state exists and we have $r_{\infty}=0$. Increasing $K$ above $K_{c}$ leads to the onset of phase-locking such that $r_{\infty}$ jumps to a non-zero value. The critical value of the coupling strength is found to lie in the range $K_{c} / P_{0} \approx 3.1-4.2$ where $v_{\infty}$ reaches zero. The critical value depends on the random realization of the network topology.

The synchronization transition is quantitatively analyzed for the three different general network topologies in Figure 6.6. We plotted $r_{\infty}$ and $v_{\infty}$ averaged over 100 random realizations for each amount of decentralized energy sources and for every topology. The synchronization transition strongly depends on the structure of the network and in particular the amount of power provided by small decentralized energy sources. Each line in Figure 6.4 corresponds to a different fraction of decentralized energy $1-N_{P} / 10$, where $N_{P}$ is the number of large conventional power plants feeding the grid. Most interestingly, the introduction of small decentralized power sources (i.e. the reduction of $N_{P}$ ) promotes the onset of phase-locking. The onset of phase-locking is most obvious for the random and the small-world structures as illustrated in Figure 6.6(a,b).

Let us analyze the quasi-regular grid in the limiting cases $N_{P}=10$ (only large power plants) and $N_{P}=0$ (only small decentralized power stations) in detail. The existence of a phase-locked steady state requires that the transmission lines leading away from a generator have enough capacity to transfer the whole power, i.e., $10 P_{0}$ for a large power plant and $2.5 P_{0}$ for a small power station. In a quasi-regular grid every generator is connected with exactly four transmission lines, which leads to the following estimate for the critical coupling strength (cf. (6.5)):

$$
\begin{array}{ll}
K_{c}=10 P_{0} / 4 & \text { for } N_{P}=10, \\
K_{c}=2.5 P_{0} / 4 & \text { for } N_{P}=0 .
\end{array}
$$

These values only hold for a completely homogeneous distribution of the power load and thus rather present a lower bound for $K_{c}$ in a random network realization. Indeed, the 


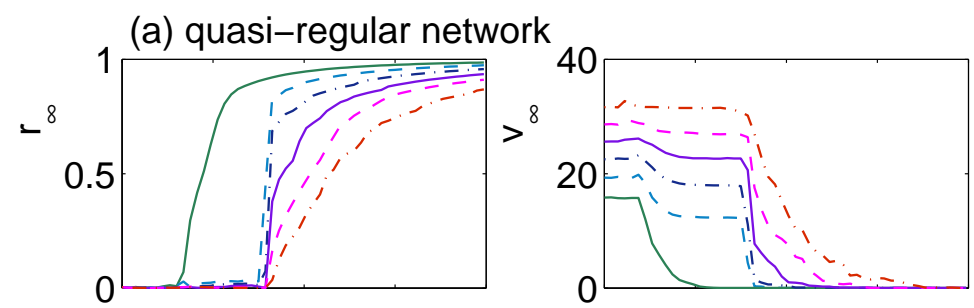

(b) random network
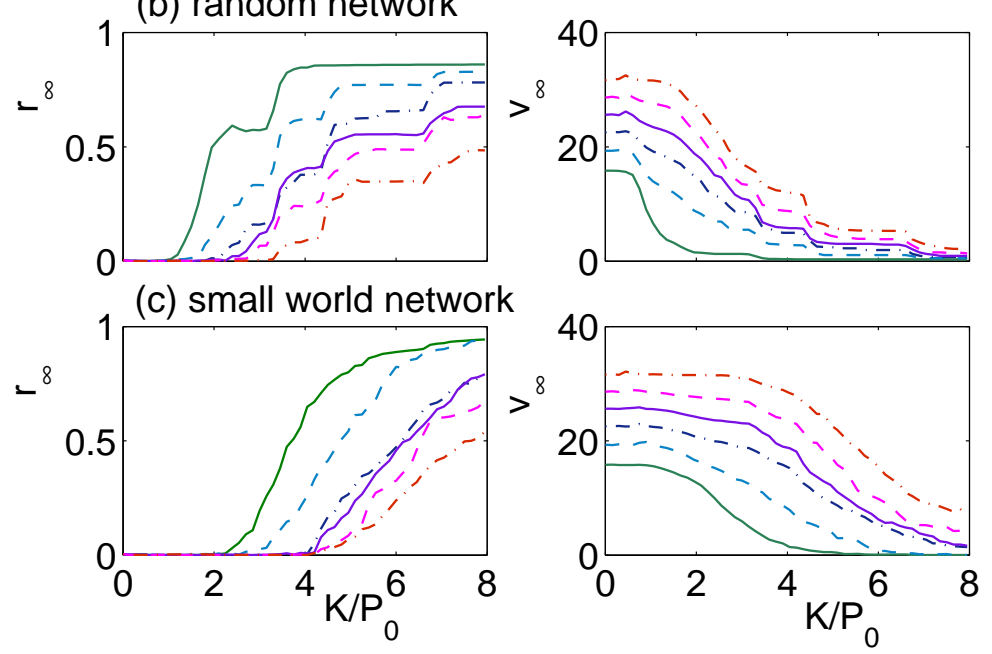

Fraction of distributed energy sources [\%]:

$$
-100--80 \cdots 60-40---20 \cdots 0
$$

Figure 6.6: The synchronization transition for different fractions of decentralized energy sources $1-N_{P} / 10$ feeding the grid and for different network topologies: (a) Quasi-regular grid, (b) random network and (c) small-world network. The order parameter $r_{\infty}$ and the phase velocity $v_{\infty}$ have been averaged over 100 realizations for each network structure and each fraction of decentralized sources.

numerical results illustrated in Figure 6.6(a) yield a critical coupling strength of $K_{c} \approx$ $3.2 \times P_{0}$ and $K_{c} \approx 1 \times P_{0}$, respectively (cf. (6.5) and (6.2)). However, the motifs provide only rough estimates and may serve as lower bounds for the actual synchronization transition because topological disorder typically increases the synchronization threshold 68.

\subsubsection{Synchronization transition of the British grid}

Generally, larger networks of complex topologies exhibit self-organized phase-locking as shown in the previous subsection. This holds true for the coarse-scale topology of the British power grid as well. For every realization of the British grid ten different nodes are randomly selected to be centralized power plants. The network of one realization of the coarse-scale topology of the British power grid 37] is illustrated in Figure 6.7(a). Transmission lines connecting the power plants into the grid have a factor $c$ higher transmission 


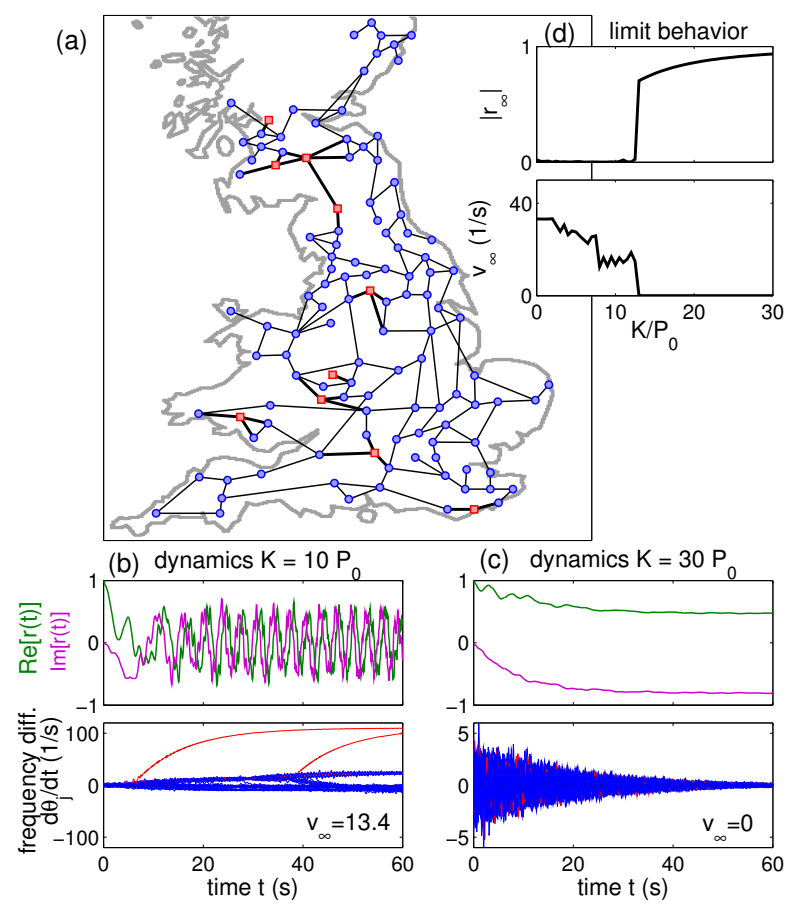

Figure 6.7: Synchronization transition to self-organized phase-locking in the British power grid. (a) Topology of the British power grid, consisting of 120 nodes and 165 transmission lines (black lines) [37. Ten nodes are randomly selected to be centralized power plants $\left(P_{j}=11 P_{0}, \square\right)$, the others are consumers $\left(P_{j}=-P_{0}, \circ\right)$. Power plants are connected to their neighbors with a higher capacity $c K, c \geq 1$ (thick lines), the remaining transmission lines have a capacity $K$ (thin lines). (b,c) Dynamics of the generators' (red) and consumers' (blue) frequencies $d \phi_{j} / d t$ and the respective order parameter $r(t)$ (b) for weak and (c) strong coupling. (d) The order parameter $r_{\infty}$ and the asymptotic mean frequency difference $v_{\infty}$ as a function of the coupling strength $K$ for $c=2$.

capacity.

There are two different possible scenarios. If the capacity of the transmission lines is below the critical value $K_{c}$, no steady state of the power grid exists. This is illustrated in Figure 6.7 (b). Consequently, the real part of the order parameter $r(t)$ fluctuates around zero in the upper panel. In the lower panel the phase derivatives of the generators and consumers are depicted. All machines develop large frequency deviations to the grid frequency $\omega$ over time. The generators accumulate energy such that their phases $\theta_{j}(t)$ are accelerated while the majority of consumers slow down. Notably, the generators do not desynchronize at once but rather in a cascade of failures (cf. [35, 37, 69]). Due to the damping, the system tends towards a limit cycle where the average phase velocity

$$
v(t)=\frac{1}{N} \sum_{j=1}^{N} \dot{\theta}_{j}(t)
$$




\section{Decentralized power generation in future power grids}
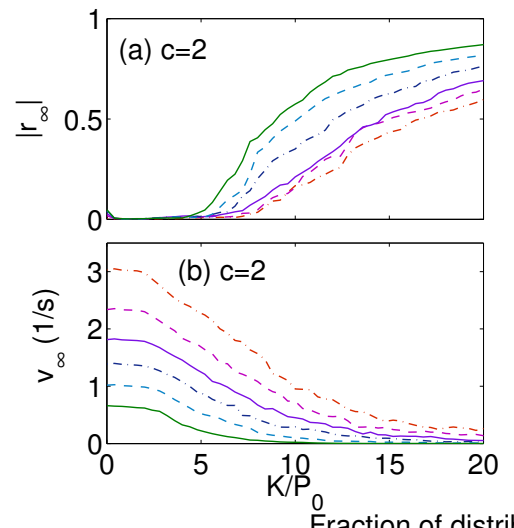

Fraction of distributed generators (\%):

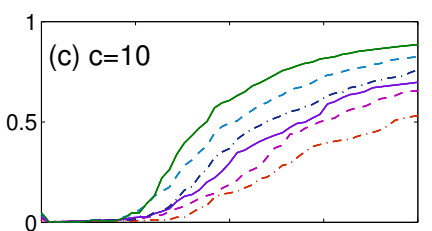

(d) $\mathrm{C}=10$

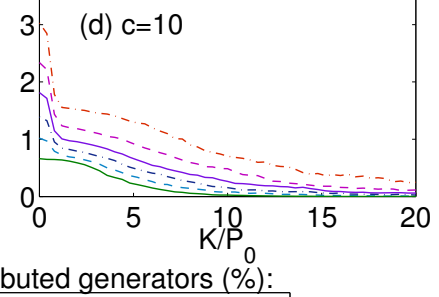

$0--20-40-60--80-100$

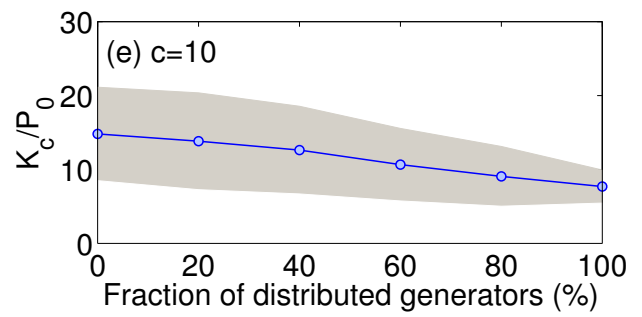

Figure 6.8: Promotion of self-organized phase-locking due to the replacement of centralized power plants. (a,b) Order parameter $\left|r_{\infty}\right|$ and the mean phase velocity $v_{\infty}$ as a function of coupling strength $K$ for $c=2$. (c,d) Order parameter and the mean phase velocity as a function of coupling strength for $c=10$. (e) Change of critical coupling strength $K_{c}$ for the onset of phaselocking due to the replacement of centralized power plants for $c=10$. Quantities are averaged over 100 realizations, as in Fig. 6.6. The shaded area shows the standard deviation.

and the mean phase velocity $v_{\infty}(\mathrm{b}, \mathrm{d})$

assumes a non-zero value in the long time limit:

$$
v_{\infty}>0
$$

If the capacity of the transmission lines is above $K_{c}$, all machines of the power grid phaselock as illustrated in Figure 6.7 (c). The system now has a fixed point, and therefore stable operation is possible without an active phase control of the machines. The real part of the order parameter converges now to a fixed positive value as shown in the upper panel. Consequently, the average phase velocity $v_{\infty}$ goes to zero. Both the average phase velocity $v_{\infty}$ and the order parameter $r_{\infty}$ are plotted as a function of the coupling strength $K$ in Figure 6.7(d), demonstrating the onset of phase-locking above a critical coupling strength $K \geq K_{c} \approx 13 P_{0}$.

The synchronization transition is quantitatively analyzed in Figure 6.8 for the decentralization process introduced in section 6.1.2. We find that self-organized phase-locking is possible for lower transmission line capacities if additional but smaller and decentralized sources are present in the grid. This result is in accordance with the results shown in the previous section for model networks of regular, small-world and random topologies.

Figure 6.8(a,b,c,d) illustrates how the capability of the power grid to phase-lock is affected by the decentralization process for different values of $c$. Intuitively, the transmission lines connecting the power plants to the rest of the grid are heavily loaded and thus most likely to fail. Therefore stability would be increased just because these lines become less loaded 
if large generators are replaced by several smaller ones. This notwithstanding, decentralization itself, by its more distributed nature, supports phase-locking. To demonstrate this, we increase the transmission capacities of transmission lines that connect power plants to the grid by a factor $c$ compared to the capacities of the other transmission lines. Selforganized phase-locking is still promoted by decentralization for $c=2$ as demonstrated in Figure 6.8 $(\mathrm{a}, \mathrm{b})$. It is even promoted for $c=10$, completely compensating the ten-fold difference in the power output of large and small decentralized generators as demonstrated in Figure 6.8 $(\mathrm{c}, \mathrm{d}$,$) . Most interestingly the phase order parameter r_{\infty}$ increases with decentralization. At the same time, the average phase velocity $v_{\infty}$ decreases. The critical coupling strength $K_{c}$ for the onset of phase-locking thus decreases, i.e., phase-locking can be already realized with less transmission capacity as illustrated in Figure 6.8(e).

This is remarkable, as it has been questioned whether a network of many small, distributed power sources can be effectively synchronized without the help of a reference signal generated by large power grids (see, e.g., [70]). We conclude that at least for stationary operation, further decentralizing a grid promotes self-organized phase-locking.

\subsection{Synchronization time}

A sufficiently large coupling of the nodes leads to the phase-locking of all nodes of a power grid as demonstrated in the preceding sections. In this section we analyze the synchronization time, i.e., the time the grid needs to reach a stable phase-locked state for different degrees of decentralization. In real power grids this process has to be reasonably quick. Starting from an arbitrary state in the basin of attraction, the network relaxes to the stable phase-locked state with a characteristic time scale $\tau_{\text {sync }}$. For instance, Figure 6.9(a) illustrates the damped oscillations of the phase $\theta_{j}(t)$ of a power plant and a consumer in a quasi-regular grid with $K=10$ and $N_{P}=10$. In order to quantify the relaxation, we calculate the distance to the steady state

$$
d(t)=\left(\sum_{j=1}^{N} d_{1}^{2}\left(\theta_{j}(t), \theta_{j, \mathrm{st}}\right)+d_{2}^{2}\left(\dot{\theta}_{j}(t), \dot{\theta}_{j, \mathrm{st}}\right)\right)^{\frac{1}{2}},
$$

where the subscript 'st' denotes the steady state values. For the phase velocities $d_{2}$ denotes the common Euclidean distance

$$
d_{2}^{2}(\dot{\alpha}, \dot{\beta})=|\dot{\alpha}-\dot{\beta}|^{2}
$$

while the circular distance of the phases is defined as

$$
d_{1}(\alpha, \beta)=1-\cos (\alpha-\beta)
$$




\section{Decentralized power generation in future power grids}
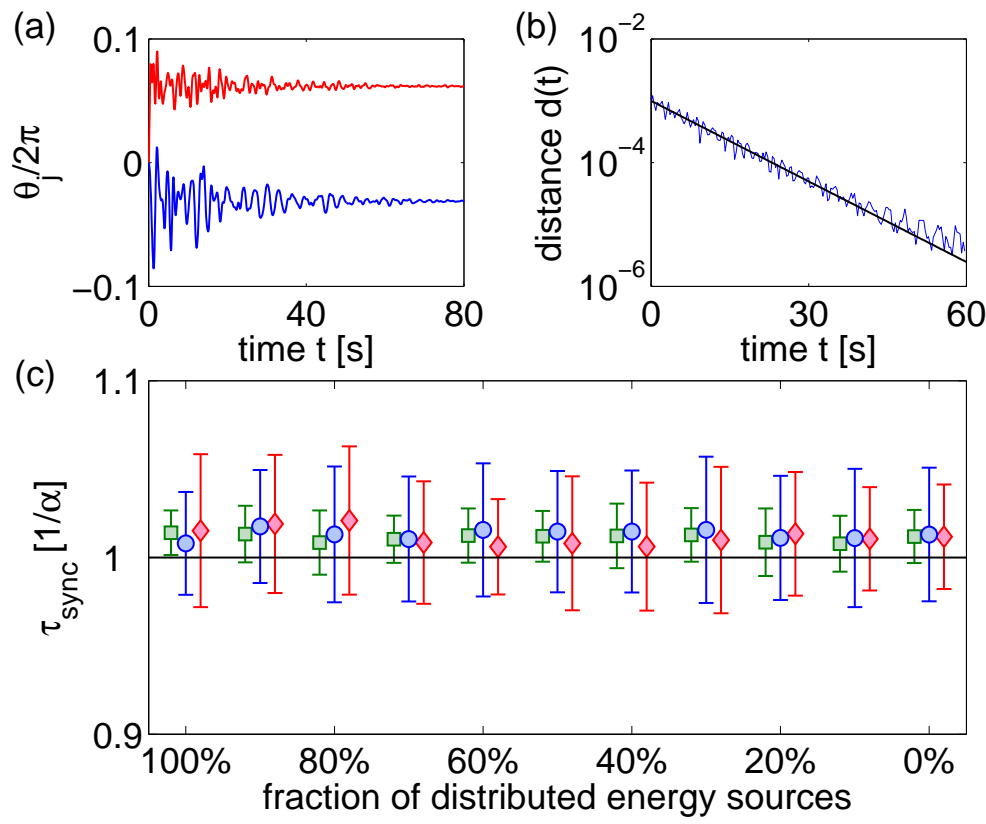

Figure 6.9: Relaxation to the phase-locked steady state. (a) Illustration of the relaxation process $\left(K / P_{0}=10\right.$ and $\left.N_{p}=10\right)$. We have plotted the dynamics of the phases $\theta_{j} / 2 \pi$ only for one generator (red) and one consumer (blue) for the sake of clarity. (b) Exponential decrease of the distance (cf. 6.13) to the steady state (blue line) and a fit according to $d(t) \sim e^{-t / \tau_{\text {sync }}}$ (black line). (c) The synchronization time $\tau_{\text {sync }}$ as a function of the fraction of decentralized energy sources $1-N_{P} / 10$ for a regular (०), a random $(\square)$ and a small-world grid $(\diamond)$. Cases where the system does not relax are discarded.

The distance $d(t)$ decreases exponentially during the relaxation to the steady state as illustrated in Figure 6.9(b). The black line is a fit with the function $d(t)=d_{0} \exp \left(-t / \tau_{\text {sync }}\right)$. Thus synchronization time $\tau_{\text {sync }}$ measures the local stability of the stable fixed point, being the inverse of the stability exponent $\lambda$ (cf. the discussion in section 5.2).

The dependance of the synchronization time on the structure of the network and the mixture of power generators is illustrated in Figure 6.9 (c). For several paradigmatic systems of Kuramoto oscillators it has been demonstrated that the time scale of the relaxation process depends crucially on the network structure $71-73$. Here, however, we have a network of damped second order oscillators. Therefore the relaxation is almost exclusively given by the inverse damping constant $\alpha^{-1}$. Indeed, we find

$$
\tau_{\text {sync }} \gtrsim \alpha^{-1} \text {. }
$$

For the given parameter values we observe neither a systematic dependence of the synchronization time $\tau_{\text {sync }}$ on the network topology nor on the number of large $\left(N_{P}\right)$ and small $\left(N_{R}\right)$ power generators. The mean value of $\tau_{\text {sync }}$ is always slightly larger than the relaxation constant $\alpha^{-1}$. Furthermore, also the standard deviation of $\tau_{\text {sync }}$ for different realizations of 
the random networks is at most $3 \%$ of the mean value.

\subsection{Robustness against dynamic perturbations}

Renewable energy sources possess a fluctuating energy output [4, 10, 11, 74]. In addition, also the local power consumption fluctuates depending on the decisions of the consumers. Therefore, power grids which include these power sources have to be especially robust against power fluctuations. Here we test the robustness of the four different topology classes (cf. section 6.1) against perturbations of the power demand of the consumers. With respect to the oscillator model (see [29]), perturbations on the consumers' side are equivalent to perturbations of the generators, such that we can restrict our analysis to adding a perturbation on the consumers' side for a certain period of time. As we demonstrated in section 5.3 power grids operate in the coexistence regime, at least during periods of high loads. The aim is now to quantify the maximum tolerable perturbations that does not lead to power outage of the grid, i.e., does not drive the system out of the basin of attraction of the stable fixed point. Real-world power grids have a large tolerance against such perturbations, otherwise power outages would occur frequently.

\subsubsection{Robustness of random network ensembles against dynamic perturbations}

We test the stability of the three different network structures described in section 6.1.1 against perturbations on the consumers' side. We perturb the system after it has reached a stable state and measure if the system relaxes back to a steady state after the perturbation has been switched off again. The perturbation is realized by an increased power demand of each consumer during a short time interval of $\Delta t=10 \mathrm{~s}$ as illustrated in the upper panels of Figure 6.10. Therefore the power balance condition (see 44.33) is violated and the system cannot remain in its stable state. After the perturbation is switched off again, the system either does or does not relax back to a steady state, depending on the strength of the perturbation. Examples for the dynamics of weak and strong perturbations are illustrated in Figure 6.10 (a,b).

These simulations are repeated 100 times for every value of the perturbation strength for each of the network topologies. We count the fraction of networks which are unstable, i.e, do not relax back to a steady state. The results are summarized in Figure 6.11 for the different network topologies. The Figure illustrates the fraction of unstable grids as a function of the perturbation strength and the fraction of decentralized power generation which is given by $N_{R} / 100$ where $N_{R}$ is the number of small decentralized generators in the grid. For all topologies, the situation with highest robustness against power perturbations is found if the power is generated by large power plants as well as small power generators 


\section{Decentralized power generation in future power grids}
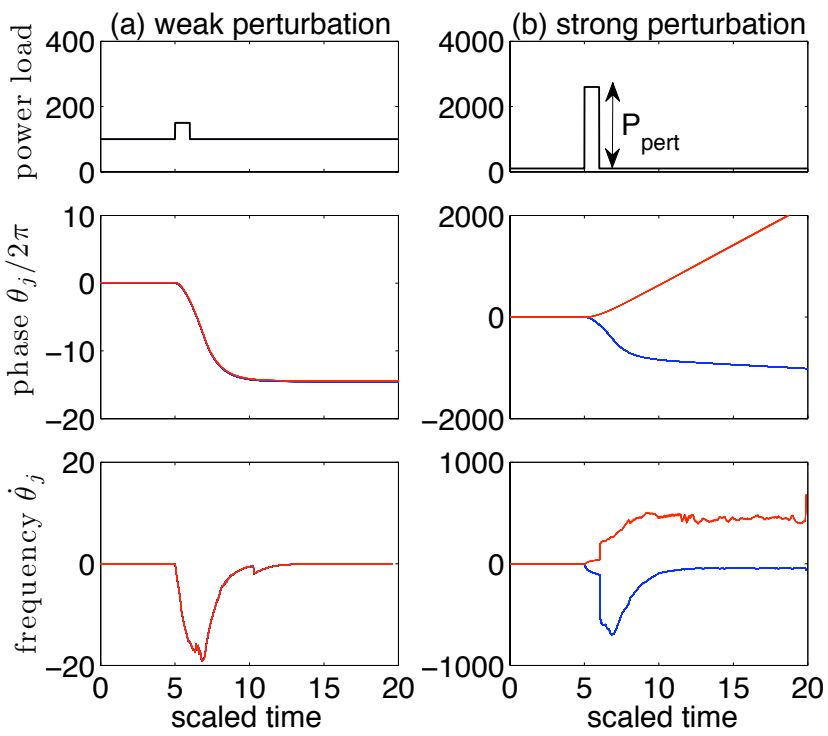

Figure 6.10: Effects of weak and strong perturbations. The upper panels show the timedependent power load of the consumers. A perturbation of strength $P_{\text {pert }}$ is applied in the time interval $t \in[5,6]$. The lower panels show the resulting dynamics of the phases $\theta_{j} / 2 \pi$ and the frequencies $\dot{\theta}_{j}$ of the consumers (blue lines) and the power plants (red lines). The dynamics relaxes back to a steady state after the perturbation for a weak perturbation (a), but not for a strong perturbation (b). In both cases we assume a regular grid with $N_{P}=10$.

with approximately $30 \%$ decentralization. A possible explanation is that the power output of large centralized sources is higher, which leads to a higher tolerance against power perturbations. On the other hand, a more distributed arrangement of power stations favors a stable phase-locked operation as demonstrated in section 6.2 .

Furthermore, the variability between the different realizations of the power grids is stronger for small values of $N_{P}$, i.e., few large power plants. The results do not change much for networks which many power sources (i.e. high $N_{P}$ ) because more power sources are distributed in the grid. Thus, the different realizations of the networks differ only weakly, and we can observe a sharp transition between tolerable and intolerable perturbations. This is different if only few large power plant are present in the network. For certain arrangements of power stations the system can reach a steady state even for strong perturbations. But the system can also fail to do so for only small perturbations if the power stations are clustered. This emphasizes the necessity for a careful planning of the topology of a power grid to guarantee maximum robustness. 


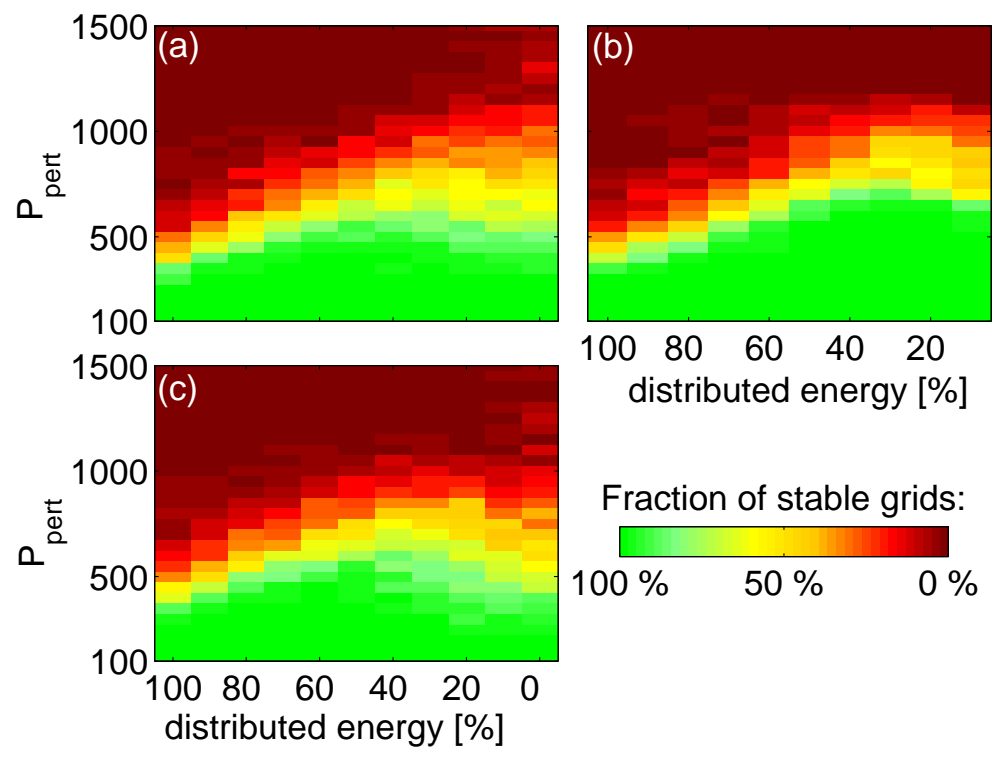

Figure 6.11: Robustness of a power grid. The panels show the fraction of random grids which are unstable against a perturbation as a function of the perturbation strength $P_{\text {pert }}$ and the fraction of decentralized energy $1-N_{P} / 10$. (a) Quasi-regular grid, (b) random network and (c) small-world network.

\subsubsection{Robustness of the British power grid against dynamic perturbations}

It was demonstrated in the previous subsection that the best situation in terms of a tolerable perturbation strength is found for about $30 \%$ distributed sources for all considered model networks. Here we test the stability against power perturbations for the topology of the British power grid. As in the previous section, we increase the demand of all consumers for a short period of time (here: $0 \mathrm{~s}-5 \mathrm{~s}$ ) as illustrated in Figure 6.12(a,b) and test whether the grid relaxes back to a steady state. We evaluate the robustness as a function of the perturbation strength $P_{\text {pert }}$ and the decentralization of the grid (cf. section 6.1.2) for two different values of $c$.

The results are summarized in Figure 6.12(c) for $c=2$ and in Figure 6.12(d) for $c=10$. We find that the maximally allowed perturbation strength shrinks with decentralization, but both grids are stable up to a perturbation strength a few times larger than the unperturbed load. A notable difference to the results in the previous section is that here both grids have no peak for the maximum tolerable perturbation. Instead the maximum tolerable perturbation always increases with less distributed power production. Furthermore, the British power grid has no difference of the sharpness of the transition between tolerable and intolerable perturbations. We assume that the differences in the response to such perturbations are due to the different decentralization processes. 


\section{Decentralized power generation in future power grids}
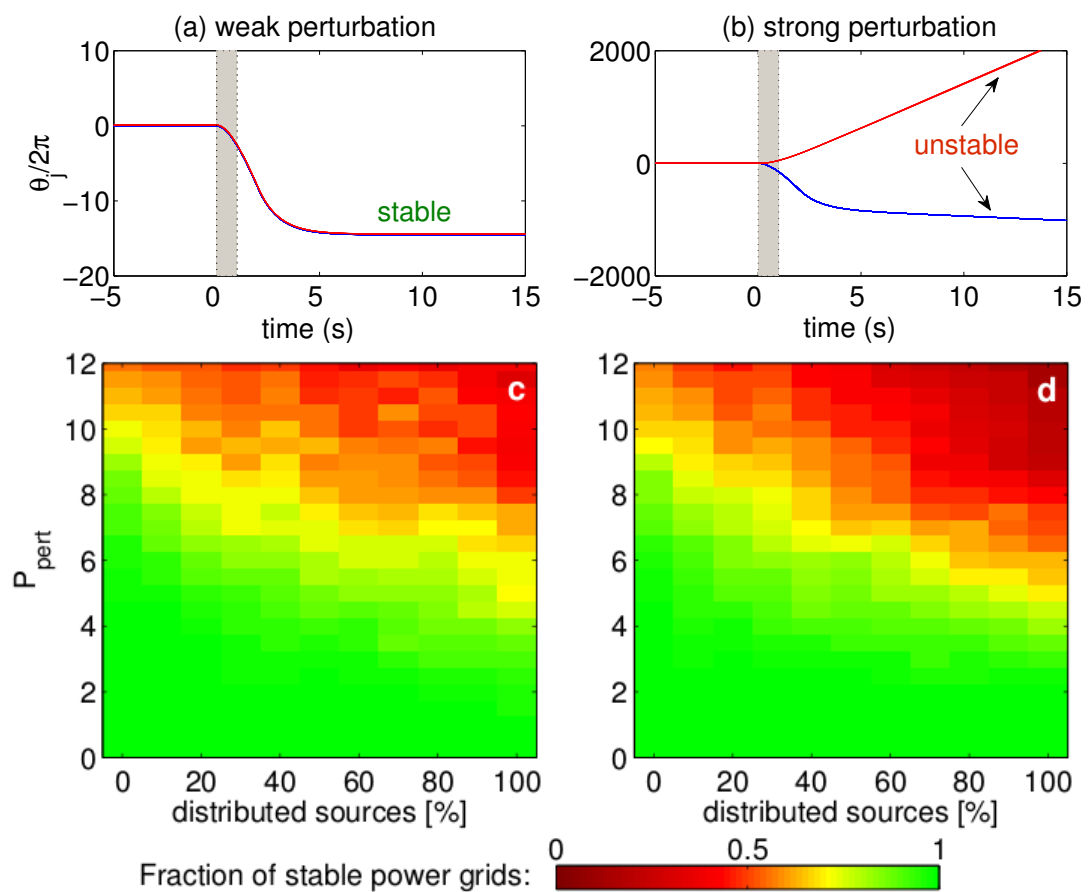

Figure 6.12: Robustness of the British power grid against perturbations in power demand. $(a, b)$ Time evolution of the phases of the generators (red) and consumers (blue) for weak and strong power perturbations in the time interval $0 \mathrm{~s}-5 \mathrm{~s}$ (shaded area). (c,d) The colormaps indicate the fraction of random grids which are unstable against a perturbation as a function of the perturbation strength $P_{\text {pert }}$ and the fraction of small distributed generators. Numerical results have been averaged over 100 realizations, where the replacing smaller power sources were randomly placed in the grid. Parameters are $K=20$ and $c=2$ in (c) and $K=20$ and $c=10$ in (d).

\subsection{Stability against structural damages}

Decentralization requires the addition of new transmission lines into the power grid 18 , 75]. This raises the still open question if the addition of new transmission lines makes the grid more stable, 61, 63, or not, 64. Here we analyze the stability of the British power grid against structural damages to its transmission lines. We have simulated the impact of the breakdown of single transmission lines on the capability of the power grid to maintain stable operation. An example is illustrated in Figure 6.13(a) for the British power grid, comparing the stability properties of a fully centralized grid with one where $10 \%$ of the power sources are decentralized. The importance of each link for the stability of the phase-locked state is indicated by the color of the lines. Green indicates non-crucial lines that can be removed without losing phase-locking for both grids. Black colors indicates bridges, whose removal disconnects the grids. Two transmission lines (blue) are crucial for the stability of the initial grid, but not anymore when the marked power plant is replaced by distributed generators. Red lines are crucial in both grids. 

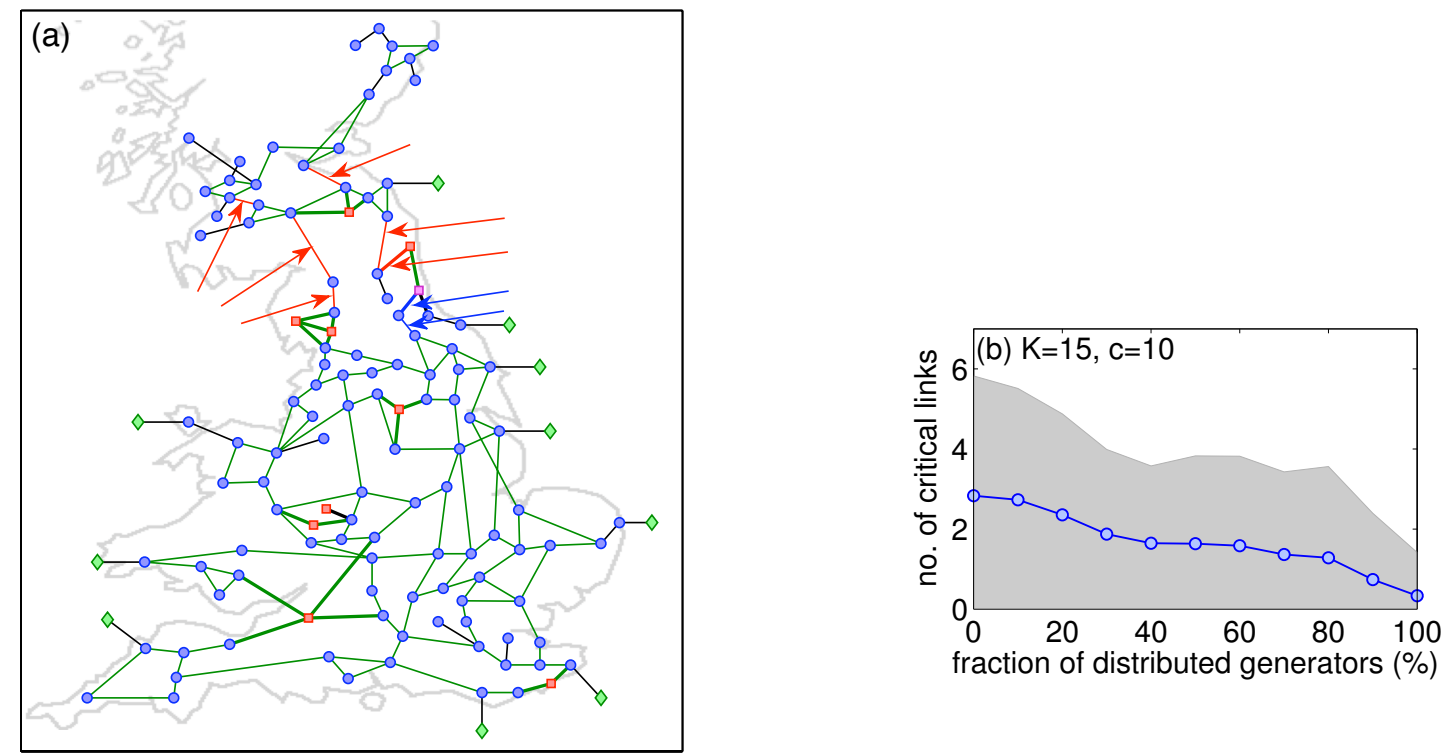

Figure 6.13: Structural stability of the British power grid. (a) The color of the links illustrates their relevance for the global structural stability of the power grid (see main text). Removing certain single links causes power outage: two links (blue arrows) are crucial for the stability of the initial grid, but not anymore when the marked power plant is replaced by distributed generators. Six links (red arrows) are crucial in both cases. Parameters are $K=12$ and $c=10$. To simulate the decentralization of the grid, we repeatedly replace one large centralized power plant by 10 small distributed generators as described in section 6.1.2. (b) Change of structural stability. The panel shows the number of critical links in the network, whose removal leads to a loss of synchrony and thus a major power outage, discarding bridges. Here, the coupling strength is fixed to $K=15$ and $c=10$. Quantities are averaged over 100 realizations, as in Fig. 6.12. The shaded area shows the standard deviation.

Extensive numerical simulations demonstrate how the decentralization of the British power grid affects the structural robustness of the grid. The results are illustrated in Figure 6.13(b). We find that the average number of critical links decreases significantly compared to systems with many small sources. Thus, decentralization leads to a higher stability against structural damages. Further inspection of the detailed consequences of removing links suggests a rough intuitive explanation: the probability of a global failure is highest when there is no pathway in the immediate neighborhood which can take over the respective power load. This is more often the case for grids with few, large power plants, as there are less transmission paths in the network. This issue will be analyzed in detail in the following chapter. We conclude that replacing large power plants by distributed generators may not only promote phase-locking but also increase the robustness of the power grid with respect to structural damages. 


\section{Decentralized power generation in future power grids}

\subsection{Summary and discussion}

In summary, we have analyzed how the ongoing decentralization of power grids due to the development of renewable energy sources effects the onset of phase-locking and the stability of power grids with complex topologies. We showed that the onset of phase-locking is promoted for lower line transmission capacities for decentralized power grids. We demonstrated this effect for all four considered grid topologies. Furthermore, the synchronization time, i.e., the time the power grid needs to reach the stable fixed point, is not negatively effected by decentralization. The synchronization time depends always crucially on the damping for all degrees of decentralization. We analyzed the robustness of the power grid against large-scale perturbations for all four considered grid topologies. We demonstrated that all grid topologies possess a high tolerance against power perturbations. However, the three general topologies (random, small-world and quasi-regular) possess the largest tolerance against power perturbations for approximately $30 \%$ of decentralization. The British power grid has a large, but with decentralization constantly decreasing tolerance against power perturbations. We assume that these different behaviors are due to the different decentralization process for the three general grid topologies and the British grid. We analyzed the structural stability of power grids. The structural stability is a global measure of the amount of critical transmission lines, i.e., transmission lines that would cause power outages if they fail. We counted the number of critical transmission lines that are indispensable for the operation of the grid. We showed that the number of critical transmission lines decreases with decentralization.

Intriguingly, we thus found two counteracting tendencies due to decentralization: As might be expected, networks become generally less stable against short-term, large-amplitude dynamic perturbations with increasing decentralization. At the same time, networks with more distributed power sources are less vulnerable to transmission line failures, i.e., structural damages. Taken together, our results indicate that decentralizing power sources may moderately decrease the grids' dynamic stability, but support the onset of self-organized phase-locking and the stability against structural damages. 


\section{Power outages and critical transmission lines}

Modern power grids consist of thousands of transmission lines [32]. It is likely that failures of transmission lines occur from time to time due to their vast number. Such an event typically has no impact on the normal operation of power grids. However, in periods of high loads the breakdown of single infrastructures such as transmission lines can cause a global cascade of failures implying large-scale outages with potentially catastrophic consequences [34 37, 76 84. An example of such a cascade of failures is the power outage in Western Europe triggered by the shutdown of one single transmission line in Germany in a period of high load in November 2006 [18]. In fact, most power outages can be traced back to failures of certain single transmission lines [85]. Periods of extreme loads are expected to become more likely in future grids as power from renewable sources, such as wind turbines, is often generated far away from the consumers (e.g. off-shore) [13 and moreover strongly fluctuating [2, 10, 11]. Maintaining a stable operation of the grid and minimizing the risk of power outages is thus an important challenge for the future operation of the grid.

In the previous chapter we analyzed the structural stability of power grids. We showed that that the number of critical transmission lines decreases with decentralization (cf. section 6.5). However, the structural stability does not provide any information why certain transmission lines are critical while others are not. In this chapter we identify which properties of transmission lines are crucial for their criticality. We analyze different topological and dynamic measures of transmission lines that may determine their importance for grid operation. We demonstrate that the edge redundancy is the key indicator for dynamic network robustness and that the relative remaining capacity prior to a line failure indicates outages with high accuracy. It is of fundamental importance to identify critical infrastructures that are indispensable for network operation [86]. Our findings indicate that potential outages resulting from the failure of specific lines may be predicted by topological and other stationary properties of normal network operation, i.e., the known state prior to any breakdown.

We illustrate the problem in the first section and introduce the simulation methods in the second. We distinguish between breakdowns in periods of low and high loads in the next two sections. For periods of low loads we show that the maximum flow after a breakdown can be predicted by simply rerouting the flow over the remaining paths of the network. This directly leads to the definition of quantitative measures of redundancy for single transmission lines. For periods of high loads we demonstrate that the redundant capacity 


\section{Power outages and critical transmission lines}
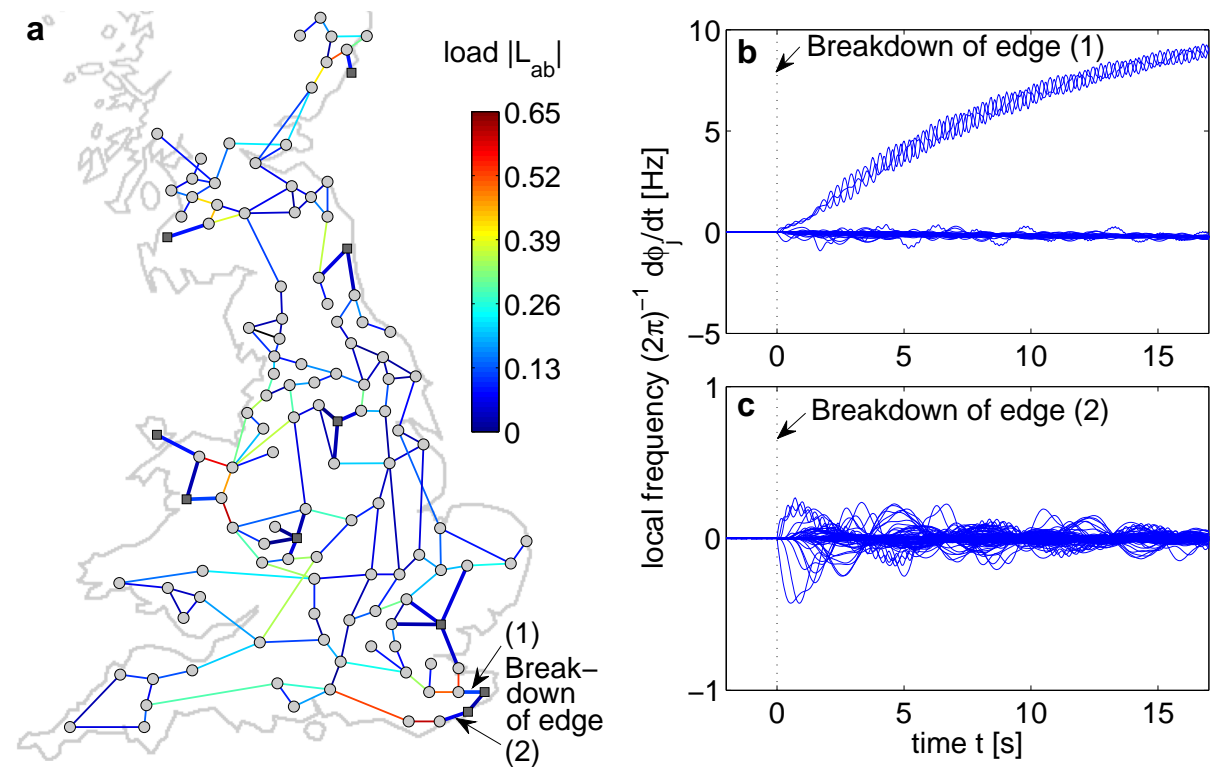

Figure 7.1: Breakdowns of neighboring transmission lines with similar loads may or may not cause large scale network outage. (a) Transmission line loads (color code) in the steady state in a coarse-grained model of the British high-voltage transmission grid (see Fig. 6.7 with $K_{0}=12 P_{0}$ ). Generators with $P=11 P_{0}$ are marked by a square, consumers with $P=-P_{0}$ are marked by a circle. $(\mathrm{b}, \mathrm{c})$ Dynamics after the breakdown of a single edge at $t=0$, the edges being marked by arrows in panel (a) with power outage in (b) and maintaining stable operation in (c). Is it possible to predict a large scale outage from simple characteristics before line breakdown?

is indeed a reliable predictor for the criticality of transmission lines. The chapter ends with a brief summary and discussion.

\subsection{Transmission line failures}

The addressed problem in this chapter is illustrated in Figure 7.1 for the oscillator power grid model introduced in section 4.2.4. The failure of two neighboring transmission lines with similar loads shown in Figure 7.1(a) may have completely different consequences for global network operation. Whereas the failure of one transmission line is causing a desynchronization of parts of the grid and consequently a large scale power outage as illustrated in Figure 7.1(b), the removal of the other transmission line leaves the entire network fully functional as illustrated in Figure 7.1(c). We find that such phenomena emerge across networks and line pairs even if the transmission lines (i) have the same capacity, are (ii) similarly located, e.g. in the same chain of transmission lines connecting two remote network nodes, and (iii) although only one edge apart from each other, as in the example. Such surprising examples yield the question of how to predict the impact of a local failure on the emergence of power outages on the basis of the topology and the 
prior state of the network?

\subsection{Simulation methods}

To reveal essential aspects of what determines the dynamic stability of complex supply networks, we consider the coarse-scale oscillatory model of power grids introduced in section 4.2.4. As a brief recapitulation, the equations of motion for the phase difference of each machine $i$ to the reference phase $\Omega t$ is given by

$$
\frac{d^{2} \theta_{i}}{d t^{2}}=P_{i}-\alpha \frac{d \theta_{i}}{d t}-\sum_{j} K_{i j} \sin \left(\theta_{j}-\theta_{i}\right) .
$$

In this model the power flow between two nodes of the network is given by

$$
F_{i j}=K_{i j} \sin \left(\theta_{j}-\theta_{i}\right)
$$

We performed direct numerical simulations of the model defined via the set of equations (7.1). Each simulation starts from a stationary phase-locked state of the intact network. We then simulate the dynamics after the breakdown of a single transmission line and test whether the network relaxes back to a stable operation. Specifically, we considered the British high-voltage transmission grid consisting of 120 nodes and 165 transmission lines [19, 37]. For each out of 100 random realizations, we fixed the network topology by randomly selecting ten nodes to be generators with power production of $P_{i}=+11 P_{0}$. All other nodes are consumers with power consumption of $P_{i}=-P_{0}$ with $P_{0}=1 \mathrm{~s}^{-2}$ (cf. section 6.1.2). Edges connecting generator nodes to the remaining grid are assumed to have a larger transmission capacity $K_{i j}=10 K_{0}$, marked by thick lines in Figure 7.1(a) than the remaining edges with $K_{i j}=K_{0}$ to compensate for otherwise trivially occurring overloads. For the statistical analysis (compare Figure 7.4 and Table 7.1) we thus generated 100 network realizations with various generator positions, thus testing 16.500 edges in total. We note that the network allows for a stable operation only if the transmission capacity exceeds a certain threshold value, $K_{0} \geq K_{c}$ (cf. section 6.2) 19, 58]. Networks not supporting a steady state before any edge breakdown were not considered.

\subsection{Breakdowns of transmission lines in periods of low loads}

To understand the effects of damages, we first consider power grids with $F_{i j} \ll K=$ $30 \mathrm{rms}^{-2}$, such that the transmission lines are far away from being overloaded, i.e., the system is far away from being in the globally unstable regime (cf. section 5.3). Here, the breakdown or removal of a single non-bridge transmission line leads to a redistribution of 


\section{Power outages and critical transmission lines}
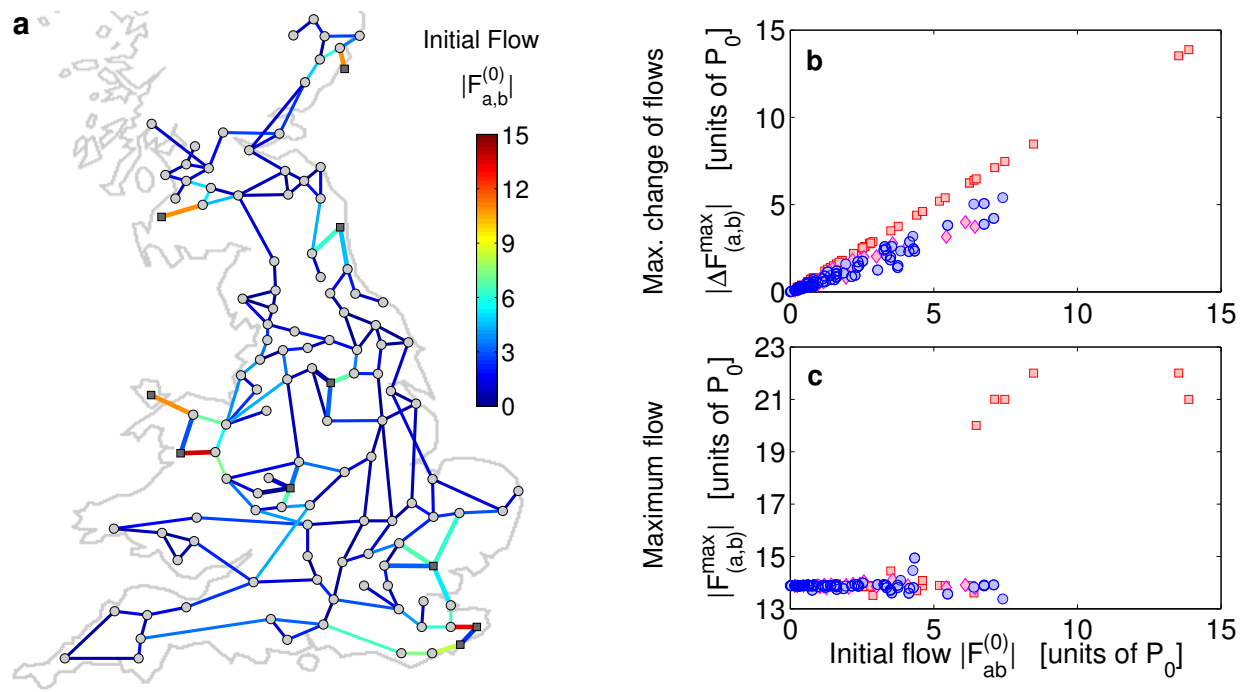

Figure 7.2: Change of network flows after the removal of one transmission line during normal operation. (a) Initial network flows $\left|F_{a, b}^{(0)}\right|$ in the unperturbed steady state in a coarse-grained model of the British high-voltage transmission grid. (b,c) Change of the network flows when one of the non-bridge edges $(a, b)$ is removed. Plotted is (b) the maximum change (cf. equation 7.3) and (c) the maximum value (cf. equation 7.4) of the network flows as a function of the load of the removed edge. We classify the removed edge according to their local edge connectivity $\lambda(a, b)$ as illustrated in Fig. 7.3 The maximum flow change $\left|\Delta F_{(a, b)}^{\max }\right|$ shown in (b) exactly equals the flow of the removed edge $\left|F_{a, b}^{0}\right|$ when there is only one redundant path, i.e. $\lambda(a, b)=2$, with parallel flow (red squares). The maximum network flow $\left|F_{(a, b)}^{\max }\right|$ after the removal shown in (c) is large when $\left|F_{a b}^{(0)}\right|$ is large and the local edge connectivity is small, i.e. $\lambda(a, b)=2$ (red squares and magenta diamonds). The load $F_{a, b}$ is given in units of the power $P_{0}$. The transmission capacity is $K_{0}=30 \mathrm{~s}^{-2}$. All other parameter values and number of generators and consumers are the same as in Fig. 7.1 .

the network flows, but does not induce a dynamical instability. We analyze how the change of network flows depend on the characteristics of the removed edge.

The flows in a coarse-grained model of the British power grid in a stable operation are illustrated in Figure 7.2(a). Each transmission line is far from being overloaded for the given parameters. We then remove one non-bridge edge $(a, b)$ and analyze how the flow over the remaining edges $(i, j)$ changes. In particular, we consider the maximum change of the flows

$$
\Delta F_{(a, b)}^{\max }:=\max _{i j}\left(F_{i j}^{(1)}-F_{i j}^{(0)}\right)
$$

and the maximum absolute value of the flow in the modified network

$$
F_{(a, b)}^{\max }:=\max _{i j}\left(F_{i j}^{(1)}\right) .
$$



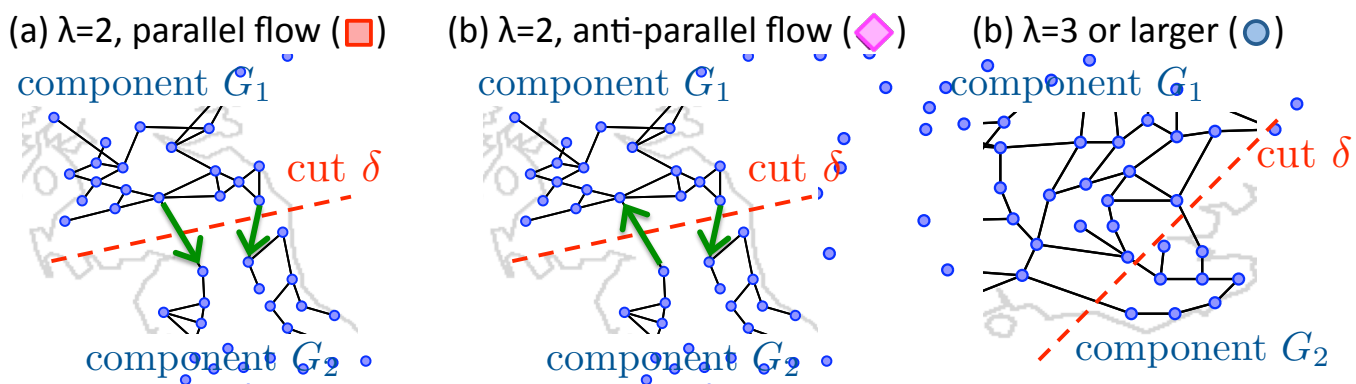

Figure 7.3: Network motifs and the definition of local edge connectivity $\lambda(a, b)$ between two nodes $a$ and $b$. (a,b) Cutting two links (green) disconnects the network. Nodes in the two different components $G_{1}$ and $G_{2}$ thus have a local edge connectivity of at most two, $\lambda \leq 2$. Furthermore, it is of fundamental importance for network robustness, whether the flow over the two links is parallel or anti-parallel as indicated by the direction of the arrows. (c) Cut between two components with a higher local edge connectivity, here $\lambda=3$.

Here, the superscripts (0) and (1) denote the flows before and after the removal, respectively.

When an edge $(a, b)$ is removed, the load $F_{a, b}^{(0)}$ has to be rerouted. Thus the change of loads in the other edges $(i, j)$ depends on two quantities. First, the original load of the edge $F_{a, b}^{(0)}$ itself is decisive as this is the load that has to be rerouted. As we are never rerouting more than the initial load of the removed edge $(a, b)$, we find the inequality

$$
\left|\Delta F_{(a, b)}^{\max }\right| \leqslant\left|F_{(a, b)}^{(0)}\right| \text {. }
$$

The second decisive quantity is the redundancy of the removed edge. We introduce the local edge connectivity $\lambda(a, b)$ as a topological measure for edges. The local edge connectivity of an edge $(a, b)$ that connects the nodes $a$ and $b$ is defined as the minimum number of edges, that would need to be cut to completely disconnect the nodes $a$ and $b$ [87], such that they are in two different components of the network as illustrated in Figure 7.3 . If we have $\lambda(a, b)=2$ we can further distinguish two scenarios. The power flow in the two edges can be parallel or antiparallel. The scenario of parallel flow is illustrated in Figure 7.3(a), for antiparallel flow in Figure 7.3(b). A third scenario with $\lambda(a, b)=3$ is shown in Figure 7.3 (c).

If the local edge connectivity is low, i.e. $\lambda(a, b)=2$, the complete load $F_{(a, b)}^{(0)}$ has to be rerouted over one edge $(c, d)$, that belongs to the alternative shortest path from $a$ to $b$. Then

$$
\left|F_{(c, d)}^{(1)}\right|-\left|F_{(c, d)}^{(0)}\right|= \pm\left|F_{(a, b)}^{(0)}\right|
$$

depending on whether the flow on $c \rightarrow d$ is parallel or anti-parallel to the flow $a \rightarrow b$. If $\lambda(a, b) \geqslant 3$ there are several alternative pathways along which $F_{(a, b)}^{(0)}$ can be rerouted. 


\section{Power outages and critical transmission lines}

The quantities $\left|\Delta F_{(a, b)}^{\max }\right|$ and $\left|F_{(a, b)}^{\max }\right|$ are plotted in Figure $7.2(\mathrm{~b}, \mathrm{c})$ as a function of the load of removed edge $\left|F_{a b}^{(0)}\right|$. The colored symbols refer to the different scenarios depicted in Figure 7.3. Red squares symbolize edges with $\lambda(a, b)=2$ and parallel flow, magenta diamonds edges with $\lambda(a, b)=2$ and antiparallel flow and blue circles edges with $\lambda(a, b) \geq$ 3. We thus find that all red squares are on the diagonal for the maximum change of flows as expected. Results are shown for one realization of the network.

More important for network stability is the maximum absolute load defined in equation 7.4 . It is plotted in Figure 7.2 (c). For most removed edges $(a, b)$ this quantity is only weakly affected because the flow $F_{(a, b)}^{(0)}$ has to be rerouted through a path that has a lot of free capacity. A large increase of the maximum flow is only found if the removed edge was heavily loaded and weakly redundant, i.e. $\lambda(a, b)=2$. Edges satisfying these two conditions have a strong impact on the flows in a steady state of the network. However, this does not imply that they are equally important for the existence of a steady state.

\subsection{Breakdowns of transmission lines in periods of high loads}

In the previous section we analyzed scenarios of low loads. Such a situation is a priori not very dangerous, because the transmission lines have a lot of free unused transmission capacity. In case of breakdowns there are thus many possibilities to redirect the power flow. We showed that only scenarios with only one alternative pathway are potentially dangerous.

In this section we analyze scenarios where the power grid is heavily loaded. In such a scenario the power grid is vulnerable to large scale outages triggered by breakdowns of single transmission lines. It is also expected that scenarios with heavy load will occur more frequently in power grids with lots of sustainable power sources [88, 89].

\subsubsection{Stability or large scale outage?}

It is well established, that in period of high loads, the breakdown of a single edge can trigger a large scale outage in power grids, with potentially catastrophic effects $[37,76$, 79, 82. However, to find the criteria which determine whether an edge is critical for the operation of the grid or not is still an open challenge. To analyze how to identify these edges we simulated the dynamics of a large set of model grids after the breakdown of an edge $(a, b)$, sampling all edges in the network. In particular, we call an edge "stable" if the network relaxes back to an attractive stable phase-locked state, i.e. a state with $\dot{\theta}_{i}=0$ for all $i$. Otherwise we call the edge "critical". Examples for both a stable and an unstable scenario are shown in Figure 7.1(b,c). 

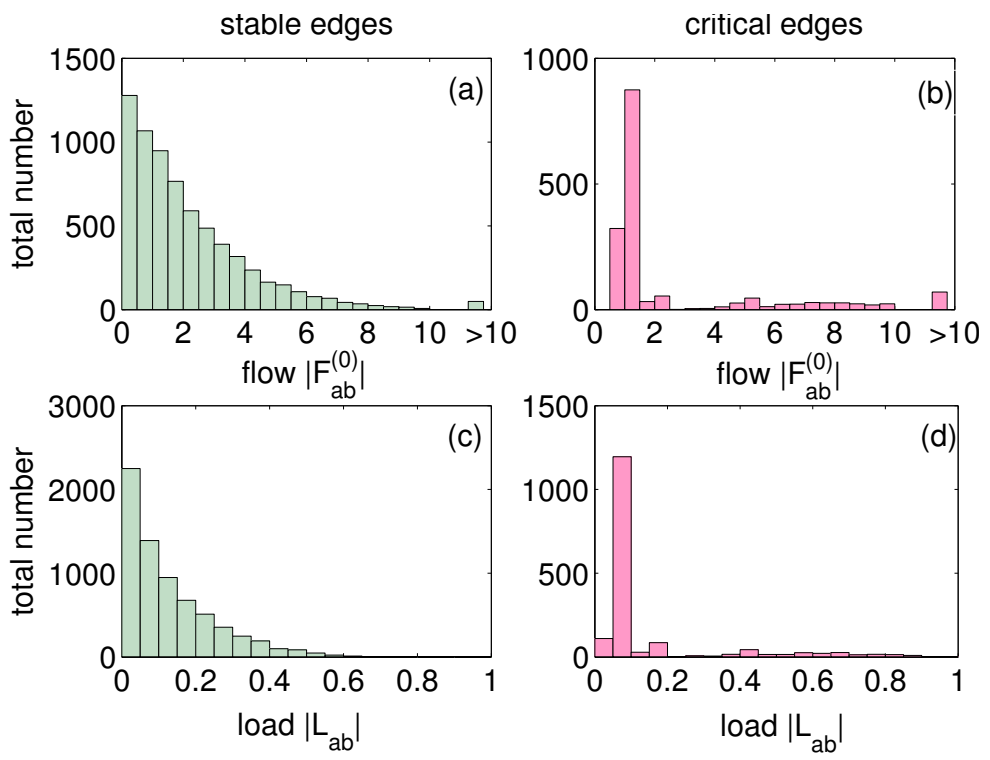

Figure 7.4: A statistical analysis demonstrates that critical edges cannot be reliably identified by the load or the power flow. Shown are histograms of the power flow $\left|F_{a b}^{(0)}\right|(\mathrm{a}, \mathrm{b})$ and the load $L_{a b}=F_{a b}^{(0)} / K_{a b}(\mathrm{c}, \mathrm{d})$ of stable (left hand side) and critical edges (right hand side) for edges with $\lambda(a, b)=2$. Results are collected for 100 random realizations of the British grid. The flow is given in units of $P_{0}$.

Intuitively, it seems likely that heavily loaded edges are most critical for grid operation. However, a systematic statistical analysis reveals that neither the power flow $F_{(a, b)}$ nor the load $L_{a b}=F_{a b} / K_{a b}$ is a reliable indicator to decide whether an edge $(a, b)$ is critical or not. This is illustrated in Figure 7.4. We find edges to be stable despite being heavily loaded (a,c) as well as critical edges which are only weakly loaded (b,d) for both the load illustrated in Figure 7.4(a,b) and the power flow illustrated in Figure 7.4(c,d). Moreover, among the critical edges we find weakly loaded ones even more often than heavily loaded ones. Thus, load and power flow are not useful measures in determining the stability of an edge.

\subsubsection{Redundancy in power grids}

Instead of the absolute flow or the relative load, redundancy is the key concept to detect critical edges. A general criterion of redundancy in technical systems is how many alternatives are available if a piece of infrastructure fails. In a supply network, this is quantified by the local edge connectivity $\lambda(a, b)$, which counts the number of independent paths between two nodes $a$ and $b$ [87]. If $\lambda(a, b)=1$ there is no redundant path such that a breakdown of the edge $(a, b)$ inevitably causes desynchronization. On the contrary, the breakdown of a highly redundant edge with $\lambda_{(a, b)} \geq 3$ causes a global desynchronization only in few 


\section{Power outages and critical transmission lines}
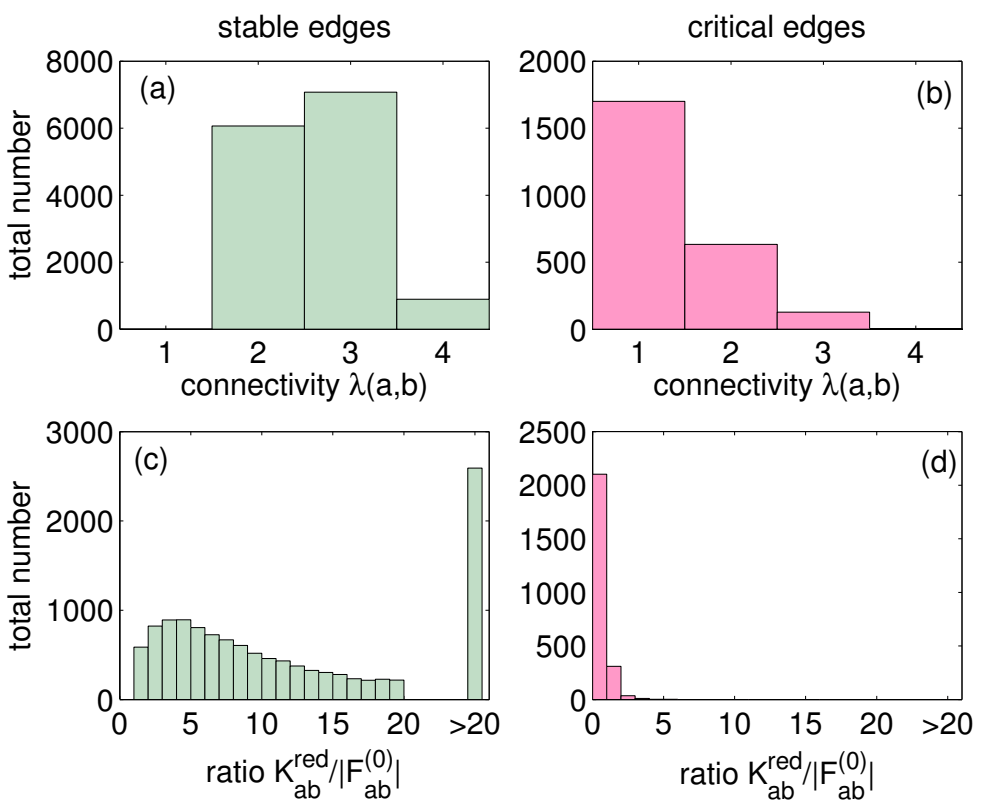

Figure 7.5: A statistical analysis shows that critical edges can be reliably identified by the local edge connectivity. Shown are histograms of the local edge connectivity $\lambda(a, b)(a, b)$ and the ratio of the redundant capacity $K_{(a, b)}^{\text {red }}$ and the flow $\left|F_{a b}\right|(\mathrm{c}, \mathrm{d})$ of stable (left hand side) and critical edges (right hand side). Critical edges can be reliably identified by low values of $K_{(a, b)}^{\mathrm{red}} /\left|F_{(a, b)}\right|$. Results are collected for 100 random realizations of the British grid.

exceptional cases as illustrated in Figure $7.5(\mathrm{a}, \mathrm{b})$. We thus conclude that the local edge connectivity yields good predictions for the criticality of edges, certainly for $\lambda(a, b)=1$ and $\lambda(a, b) \geq 3$. It is thus a more reliable predictor than the load or the power flow. However, for $\lambda(a, b)=2$ both stable and critical edges can still be found with a noteworthy probability.

Thus, a more careful treatment is required to obtain a reliable predictor for the stability of edges, which works also for the intermediate scenario $\lambda(a, b)=2$. If the edge $(a, b)$ fails, the flow $F_{a b}^{(0)}$ has to be rerouted over alternative paths in the network. However, the edges along these paths have only a limited residual capacity to take over this flow. Therefore we define the redundant capacity $K_{a b}^{\mathrm{red}}$ of an edge $a \rightarrow b$ as the maximum additional flow that the network can transmit from node $a$ to $b$. Here, the maximum is defined in a purely graph-theoretical way, i.e. we search for a flow which satisfies $\left|F_{i j}\right|<K_{i j}$ for all $i, j$ and flow conservation at every node. This definition depends only on the structure of the network and the steady state flow and can thus be applied to every model of a supply network. It does not take into account whether such a flow is dynamically possible or stable for the particular network type under consideration.

Furthermore, we note that the redundant capacity $K_{a b}^{\mathrm{red}}$ of an edge $(a, b)$ depends on the direction of the flow of this particular edge (see Figure 7.3 ). It is thus directed, i.e. $K_{a b}^{\mathrm{red}} \neq$ 


\begin{tabular}{|cc|rr|rr|}
\hline & Edge is & \multicolumn{2}{|c|}{ critical } & \multicolumn{2}{|c|}{ stable } \\
\hline$\lambda(a, b)=1$ & & 1700 & $(10.3 \%)$ & 0 & $(0.0 \%)$ \\
\hline$\lambda(a, b)=2$ & $K_{a b}^{\mathrm{red}}<h F_{a b}^{(0)}$ & 606 & $(3.7 \%)$ & 398 & $(2.4 \%)$ \\
\cline { 2 - 5 } & $K_{a b}^{\mathrm{red}} \geq h F_{a b}^{(0)}$ & 27 & $(0.2 \%)$ & 5669 & $(34.4 \%)$ \\
\cline { 1 - 5 }$\lambda(a, b) \geq 3$ & $K_{a b}^{\mathrm{red}}<h F_{a b}^{(0)}$ & 93 & $(0.6 \%)$ & 32 & $(0.2 \%)$ \\
\cline { 2 - 6 } & $K_{a b}^{\mathrm{red}} \geq h F_{a b}^{(0)}$ & 39 & $(0.2 \%)$ & 7936 & $(48.1 \%)$ \\
\hline
\end{tabular}

Table 7.1: Proposed simple classification of critical vs. stable edges. Out of the total of 16500 edges, $96.99 \%$ have been correctly classified for a threshold value of $h=1.8$. Parameters and the data set are the same as in Fig. 7.5 .

$K_{b a}^{\mathrm{red}}$. This is immediately clear from the fact that the flow is directed. If an edge $(i, j)$ is fully loaded in the sense that $F_{i j}=-F_{j i}=K_{i j}$, then it cannot transmit any additional flow from node $i$ to node $j$. However, it can transmit additional flow in the other direction from $j$ to $i$. In fact, this would lower the net flow over the edge $(i, j)$ and thus the load of the edge.

The redundant capacity of the edge $(a, b)$ is calculated using a modification of the EdmondsKarp algorithm [87]. As discussed above, $K_{a b}^{\mathrm{red}} \neq K_{b a}^{\mathrm{red}}$ such that the algorithm works with directed graphs. In the following we present the work steps of the algorithm.

Input: $\quad$ Capacity matrix $K$, Initial flow matrix $F$, edge $a \rightarrow b$

Output: Redundant capacity $K_{a b}^{\mathrm{red}}$ of the edge $a \rightarrow b$.

Step 1: Delete the edge $(a, b)$ from the effective network: $K_{a b}, K_{b a}, F_{a b}, F_{b a} \leftarrow 0$.

Step 2: $\quad$ Initialize $K_{a b}^{\mathrm{red}} \leftarrow 0$.

Step 3: $\quad$ Calculate the residual capacity matrix $K^{f} \leftarrow K-F$.

Step 4: Construct a shortest path $p$ from $a$ to $b$ in the directed graph defined by the capacity matrix $K^{f}$.

Step 5: If there is no path $p$ from $a$ to $b$ : STOP.

Step 6: Calculate the maximum available capacity along the path $p$ : $K_{\max }^{f} \leftarrow \max _{(i \rightarrow j) \in p} K_{i j}^{f}$.

Step 7: $\quad$ Increase the flow along the path $p: F_{i j} \leftarrow F_{i j}+K_{\max }^{f}$ for all edges $(i \rightarrow j) \in p$.

Step 8: $\quad$ GOTO step 3.

If edges have low redundant capacities they are with a higher probability critical than stable, if they have high redundant capacities they are almost always stable. A reliable criterion to identify critical edges is thus given by the ratio $K_{(a, b)}^{\mathrm{red}} /\left|F_{a b}\right|$ as illustrated in Figure 7.5 (c,d). In particular we propose the following classification system for critical and 
stable edges:

$$
\begin{aligned}
& K_{a b}^{\mathrm{red}}<h \times\left|F_{a b}^{(0)}\right| \Rightarrow \text { predicted to be critical, } \\
& K_{a b}^{\mathrm{red}} \geq h \times\left|F_{a b}^{(0)}\right| \Rightarrow \text { predicted to be stable, }
\end{aligned}
$$

where $h$ is a threshold value that can be optimized for the specific task.

We find for the British power grid that if the redundant capacity for an edge is higher than a threshold value of $h=1.8$ times the flow of an edge $\left(K_{(a, b)}^{\mathrm{red}} \geq 1.8 \times F_{(a, b)}^{(0)}\right)$, the edge is almost always stable. This holds for all values of the local edge connectivity. For redundant capacities below that threshold we find that edges have a higher probability to be critical than stable. Bridges with local edge connectivity $\lambda(a, b)=1$ are of course always critical. The results are summarized in Table 7.1 .

\subsubsection{Performance of the statistical testing methods}

In this section we analyze the performance of the redundant capacity as a predictor for the criticality of edges in detail and compare it with other possible predictors. We show that it is possible to identify or classify critical infrastructures by the ratio of the redundant capacity $K_{a b}^{\text {red }}$ and the flow $\left|F_{a b}^{(0)}\right|$. To evaluate the performance of such a classification scheme we must first define the possible outcomes of a prediction, where we distinguish between two systematically different kinds of errors:

True positive: $\quad$ Edge is predicted critical and is critical.

False positive: $\quad$ Edge is predicted critical but is stable.

False negative: Edge is predicted stable but is critical.

True negative: $\quad$ Edge is predicted stable and is stable.

Generally it is impossible to rule out both false negative and false positive predictions such that a compromise must be achieved. In the current setting, the number of false negative predictions can be minimized by choosing a high value of $h$, while the number of false positive predictions can be minimized by choosing a small value of $h$.

A common evaluation of the performance of a classifier is given by the so-called receiver operating characteristics (ROC) 90]. Here, one considers the true positive rate of the test, also called the sensitivity (SEN)

$$
\mathrm{SEN}:=\frac{\text { \#true positive predictions }}{\text { \#true positive predictions }+\# \text { false negative predictions }}
$$

and the false positive rate (FPR)

$$
\mathrm{FPR}:=\frac{\# \text { false positive predictions }}{\# \text { false positive predictions }+\# \text { true negative predictions }}
$$




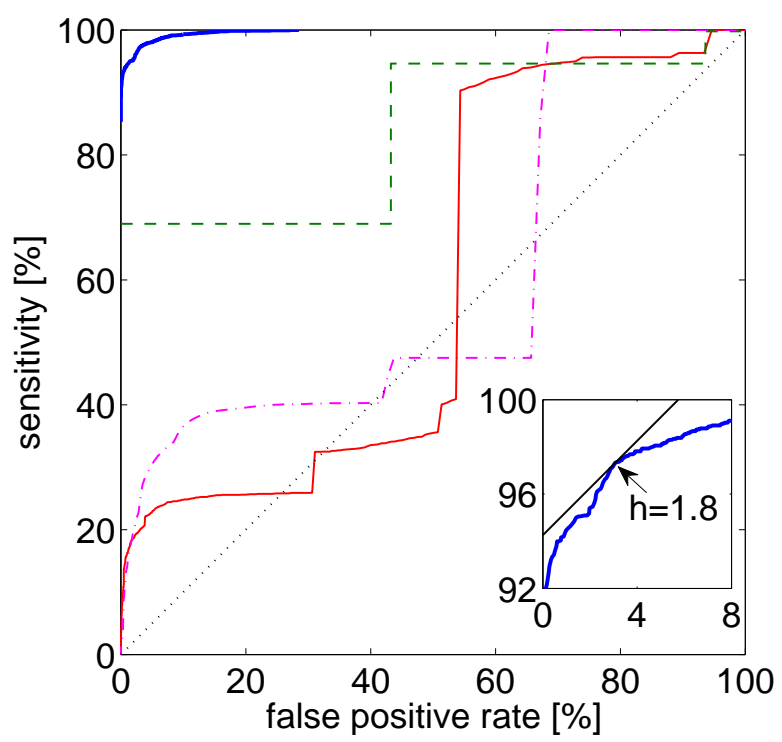

Figure 7.6: Receiver operating characteristics (ROC) of different classifier systems for critical and stable edges. The ROC curve shows the sensitivity (SEN) of the classifier vs. the false positive rate (FPR) for various values of the threshold value $h$. The proposed classification (cf. equation 7.7 based on the ratio $K_{a b}^{\mathrm{red}} /\left|F_{a b}\right|$ (thick blue line) closely approaches the perfect values $(0,1)$. Other possible classification schemes based on the edge connectivity $\lambda(a, b)(--)$, the load $L_{a b}(-)$ or the absolute flow $\left|F_{a b}\right|(-\cdot-)$ clearly perform worse. The inset shows a magnification around the point $(\mathrm{FPR}, \mathrm{SEN})=(0,1)$. The threshold $h$ is optimized by choosing the point where the ROC-curve has slope of 1 (marked by an arrow). The curves are based on the simulation of 100 random realizations of the British power grid with heterogeneous generation (cf. section 7.2).

For a perfect classifier, the ROC is a point at $(\mathrm{FPR}, \mathrm{SEN})=(0,1)$. If the classification depends on a threshold value $h$ one can plot SEN vs. FPR for different values of $h$ as in Figure 7.6. Here, $h$ can be optimized to the task of the test. For a fully random classification this ROC-curve would yield a straight line with slope 1 through the origin. Therefore, a classifier is judged to be better, the nearer the ROC-curve approaches the point $(0,1)$, i.e., the upper left corner of the plot. Alternatively, one can judge the classifier by the area under the ROC-curve [90].

We propose to identify critical infrastructures by the ratio of the redundant capacity $K_{a b}^{\text {red }}$ and the flow $F_{a b}^{(0)}$ (cf. (7.7)). The ROC shown in Figure 7.6 illustrates the performance of the load (red line), the power flow (magenta line), the local edge connectivity (green line) and the redundant capacity (blue line) as a predictor for different values of $h$. We find that for both the load and the power flow we either have very low false positive rates of under $20 \%$ combined with a low sensitivity around $20 \%$ or a high sensitivity over $80 \%$, but combined with a high false positive rate of over $50 \%$ depending on the value of $h$. None of these scenarios are reliable as a predictor. Thus there exists no value of $h$ for which either the load or the power flow is a reliable predictor. 


\section{Power outages and critical transmission lines}

\begin{tabular}{|c|c|c|c|}
\hline (a) Edge is & critical & stable & \\
\hline$K_{a b}^{\mathrm{red}}<h_{1} F_{a b}^{(0)}$ & 2399 & 430 & $\mathrm{PPV}=84.80 \%$ \\
\hline$K_{a b}^{\mathrm{red}} \geq h_{1} F_{a b}^{(0)}$ & 66 & 13605 & $\mathrm{NPV}=99.52 \%$ \\
\hline & $\mathrm{SEN}=97.32 \%$ & $\mathrm{SPE}=96.94 \%$ & \\
\hline (b) Edge is & critical & stable & \\
\hline$K_{a b}^{\mathrm{red}}<h_{2} F_{a b}^{(0)}$ & 2193 & 0 & $\mathrm{PPV}=100.0 \%$ \\
\hline$K_{a b}^{\mathrm{red}} \geq h_{2} F_{a b}^{(0)}$ & 272 & 14035 & $\mathrm{NPV}=98.10 \%$ \\
\hline & $\mathrm{SEN}=88.97 \%$ & $\mathrm{SPE}=100.0 \%$ & \\
\hline (c) Edge is & critical & stable & \\
\hline$K_{a b}^{\mathrm{red}}<h_{3} F_{a b}^{(0)}$ & 2465 & 3807 & $\mathrm{PPV}=39.30 \%$ \\
\hline$K_{a b}^{\mathrm{red}} \geq h_{3} F_{a b}^{(0)}$ & 0 & 10228 & $\mathrm{NPV}=100.0 \%$ \\
\hline & $\mathrm{SEN}=100.0 \%$ & $\mathrm{SPE}=72.87 \%$ & \\
\hline
\end{tabular}

Table 7.2: Classification of critical and stable edges according to the ratio $K_{a b}^{\mathrm{red}} /\left|F_{a b}^{(0)}\right|$ for three different values of the threshold $h$. (a) Threshold value $h_{1}=1.8$ for which the ROC curve has a slope of 1. (b) No false positive results occur for low threshold value $h_{2}=1.18$. (c) No false negative results occur for a high threshold value $h_{3}=5.76$. The specificity SPE is defined as SPE:=1-FPR. PPV and NPV denote the positive predictive value (cf. (7.10) ) and the negative predictive value (cf. (7.11)), respectively. Shown is the contingency table summarizing the results for 100 random realizations of the British power grid with heterogeneous generation (cf. section 7.2.

For the local edge connectivity we have either very low false positive rates down to $0 \%$ combined with a sensitivity of $68 \%$ or a high sensitivity over $90 \%$ combined with a false positive rate of over $40 \%$. The local edge connectivity is thus clearly a more reliable predictor than the load or the power flow. However, the redundant capacity performs even better as it comes closest to the desired point $(0,1)$. Figure 7.6 reveals that this classifier performs extremely well: It closely approaches the perfect operation point $(\mathrm{FPR}, \mathrm{SEN})=$ $(0,1)$.

Finally, the threshold value $h$ can be chosen according to the specific application of the classification system. A common choice is the point at which the slope of the ROC-curve is 1 [90]. This represents a compromise between the conflicting goals of large sensitivity and small false positive rate. For the present network data set this strategy is illustrated in the inset of Figure 7.6 , it yields the value $h=1.8$ for the redundant capacity. A different strategy would be to minimize false negative results such that every critical link is detected. For the given network data the number of false negative results can be reduced to zero for $h \geq 5.76$. This strategy reveals all critical links but also yields a comparably large number of false positive predictions. Similarly, the number of false positive results can be reduced to zero by choosing a low threshold value $h \leq 1.18$ at the expense of a comparably low sensitivity. The results are summarized in the contingency Table 7.2, In addition to the absolute number of true and false predictions, we also give some statistical quantities to 
characterize the classifier: the sensitivity ( cf. (7.8)), the specificity SPE $:=1-\mathrm{FPR}$ (cf. (7.9) ) and the positive predictive value (PPV)

$$
\mathrm{PPV}:=\frac{\# \text { true positive predictions }}{\# \text { true positive predictions }+\# \text { false positive predictions }}
$$

and the negative predictive value (NPV)

$$
\mathrm{NPV}:=\frac{\text { \#true negative predictions }}{\text { \#true negative predictions }+ \text { \#false negative predictions }}
$$

show that the flow and the redundant capacity correctly predict the stability of the network in $99.33 \%$ of all cases for the given network structure.

\subsection{Response to small local changes}

Alterations of the capacities of a single transmission line can strongly impact the global operation of a network already far before the line breaks down. To characterize the relevance of an edge in this respect, we analyze how a local perturbation affects the global steady state in linear response theory. We assume again that every transmission line has the same capacity. We thus consider a small perturbation $\kappa_{i j}$ of the network at a single edge $(a, b)$ such that $K_{i j}^{\prime}=K_{i j}+\kappa_{i j}$ with $\kappa_{a b}=\kappa_{b a}=\kappa$ and $\kappa_{i j}=0$ for all other edges. We thus have

$$
\begin{aligned}
K_{a b}^{\prime} & =K_{a b}+\kappa, \\
K_{i j}^{\prime} & =K_{i j} \quad \text { for } \quad(i j) \neq(a b) .
\end{aligned}
$$

This perturbation induces a small change

$$
\theta_{i} \rightarrow \theta_{i}^{\prime}
$$

of the steady state phases of the network. We thus define the square of the edge susceptibility

$$
\chi_{a b}^{2}:=\lim _{\kappa \rightarrow 0} \frac{\sum_{i}\left|\theta_{i}^{\prime}-\theta_{i}\right|^{2}}{\kappa^{2}} .
$$

as the squared relative change of the steady state relative to the perturbation strength $\kappa$. Here we fix an overall phase shift by imposing the constraint that $\sum_{i} \theta_{i}=0=\sum_{i} \theta_{i}^{\prime}$. To relate the susceptibility of an edge to its load and its redundancy, we calculate the response pattern

$$
\xi_{i}:=\theta_{i}^{\prime}-\theta_{i}
$$

in linear response. 


\section{Power outages and critical transmission lines}

The steady state of the original network is defined via (cf. section 4.2.4)

$$
0=P_{i}+\sum_{j=1}^{N} K_{i j} \sin \left(\theta_{j}-\theta_{i}\right) .
$$

Consequently, the steady state of the perturbed network is

$$
0=P_{i}+\sum_{j} K_{i j}^{\prime} \sin \left(\theta_{j}+\xi_{j}-\theta_{i}-\xi_{i}\right)
$$

For the perturbed network we expand the steady state condition to leading order in $\kappa_{i j}$ and $\xi_{j}:=\theta_{j}^{\prime}-\theta_{j}$ with the result

$$
\begin{aligned}
0 & =P_{i}+\sum_{j} K_{i j}^{\prime} \sin \left(\theta_{j}-\theta_{i}\right)+\sum_{j} K_{i j}^{\prime} \cos \left(\theta_{j}-\theta_{i}\right)\left(\xi_{j}-\xi_{i}\right) \\
& =\underbrace{P_{i}+\sum_{j} K_{i j} \sin \left(\theta_{j}-\theta_{i}\right)}_{=0}+\sum_{j} \kappa_{i j} \sin \left(\theta_{j}-\theta_{i}\right)+\sum_{j} K_{i j} \cos \left(\theta_{j}-\theta_{i}\right)\left(\xi_{j}-\xi_{i}\right)+O\left(\xi^{2}\right) \\
& =\sum_{j=1}^{N} K_{i j} \cos \left(\theta_{j}-\theta_{i}\right)\left(\xi_{j}-\xi_{i}\right)+\sum_{j=1}^{N} \kappa_{i j} \sin \left(\theta_{j}-\theta_{i}\right)
\end{aligned}
$$

for all $i \in\{1, \ldots, N\}$. We can simplify this result using the definition of the power flow $F_{i j}$ (cf. (7.2p) under the assumption of $\left|\theta_{j}-\theta_{i}\right| \leq \pi / 2$. This assumption almost always holds, the only exceptions that may occur are for heavily loaded transmission lines. However, here we are considering transmission lines that carry loads considerably below their maximum transmission capacity. Using the trigonometric identity $\cos ^{2}(x)+\sin ^{2}(x)=1$ yields

$$
\begin{aligned}
K_{i j} \cos \left(\theta_{j}-\theta_{i}\right) & =\sqrt{K_{i j}^{2}-\left(K_{i j} \sin \left(\theta_{j}-\theta_{i}\right)\right)^{2}}, \\
& =\sqrt{K_{i j}^{2}-F_{i j}^{2}} .
\end{aligned}
$$

The expression $\sqrt{K_{i j}^{2}-F_{i j}^{2}}$ can be understood as the residual capacity of each edge, i.e., the free capacity which can be used to react to the perturbation (cf. section 7.4.2). Because the system is in the stability region and far away from being overloaded we have $\left|F_{i j}\right|<K_{i j}$. Equation $7.18 \mathrm{c}$ then reads

$$
0=\sum_{j=1}^{N} \sqrt{K_{i j}^{2}-F_{i j}^{2}}\left(\xi_{j}-\xi_{i}\right)+\kappa \frac{F_{a b}}{K_{a b}} q_{i}
$$


for all $i \in\{1, \ldots, N\}$ with

$$
q_{i}= \begin{cases}1 & \text { for } i=a \\ -1 & \text { for } i=b \\ 0 & \text { else }\end{cases}
$$

With the definition of the residual network $\widetilde{K}_{i j}$ as

$$
\widetilde{K}_{i j}:=\sqrt{K_{i j}^{2}-F_{i j}^{2}}
$$

we thus have

$$
\begin{aligned}
\kappa \frac{F_{a b}}{K_{a b}} q_{i} & =\sum_{j=1}^{N} \widetilde{K}_{i j}\left(\xi_{i}-\xi_{j}\right) \\
& =\sum_{j=1}^{N} \widetilde{K}_{i j} \xi_{i}-\sum_{j=1}^{N} \widetilde{K}_{i j} \xi_{j} .
\end{aligned}
$$

The degree matrix $D$ of a matrix $M$ is defined as the diagonal matrix whose $i$-th diagonal entry, $d_{i i}$, is $\sum_{k} m_{i k}$, the sum of the entries in the $i$-th row of $M$. The matrix $\widetilde{K}$ is symmetric, therefore the first sum of equation $(7.23 \mathrm{~b})$ is equal to the product of the degree matrix of $\widetilde{K}$ and $\vec{\xi}$. The second sum equals the product of $\widetilde{K}$ and $\vec{\xi}$. In a short-hand vectorial notation, we thus find

$$
L(\widetilde{K}) \vec{\xi}=\kappa \frac{F_{a b}}{K_{a b}} \vec{q},
$$

where the matrix $L(\widetilde{K})$ the Laplacian matrix of the residual network $\widetilde{K}_{i j}$. The Laplacian matrix of a matrix is defined as the difference of its degree and adjacency matrix [91].

For the response pattern $\vec{\xi}$ we thus have

$$
\vec{\xi}=\kappa \frac{F_{a b}}{K_{a b}} L^{\dagger}(\widetilde{K}) \vec{q}
$$

with $L^{\dagger}(\widetilde{K})[92$ as the so called generalized inverse of $L(\widetilde{K})$. The eigenvalues of a Laplacian matrix are $0=a_{1}<a_{2}<\cdots a_{N}$ with corresponding eigenvectors $\vec{v}_{n}$ [93. The eigenvalues of the generalized inversed of the Laplacian matrix are $1 / a_{n}$ with $1 / a_{1}$ excluded [92]. The eigenvectors are the same with the exclusion of $\vec{v}_{1}$ [92]. We thus have

$$
\vec{\xi}=\kappa \frac{F_{a b}}{K_{a b}} \sum_{n=2}^{N} \frac{1}{a_{n}}\left|\vec{v}_{n} \cdot \vec{q}\right| .
$$

A deeper understanding of the susceptibility of each edge can be obtained by solving equation 7.26 for $\vec{\xi}$ and substituting the result into the definition 7.14. Then the edge 


\section{Power outages and critical transmission lines}

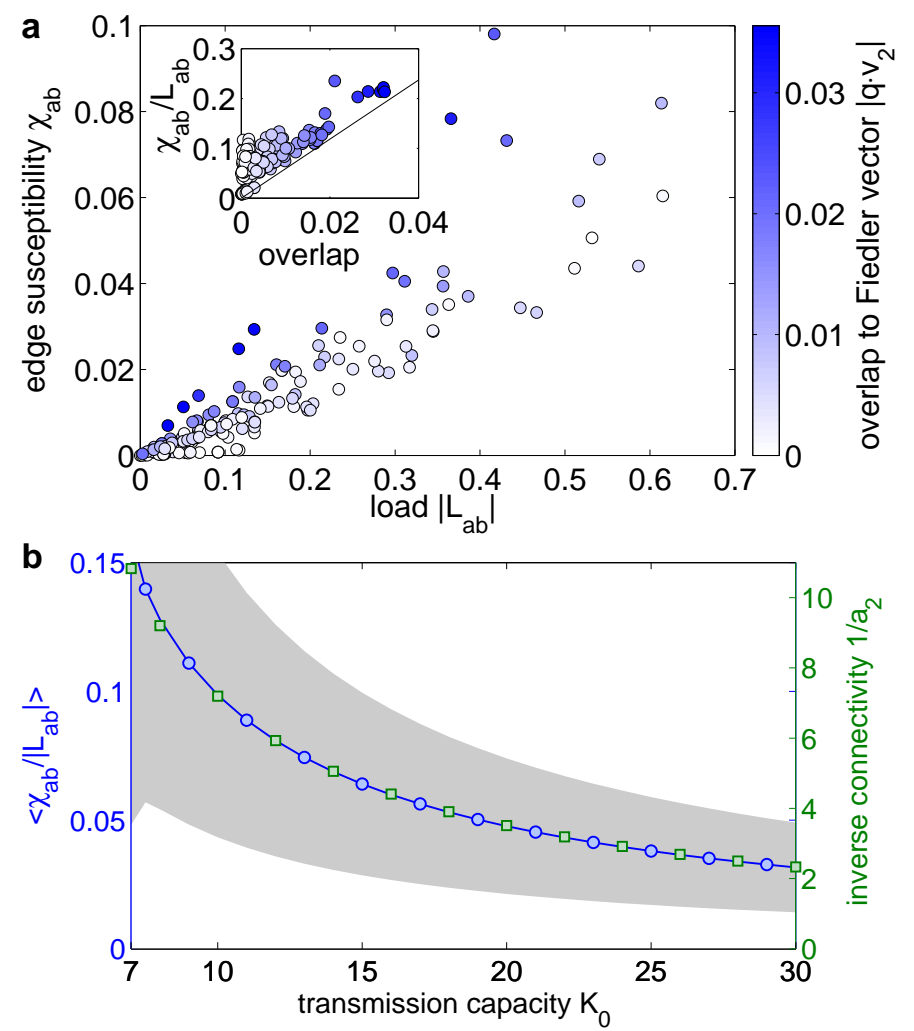

Figure 7.7: The edge susceptibility $\chi_{a b}$ is determined by the load and the algebraic connectivity. (a) For a given network, the susceptibility is approximately proportional to the load of the edge $L_{a b}$. It is increased if the edge $(a, b)$ couples two weakly connected components of the residual capacity graph $\tilde{K}$, indicated by a large overlap with the Fiedler vector $\left|\vec{q} \cdot \vec{v}_{2}\right|$ (shown as a color code and in the inset). (b) On a global scale, the average susceptibility is proportional to the inverse algebraic connectivity $1 / a_{2}$. The plot shows $1 / a_{2}(\square)$ and the ratio $\chi_{a b} /\left|L_{a b}\right|$ averaged over all edges (o) as a function of the transmission capacity $K_{0}$. The shading shows the standard deviation of $\chi_{a b} /\left|L_{a b}\right|$. The network is as in Fig. 7.1

susceptibility is given by

$$
\chi_{a b}^{2}=L_{a b}^{2} \sum_{n=2}^{N} \frac{1}{a_{n}^{2}}\left|\vec{v}_{n} \cdot \vec{q}\right|^{2} .
$$

This expression shows three important results:

(1) The edge susceptibility generally scales with the load of the edge $L_{a b}=F_{a b}^{(0)} / K_{a b}$.

(2) The prefactors $1 / a_{n}^{2}$ decrease with $n$. In particular for a heavily loaded network the factor $1 / a_{2}^{2}$ is very large compared to the others and the term $n=2$ dominates the sum. Then the susceptibility of all edges in the network scales inversely with the eigenvalue $a_{2}$, which measures the algebraic connectivity [94 96]. Hence, the susceptibility is large if the network defined by the residual capacities $\sqrt{K_{i j}^{2}-F_{i j}^{2}}$ is weakly connected. This is illustrated in Figure 7.7(b). 
2) The prefactors $1 / a_{n}^{2}$ decrease with $n$. In particular for a heavily loaded network the factor $1 / a_{2}^{2}$ is very large compared to the others and the term $n=2$ dominates the sum. Then the susceptibility of all edges in the network scales inversely with the eigenvalue $a_{2}$, which measures the algebraic connectivity $94-96]$. Hence, the susceptibility is large if the network defined by the residual capacities $\sqrt{K_{i j}^{2}-F_{i j}^{2}}$ is has a low algebraic connectivity. This is illustrated in Figure 7.7(b).

(3) For a heavily loaded network, the edge susceptibility scales with the overlap $\left|\vec{v}_{2} \cdot \vec{q}\right|$, where $\vec{v}_{2}$ is the so-called Fiedler vector $[97$. This overlap can be interpreted as a measure of the local algebraic connectivity of the nodes $a$ and $b$. To see this note that the Fiedler vector can be used to partition a graph into two weakly connected parts 95,96 . The overlap with the vector $\vec{q}$ is largest if the nodes $a$ and $b$ are in two different weakly connected parts of the grid. To make this reasoning most clearly, we consider the limiting case $a_{2} \rightarrow 0$, i.e., the residual network defined by $\sqrt{K_{i j}^{2}-F_{i j}^{2}}$ becomes disconnected into two components. The Fiedler vector then reads $\vec{v}_{2} \sim(1, \ldots, 1,-1, \ldots,-1)$, with entries +1 for the nodes in the first component and -1 for the nodes in the second component [98, 99]. The overlap is then given by (cf. (equations 7.21) )

$$
\left|\vec{v}_{2} \cdot \vec{q}\right|= \begin{cases}0 & \text { if } a \text { and } b \text { are in the same fragment } \\ \frac{2}{\sqrt{N}} & \text { if } a \text { and } b \text { are in different fragments. }\end{cases}
$$

Therefore the susceptibility tends to infinity as $a_{2} \rightarrow 0$ if the edge $(a, b)$ links the two fragments, whereas it remains finite otherwise. This is illustrated in Figure 7.7 (a).

The numerical data shown in Figure 7.7 clearly confirm these findings. The susceptibility of the individual edges scales with the load $L_{a b}$ and the overlap to the Fiedler vector $\left|\vec{v}_{2} \cdot \vec{q}\right|$, whereas the average susceptibility is proportional to the inverse connectivity $1 / a_{2}$.

\subsection{Discussion}

In summary, we have characterized critical infrastructures of complex power grids. We linked the graph theoretical measure of edge connectivity with dynamical features of complex power grids. We thereby demonstrated that a grid's response to both small damages and complete breakdowns of single edges, can be predicted with high accuracy from topological and load properties of the grid's original unperturbed state that are a priori accessible. The breakdown of a single edge is very likely to cause a global desynchronization of the power grid if there is at most one redundant path with low redundant capacity to take over the flow of the failing edge. In contrast, the load of the edge alone does not provide a reliable indicator for the criticality of an edge. To quantify the effects of small damages, we have defined the edge susceptibility which measures the change of the global steady state caused by a small local perturbation. The edge susceptibility is essentially determined by the load and the algebraic connectivity of the network. 
Our work applies especially to situations of extreme loads where the power grid becomes vulnerable to large-scale outages. These situations are expected to become much more frequent in future sustainable power grids. The criteria identified may thus find valuable applications for predicting, and potentially preventing large scale outages in real world networks. In particular their a priori nature permits ad-hoc analysis of grid operation in real-time, also because they avoid simulations of outages that may be highly computationally demanding and time-critical. They may thus improve existing stability regulations for power grid operation [23 and moreover be used for a rapid assessment of grid structures in network planning and optimization. 


\section{Conclusions}

In this thesis, we studied the synchronization properties in models of power grids and how they relate to stable operation thereof. In the class of models considered, every generator and consumer of a grid is represented by a second order oscillator (nodes) and the different oscillators are coupled by transmission lines (edges). The collective dynamics of the power grid therefore depends on the interactions of the oscillators. We focused on two challenges for power grids. The first is to determine the influence of decentralization of power generation on the operation of power grids. The second is to determine which transmission lines are the most crucial for the operation of the grid, i.e., can cause power outages in case of their failure.

To study the large-scale dynamic behavior of power grids we used the oscillator power grid model derived by Filatrella et al. [29]. This model allows to simulate the dynamics of power grids with a set of parameters, which model the power output of real power sources. To model the largely complex power grid a number of simplifications had to be implemented. They are, in short, the reduction of the elements of the power grid to three different elements: generators, consumers and transmission lines. Every consumer and generator is represented by a synchronous machine. Ohmic losses in transmission lines are discarded and we chose a homogeneous set of parameters. Although these simplifications are well reasoned, real power grids possess of course none of these simplifications. Still, despite all these shortcomings, the oscillator power grid model is a powerful tool to analyze the behavior of power grids. It can represent one of the most important features of real power grids, that is the coexistence of a stable and an unstable state. For instance, only this feature allows studies about the reaction of the power grid to perturbations of power consumption, because it is a priori not clear if the grid can go back to a steady phaselocked state after the perturbation is removed or not. Thus, the model is an appropriate tool to analyze collective power grid dynamics. We studied the collective dynamics of power grids for four different topology classes, three general topologies, random, small-world and quasi-regular, and the special topology of the British high-voltage power grid.

In the first part of the thesis (Chapter 4 and 5 ) we presented the model and analyzed the special case of a power grid consisting of only two elements, a generator and a consumer. This special case is analytically tractable. We presented a detailed bifurcation study of this power grid. We showed that already this simple system contains features that are evidently also present in real power grids, mainly the coexistence of a stable and an unstable state of power grid operation. Therefore the collective dynamics of the model crucially depend on the initial conditions, they can either converge to stable fixed point or the limit cycle. 


\section{Conclusions}

Thus, the model allows insights into the behavior of more complex topologies.

In the second part (Chapter 6) we studied the process of decentralization of power generation within the oscillator power grid model - an ongoing process in real world power grids. We analyzed different aspects of this process. First, we showed that for decentralized power grids the capability of reaching a steady phase-locked state of power grid operation can be achieved with lower transmission line capacities than for centralized power grids. Second, we showed that the synchronization time, i.e., the time the grid needs to actually reach the steady phase-locked state, does not increase by decentralizing the grid. Third, we demonstrated that the dynamical stability, i.e., the capability of the power grid to maintain stable operation in case of uneven power production and consumption, is negatively affected by decentralization. Finally, we showed that the structural stability, i.e., the capability to maintain stable operation for transmission line failures, generally increases for decentralized power grids. Thus already the decentralization of the grid, which is only one aspect of the so-called Energiewende, has complex, oppositional effects on the structural stability of the grid.

The third part (Chapter 7) of the thesis is devoted to a more detailed analysis of the structural stability. We showed that the load of a transmission line has almost no influence on the global stability in case of its failure. Instead, redundancy is the key concept to detect critical transmission lines in power grids. A rough prediction of grid stability is already provided by the local edge connectivity, which essentially measures the number of different alternative paths for the power flow of a transmission line. A reliable prediction is feasible by comparing the load of the failing edge with the redundant capacity of the grid. Intuitively, the redundant capacity is a measure of how much more free transmission capacity is left for the rerouting of the power flow of the failed transmission line. It is calculated on the basis of the grid structure and the operation before the failure using graph-theoretic algorithms and requires neither dynamical simulations nor knowledge of the details of the supply network model. Furthermore, we analytically derived the susceptibility of the power grid in response to a small change in the capacity of one transmission line of the power grid. We confirmed that the local algebraic connectivity of the network of the residual capacities is decisive for the susceptibility of the grid to small damages of single transmission lines. To our knowledge, the concept of the residual capacities and the derivation of the edge susceptibility is fundamentally new and previously unknown in the literature.

Our findings regarding the structural stability and the reasons for the criticality of transmission lines are examples of finding fundamental principles. Although real power grids possess of course a more inhomogeneous parameter setting than our model, we expect that their principal behavior regarding the structural stability is comparable. Therefore our findings can be viewed as fundamental predictions regarding the behavior of real power grids. Our results regarding decentralization of power generation and the structural stability are the prime examples. They can serve as a guideline for the development of a protocol that determines power grid stability: Our model points towards the properties of 
transmission lines that have to be checked in real power grids to predict their criticality for power grid operations prior to potential breakdowns, such that countermeasures can be executed in time. Our results can also be used to identify permanently critical transmission lines, which have low redundant capacities and thus serve as a guideline to identify necessities for the building of new redundant transmission lines.

Extensions of the work presented in this thesis open further novel perspectives to interesting questions of power grid operation. For instance, the concept of residual capacities may be used as a guideline to analyze possible counteracting strategies for the failures of power sources. Which other power sources should optimally increase their power output as a compensation? Furthermore, it will be necessary to implement storage facilities into a power grid consisting of mainly renewable power sources [12]. This raises the related question of how they should operate in the event of failures of power sources.

It will be an interesting future task to confirm our results regarding the properties that determine the criticality of transmission lines with data of real power grids. Although the N-1 safety criterion [24] (that the grid should always be capable of maintaining its stable state despite the breakdown of any given transmission line), is already established for real power grids, violations of this criterion occur from time to time. To show that these events may be related to a state of a weakly connected graph of residual capacities prior to the breakdown line should be an aim of future research.

Maintaining stable operation was always a challenging problem for power grids. Current sophisticated strategies have been developed over the last few decades. With the ongoing changes in power generation with an increasing fraction of renewable sources and thus more fluctuations in the power output, it is a priori not clear if these strategies will hold. For instance an enormous challenge for the development of power grids is that often wind energy sources are built predominantly at the seaside such that energy is generated far away from consumers. The insights and methods presented here may further our understanding of the collective dynamics of today's power grids as well as help investigating different scenarios for upgrading the grid. Here we have contributed to the development of new strategies of maintaining stable operation for future power grids. 



\section{Acknowledgments}

An dieser Stelle möchte ich allen Personen danken, die mich während meiner Zeit als Doktorand unterstützt haben. Zuallererst möchte ich mich bei Marc Timme bedanken, der mir die Möglichkeit zur Anfertigung dieser Arbeit gegeben hat. Vielen Dank auch für die hervorragende Unterstützung.

Weiterhin möchte ich mich ganz besonders bei Dirk Witthaut bedanken. Die viele hilfreichen Diskussionen und Anregungen über die gesamte Zeit der Arbeit hinweg waren von unschätzbarer Wichtigkeit. Ausserdem möchte ich Andreas Sorge danken, insbesondere für die vielen wertvollen Ideen gerade zu Beginn der Arbeit.

My special thanks go to all members of the Network Dynamics Group. The atmosphere in the group was always wonderful for both work-related discussions as well as for trips away from the offices. We spent so much time together for retreats, barbecues and much more. It was always a lot of fun. I especially enjoyed our little breaks for playing table tennis in the afternoon. Many thanks to Jose Casadiego, Fabio Schittler Neves, Hinrich Arnoldt and Gunter Weber for all these funny little matches. Mein ganz besonderer Dank gilt Hinrich Arnoldt, der über viele Jahre ein Büro mit mir teilen musste und mich trotzdem täglich mit einer Tasse Tee versorgt hat. Vielen Dank! Danken möchte ich auch Gunter Weber, Harold Gutch und Sven Jahnke, die mir in den letzten sehr arbeitsintensiven Monaten eine grosse Stütze waren. Ich werde unsere "Wochenendkleingruppe" und die abendlichen Pizzarunden vermissen.

Vielen Dank auch noch einmal an Harold Gutch und Sven Jahnke, für die enorm hilfreiche technische Unterstützung, wann immer Probleme mit den Rechnern aufgetaucht sind. Vielen Dank auch an Barbara Kutz für ihre Unterstützung was sämtliche Verwaltungsvorgänge angeht.

Für viele großartige gemeinsam verbrachte Stunden und anregende Gespräche abseits der Wissenschaft möchte ich Ulf Seemann und Gunter Weber danken. Bei Jan Schlüter möchte ich mich für seine Idee zur Ausrichtung des Science Volleyballcups 2012/13 zwischen den Göttinger MPIs bedanken. Bei der Organisation mitzuhelfen war sehr spannend. Stellvertretend für den gesamten TTC Göttingen möchte ich mich bei Jörg Vogl bedanken, mit dem ich über viele Jahre in einer Mannschaft gespielt habe. Es hat mir immer enorm viel Spaß gemacht und war ein toller Ausgleich zum wissenschaftlichen Arbeiten.

Zum Schluss möchte ich meiner geliebten Mutter Gedenken, die das Enstehen dieser Arbeit leider nicht mehr erleben konnte. 


\title{
Martin Rohden
}

\author{
Personal data \\ Nationality German \\ Date of birth August 25th, 1981 \\ Place of birth Uelzen \\ Marital status Unmarried \\ Mother tongue German
}

\section{University Education}

10/2002-11/2008 Undergraduate studies in Physics.

Georg-August-Universität Göttingen

10/2004 Vordiplom in Physics.

Georg-August-Universität Göttingen

02/2006-06/2006 Study abroad, Erasmus semester at the Elte University in Budapest.

10/2007-09/2008 Diplomarbeit, Independent Component Analysis for spiking neurons, Institute for Nonlinear Dynamics, Max Planck Institute for Dynamics and SelfOrganization.

11/2008 Diplom in Physics.

11/2009-01/2014 PhD student, Network Dynamics Group, Max-Planck Institute for Dynamics and Self-Organization, University of Göttingen, GGNB program physics of biological and complex systems.

\section{Work Experience}

02/2009-10/2009 Scientific Assistant, Network Dynamics Group, Max-Planck Institute for Dynamics and Self-Organization.

\section{Awards}

08/2010-07/2011 GGNB Stipend, Göttingen Graduate School for Neuroscience, Biophysics.

\section{Publications}

Research article Debsankha Manik, Dirk Witthaut, Benjamin Schäfer, Moritz Matthiae, Andreas Sorge, Martin Rohden, Eleni Katifori and Marc Timme, Supply networks: Instabilities without overload, Eur. Phys. J. ST., 223, pp. 2527, 2014.

Research article Martin Rohden, Andreas Sorge, Dirk Witthaut and Marc Timme, Impact of network topology on synchrony of oscillatory power grids, Chaos 24, 013123 , 2014. 
Research article Martin Rohden, Andreas Sorge, Marc Timme and Dirk Witthaut, Self-Organized Synchronization in Decentralized Power Grids, Phys. Rev. Let. 109, 064101, 2012.

School Education

1994-2001 Herzog-Ernst-Gymnasium, Uelzen.

07/2001 Abitur.

Civilian Service

08/2001-05/2002 Kirchengemeinde St. Marien, Uelzen. 



\section{Bibliography}

[1] E. Marris. "Energy: Upgrading the grid". In: Nature 454 (2008), p. 570.

[2] J. A. Turner. "A Realizable Renewable Energy Future". In: Science 285 (1999), p. 687.

[3] Intergovernmental Panel On Climate Change. "Climate change 2007: the physical science basis". In: Agenda 6.07 (2007).

[4] Bundesministerium für Umwelt, Naturschutz und Reaktorsicherheit. Langfristszenarien und Strategien für den Ausbau der erneuerbaren Energien in Deutschland bei Berücksichtigung der Entwicklung in Europa und global. http: / /www . bmu.de / files/pdfs / allgemein/application/pdf / leitstudie2010_bf .pdf (retrieved 20/2/2013). 2010.

[5] Bundesministerium für Umwelt, Naturschutz und Reaktorsicherheit. Entwicklung der spezifischen Kohlendioxid-Emissionen des deutschen Strommix. Umweltbundesamt, 2007.

[6] Bundesministerium für Umwelt, Naturschutz und Reaktorsicherheit. Zeitreihen zur Entwicklung der erneuerbaren Energien in Deutschland. Umweltbundesamt, 2013.

[7] Bundesministerium für Umwelt, Naturschutz und Reaktorsicherheit. Erneuerbare Energien in Zahlen-nationale und internationale Entwicklung. Umweltbundesamt, 2013.

[8] Europäisches Parlament und der Rat der europäischen Union. "Richtlinie 96/92/EG des Europäischen Parlaments und des Rates vom 19. Dezember 1996 betreffend gemeinsame Vorschriften für den Elektrizitätsbinnenmarkt". In: Amtsblatt Nr. L 27 (1996), p. 0020.

[9] Deutscher Bundestag. "Gesetz über den Vorrang Erneuerbarer Energien (Erneuerbare-Energien-Gesetz, EEG)". In: Bundesgesetzblatt Teil I Nr. 13 (2000).

[10] P. Milan, M. Wächter, and J. Peinke. "Turbulent Character of Wind Energy". In: Physical Review Letters 110.13 (2013).

[11] D. Heide et al. "Seasonal optimal mix of wind and solar power in a future, highly renewable Europe". In: Renewable Energy 35 (2010), p. 2483.

[12] D. Heide et al. "Reduced storage and balancing needs in a fully renewable European power system with excess wind and solar power generation". In: Renewable Energy 36 (2011), p. 2515. 
[13] J. C. Smith et al. "Utility Wind Integration and Operating Impact State of the Art". In: IEEE Transactions on Power Systems 22.3 (2007), pp. 900-908.

[14] T. Ackermann, G. Andersson, and L. Söder. "Distributed generation: a definition". In: Electric Power Systems Research 57 (2001), pp. 195 -204.

[15] T. Ackermann. Wind Power in Power Systems. Wiley, 2012.

[16] F. Trieb. "Strom aus der Wüste. DLR-Studien zum Projekt Desertec". In: Physik in unserer Zeit 42.2 (2011), pp. 84-91.

[17] A.-M. Borbely and J. F. Kreider. Distributed Generation: The Power Paradigm for the New Millennium (Mechanical and Aerospace Engineering Series). CRC Press, 2001.

[18] Bundesnetzagentur. Netzentwicklungsplan 2012. http : / / www . netzentwicklungsplan.de (retrieved 20/2/2013). 2012.

[19] M. Rohden et al. "Self-organized synchronization in decentralized power grids". In: Phys. Rev. Lett. 109:064101 (2012).

[20] A. E. Motter et al. "Spontaneous synchrony in power-grid networks". In: Nature Physics 9 (2013), p. 191.

[21] S. Lozano, L. Buzna, and A. Díaz-Guilera. "Role of network topology in the synchronization of power systems". In: The European Physical Journal B - Condensed Matter and Complex Systems 85 (2012), p. 231.

[22] K. Heuck, K.-D. Dettmann, and D. Schulz. Elektrische Energieversorgung. Wiesbaden: Vieweg+Teubner, 2010.

[23] European Network of Transmission System Operators for Electricity. Operation Handbook. 2004.

[24] P. Kundur. Power System Stability and Control. New York: McGraw-Hill, 1994.

[25] J. Machowski, J. Bialek, and J. Bumby. Power system dynamics, stability and control. New York: John Wiley \& Sons, 2008.

[26] T. Petermann et al. Was bei einem Blackout geschieht. Edition Sigma, 2011.

[27] Bundesnetzagentur. Bericht über die Systemstörung im deutschen und europäischen Verbundsystem am 4. November 2006. http://www . bundesnetzagentur. de/SharedDocs / Downloads / DE/BNetzA / Sachgebiete / Energie/Sonderthemen/ Stromausfall4Nov06/BerichtId9007pdf .pdf (retrieved 20/2/2013). 2007.

[28] W. Kröger and E. Zio. Vulnerable Systems. Springer, 2011.

[29] G. Filatrella, A. H. Nielsen, and N. F. Pedersen. "Analysis of a power grid using a Kuramoto-like model". In: Eur. Phys. J. B 61.4 (2008), p. 485.

[30] Y. Kuramoto. Chemical Oscillations, Waves, and Turbulence. Berlin: Springer, 1984.

[31] European Network of Transmission System Operators for Electricity. http://www. entsoe.eu/. 
[32] Bundesverband der Energie- und Wasserwirtschaft. Deutsches Stromnetz ist 1,78 Millionen Kilometer lang. 2010.

[33] G. R. Kirchhoff. "Ueber den Durchgang eines elektrischen Stromes durch eine Ebene, insbesondere durch eine kreisförmige". In: Annalen der Physik und Chemie 140.4 (1845), pp. 497-514.

[34] D. Heide, M. Schäfer, and M. Greiner. "Robustness of networks against fluctuationinduced cascading failures". In: Phys. Rev. E 77 (2008), p. 056103.

[35] A. E. Motter and Y.-C. Lai. "Cascade-based attacks on complex networks". In: Phys. Rev. E 66 (2002), p. 065102.

[36] M. Schäfer, J. Scholz, and M. Greiner. "Proactive Robustness Control of Heterogeneously Loaded Networks". In: Phys. Rev. Lett. 96 (2006), p. 108701.

[37] I. Simonsen et al. "Transient Dynamics Increasing Network Vulnerability to Cascading Failures". In: Phys. Rev. Lett. 100 (2008), p. 218701.

[38] J. Nagler, A. Levina, and M. Timme. "Impact of single links in competitive percolation". In: Nature Physics 7 (2011), p. 265.

[39] Siemens. Power System Simulator for Engineering (PSS/E). http://www . energy. siemens.com

[40] C. Gerthsen. Gerthsen Physik (Springer-Lehrbuch) (German Edition). Springer, 2010 .

[41] T. Cutsem and C. Vournas. Voltage Stability of Electric Power Systems (Power Electronics and Power Systems). Springer, 2007.

[42] A. Kronenberger. Elektrischer Generator. 2007.

[43] M. Matthiae. Stabilität oszillatorischer Stromnetze gegen stochastische und periodische Leistungsfluktuationen. Bachelor thesis, Uni Göttingen, 2012.

[44] M. Weixelbraun. Dynamische Simulation eines 110-kv-Netzes im Inselnetzbetrieb. Diplomarbeit, TU Graz, 2007.

[45] A. T. Winfree. "Biological rhythms and the behavior of populations of coupled oscillators". In: Journal of Theoretical Biology 16.1 (1967), pp. 15-42.

[46] J. A. Acebrón et al. "The Kuramoto model: A simple paradigm for synchronization phenomena". In: Rev. Mod. Phys. 77 (2005), p. 137.

[47] B. Ermentrout. "An adaptive model for synchrony in the firefly pteroptyx malaccae". In: Journal of Mathematical Biology 29.6 (1991), p. 571.

[48] S. H. Strogatz et al. "Crowd synchrony on the Millennium Bridge". In: Nature 438.7064 (2005), p. 43.

[49] S. H. Strogatz. Sync: How Order Emerges From Chaos In the Universe, Nature, and Daily Life. Hyperion, 2004. 
[50] A. Bergen and D. Hill. "A Structure Preserving Model for Power System Stability Analysis". In: IEEE Transactions on Power Apparatus and Systems PAS-100.1 (1981), pp. 25-35.

[51] F. Dörfler and F. Bullo. "Synchronization and Transient Stability in Power Networks and Nonuniform Kuramoto Oscillators". In: SIAM Journal on Control and Optimization 50.3 (2012), pp. 1616-1642.

[52] L. Buzna, S. Lozano, and A. Díaz-Guilera. "Synchronization in symmetric bipolar population networks". In: Phys. Rev. E 80 (2009), p. 066120.

[53] D. F. Simpson-Porco John W. and F. Bullo. "Synchronization and power sharing for droop-controlled inverters in islanded microgrids". In: Automatica 49.9 (2013), pp. 2603-2611.

[54] D. Subbarao and a. B. S. R. Uma. "Self-organization on a power system". In: IEEE Power Engineering Review 21 (12) (2001), pp. 59-62.

[55] D. Hill and G. Chen. "Power systems as dynamic networks". In: Proceedings of the 2006 IEEE International Symposium on Circuits and Systems. 2006, p. 725.

[56] V. Fioriti et al. Stability of a Distributed Generation Network Using the Kuramoto Models. Springer-Verlag, 2009, pp. 14-23.

[57] K. Schmietendorf et al. "Self-Organized Synchronization and Voltage Stability in Networks of Synchronous Machines". In: (2013).

[58] M. Rohden et al. "Impact of network topology on synchrony of oscillatory power grids". In: Chaos 24: 013123 (2014).

[59] S. H. Strogatz. Nonlinear Dynamics And Chaos: With Applications To Physics, Biology, Chemistry, And Engineering (Studies in Nonlinearity). Westview Press, 2001.

[60] H. Risken. The Fokker-Planck Equation. Berlin Heidelberg: Springer, 1996.

[61] International Energy Agency. Distributed Generation in Liberalised Electricity Markets. 2002.

[62] G. Pepermans et al. "Distributed generation: definition, benefits and issues". In: Energy Policy 33.6 (2005), p. 787.

[63] N. Strachan, H. Zerriffi, and H. Dowlatabadi. System Implications of Distributed Generation. Springer-Verlag, 2003, pp. 39-75.

[64] A. Gheorghe et al. Critical Infrastructures at Risk : Securing the European Electric Power System (Topics in Safety, Risk, Reliability and Quality). Springer, 2006.

[65] P. Erdős and R. A. "On random graphs I." In: Publ. Math. Debrecen 6 (1959), pp. 290-297.

[66] P. Erdős and A. Rényi. "On the Evolution of Random Graphs". In: Publication of the Mathematical Institute of the Hungarian Academy of Sciences. 1960, pp. 17-61. 
[67] D. Watts and S. H. Strogatz. "Collective dynamics of 'small-world' networks". In: Nature 393 (1998), p. 440.

[68] S. H. Strogatz. "From Kuramoto to Crawford: Exploring the onset of synchronization in populations of coupled oscillators". In: Physica D: Nonlinear Phenomena 143 (2000), p. 1.

[69] N. E. J. Newman. "The Structure and Function of Complex Networks". In: SIAM Review 45 (2003), p. 167.

[70] F. Blaabjerg et al. "Overview of Control and Grid Synchronization for Distributed Power Generation Systems". In: IEEE Transactions on Industrial Electronics 53.5 (2006), pp. $1398-1409$.

[71] M. Timme, F. Wolf, and T. Geisel. "Topological Speed Limits to Network Synchronization". In: Physical Review Letters 92.7 (2004).

[72] C. Grabow et al. "Do small worlds synchronize fastest?" In: EPL (Europhysics Letters) 90.4 (2010), p. 48002.

[73] C. Grabow, S. Grosskinsky, and M. Timme. "Speed of complex network synchronization". In: The European Physical Journal B 84.4 (2011), pp. 613-626.

[74] P. Milan, M. Wächter, and J. Peinke. "Stochastic modeling of wind power production". In: Proceedings of EWEA (2011).

[75] D. Energie-Agentur. "Energiewirtschaftliche Planung für die Netzintegration von Windenergie in Deutschland an Land und Offshore bis zum Jahr 2020". In: Konsortium DEWI/E. ON Netz/EWI/RWE Transportnetz Strom/VE Transmission, Endbericht, Köln (2005).

[76] R. Albert, H. Jeong, and A. Barabási. "Error and attack tolerance of complex networks". In: Nature 406 (2000), p. 378.

[77] R. Cohen et al. "Breakdown of the Internet under Intentional Attack". In: Phys. Rev. Lett. 86 (2001), p. 3682.

[78] A. E. Motter. "Cascade Control and Defense in Complex Networks". In: Phys. Rev. Lett. 93 (2004), p. 098701.

[79] R. Albert, I. Albert, and G. L. Nakarado. "Structural vulnerability of the North American power grid". In: Phys. Rev. E 69 (2004), p. 025103.

[80] P. Crucitti, V. Latora, and M. Marchiori. "Model for cascading failures in complex networks". In: Phys. Rev. E 69 (2004), p. 045104.

[81] L. K. Gallos et al. "Stability and Topology of Scale-Free Networks under Attack and Defense Strategies". In: Phys. Rev. Lett. 94 (2005), p. 188701.

[82] S. V. Buldyrev et al. "Catastrophic cascade of failures in interdependent networks". In: Nature 464.7291 (2010), p. 1025.

[83] C. M. Schneider et al. "Mitigation of malicious attacks on networks". In: Proceedings of the National Academy of Sciences 108 (2011), p. 3838. 
[84] D. Witthaut and M. Timme. "Braess paradox in oscillator networks, desynchronization and power outage". In: New J. Phys. 14, 083036 (2012).

[85] P. Pourbeik, P. Kundur, and C. Taylor. "The anatomy of a power grid blackout Root causes and dynamics of recent major blackouts". In: IEEE Power and Energy Magazine 4.5 (2006), pp. 22-29.

[86] P. J. Menck et al. "How basin stability complements the linear-stability paradigm". In: Nature Physics 9 (2013), p. 89.

[87] D. Jungnickel. Graphs, Networks and Algorithms. Heidelberg Berlin: Springer, 2007.

[88] North American Electric Reliability Corporation. Electricity Technology Roadmap. 2003.

[89] Electric Power Research Institute. Long-Term Reliability Assessment. 2011.

[90] T. Fawcett. ROC Graphs: Notes and Practical Considerations for Data Mining Researchers. 2003.

[91] R. Merris. "Laplacian matrices of graphs: a survey". In: Linear Algebra and its Applications 197 - 198 (1994), pp. $143-176$.

[92] I. Gutman and W. Xiao. "Generalized inverse of the Laplacian matrix and some applications". In: Bulletin: Classe des sciences mathematiques et natturalles 129.29 (2004), pp. 15-23.

[93] R. Merris. "Laplacian graph eigenvectors". In: Linear Algebra and its Applications 278 (1998), pp. $221-236$.

[94] M. Fiedler. "Algebraic connectivity of graphs". In: Czechoslovak Mathematical Journal 23.2 (1973), pp. 298-305.

[95] M. Newman. Networks - An introduction. Oxford: Oxford University Press, 2010.

[96] S. Fortunato. "Community detection in graphs". In: Physics Reports 486.3 (2010), pp. $75-174$.

[97] M. Fiedler. Combinatorics and graph theory (Banach Center publications). PWNPolish Scientific Publishers, 1989.

[98] M. Fiedler. "A property of eigenvectors of nonnegative symmetric matrices and its application to graph theory." English. In: Czech. Math. J. 25 (1975), pp. 619-633.

[99] C. Ding, X. He, and H. Zha. "A spectral method to separate disconnected and nearlydisconnected Web graph component". In: ACM Press, 2001, pp. 275-280. 


\section{List of Figures}

$4.1 \quad$ Schematic diagram of a three-phase synchronous generator . . . . . . . . . 19

4.2 Scheme of a synchronous machine . . . . . . . . . . . . . . . . . . . . 23

$5.1 \quad$ Stability phase diagram in parameter space . . . . . . . . . . . . . . . 35

$\begin{array}{lll}5.2 & \text { Dynamics of an elementary network with one generator and one consumer } \quad 36\end{array}$

5.3 Tilted washboard potential . . . . . . . . . . . . . . . . . . . . . . . . .

5.4 Imbalanced power production and consumption . . . . . . . . . . . . . . . . . 40

5.5 Adaptation principles . . . . . . . . . . . . . . . . . . . 41

6.1 Cartoons of different network topologies f . . . . . . . . . . . . . . . . . . 47

$6.2 \quad$ Decentralization process of the British power grid . . . . . . . . . . . . . . 48

6.3 Motif networks $\ldots \ldots \ldots \ldots \ldots \ldots \ldots$

6.4 Phase dynamics of a quasi-regular power grid . . . . . . . . . . . . . . . . . . . . . 52

6.5 Synchronization transition for a regular network . . . . . . . . . . . . . 53

6.6 Synchronization transition for different fractions of decentralized energy sources for model networks . . . . . . . . . . . . . . . . 54

6.7 Synchronization transition to self-organized phase-locking in the British

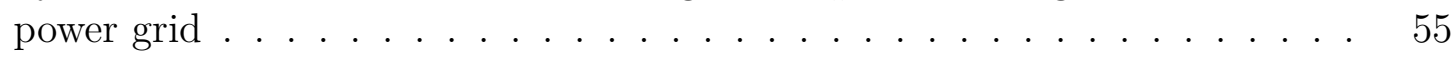

6.8 Synchronization transition for different fractions of decentralized energy sources for the British power grid . . . . . . . . . . . . . . 56

6.9 Synchronization time . . . . . . . . . . . . . . . . . . . . 58

6.10 Effects of weak and strong perturbations . . . . . . . . . . . . . . . 60

6.11 Robustness of three different topology classes . . . . . . . . . . . . . . . . . 61

6.12 Robustness of the British power grid against perturbations in power demand 62

6.13 Structural stability of the British power grid . . . . . . . . . . . . . 63

$7.1 \quad$ Breakdowns of neighboring transmission lines with similar loads . . . . . . 66

7.2 Change of network flows after the removal of one transmission line during

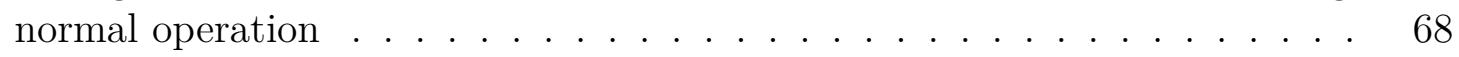

7.3 Network motifs and the definition of local edge connectivity . . . . . . . . 69

7.4 Load and power flow of stable and critical edges . . . . . . . . . . . . . . . . . 71

7.5 Local edge connectivity of stable and critical edges . . . . . . . . . . . . . 72

7.6 Receiver operating characteristics of different classifier systems for critical

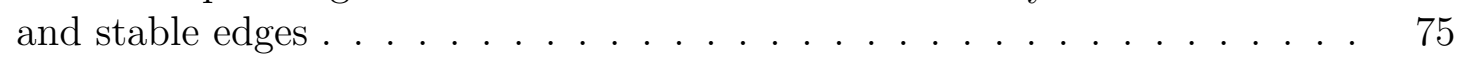

7.7 The edge susceptibility $\chi_{a b} \ldots \ldots \ldots \ldots \ldots$ 